A Study of Polarization in Hyperon Production

Processes

A THESIS

SUBMITTED TO THE FACULTY OF THE GRADUATE SCHOOL OF THE UNIVERSITY OF MINNESOTA

BY

(C) David McDill Woods 1995

David McDill Woods

IN PARTIAL FULFILLMENT OF THE REQUIREMENTS

FOR THE DEGREE OF

DOCTOR OF PHILOSOPHY 


\section{A Study of Polarization in Hyperon Production \\ Processes}

\author{
by David McDill Woods
}

Under the supervision of Professor Kenneth J. Heller

\section{ABSTRACT}

The polarization of $\Xi^{-}$and $\Omega^{-}$hyperons produced from both polarized and unpolarized neutral particle beams has been studied. The unpolarized neutral beam production studies are the first measurements made using this production technique. The neutral beam consisted of neutrons, $\Lambda^{0} \mathrm{~s}, \Xi^{0} \mathrm{~s}, K^{0} \mathrm{~s}$, and photons. No polarization was observed in the sample of $1.4 \times 10^{7} \Xi^{-} \mathrm{s}$ produced by an unpolarized neutral beam. For $\Omega^{-}$s produced by an unpolarized neutral beam, a sample of $1.7 \times 10^{5}$ events with an average momentum of $394 . \mathrm{GeV} / \mathrm{c}$ had a polarization of $+0.044 \pm 0.008$ and a sample of $5 \times 10^{4}$ events with an average momentum of 304 . GeV/c had a polarization of $+0.036 \pm 0.015$. The polarization of $7.1 \times 10^{5} \Xi^{-}$s produced by a polarized neutral beam was $-0.118 \pm 0.004$ at an average momentum of $393 . \mathrm{GeV} / \mathrm{c} .1 .8 \times 10^{4} \Omega^{-} \mathrm{s}$ produced by the polarized neutral beam had a polarization of $-0.069 \pm 0.023$ at an average momentum of 394. GeV/c. The measurements for production from a polarized neutral beam are in agreement with a previous measurement.

\section{Acknowledgements}

This thesis could not have been completed without the assistance of many people. My fellow graduate students, Jerry Guglielmo and Noah Wallace, deserve a great deal of credit for the successful completion of this experiment. Jerry managed to keep the data acquisition system working and was always there to explain the intricacies of the polarization analysis software. Noah succeeded in keeping the wire chambers running, despite the fact that some of them were nearly twenty years old and were showing their age, and also sorted out the Monte Carlo software and kept it running.

My advisor, Ken Heller, deserves thanks for a number of things. He got the experiment approved and was a great source of knowledge about strange problems we encountered that had been solved by previous experiments. Ken Johns provided ideas, assistance, and encouragement throughout the whole experiment. In addition, the example he set through the dedication and energy he brought to the experiment were a continual source of inspiration. Gina Rameika deserves credit for getting the beamline running and helping us get support from the Fermilab staff. Pete Border was a constant source of wisdom about wire chambers and other types of experimental hardware. Dave Ciampa provided invaluable assistance with many parts of the experiment, especially the reconstruction software. Eileen Berman provided answers and assistance for wide variety of computing questions. Graham Allan and Ron Poling maintained and upgraded the computer system, without which this analysis would not have been possible. 
My fellow graduate students in the University of Minnesota High Energy Physics group deserve thanks for their patience when I was using most of the available CPU time on this computer system. The entire Fermilab staff deserves credit for their assistance in setting up and running the experiment.

In addition to the people who provided technical and scientific assistance to me during my time in graduate school, I benefitted greatly from personal relationships with many people. First and foremost are my family, who have supported and encouraged me throughout my life. They also provided the genes(see references 1 and 2), which seem to have helped. Thanks to my father and grandfather, who inspired me and showed me what fun physics could be from an early age. My sister Ann, who lived in the same city as me through much of my time in graduate school, deserves special credit for putting up with me for so long.

Thanks to Jerry and Noah for making work fun and not working even more fun. Eileen deserves thanks for being a most excellent source of suggestions of fun and interesting things to do. Ken Johns shared many late night shifts with me and made them fun and productive. Pete provided interesting music to listen to and fun toys to play with during shifts, and also told us when it was time to go to lunch. Dave Ciampa provided advice about how life and the world really work. Graham Allan was always ready to offer witty commentary on life in the physics department. Thanks also to Alex Ayala-Mercado, John Capriotti, Dave Fein, Milena Groblewski-Higgins, Eric James, Krista Mullman, and Tricia Tynan for their contributions. Thanks also to all the people I'm sure I've neglected to mention.

Thanks also to Bill Watterson and Universal Press Syndicate for granting me permission to reprint the Calvin and Hobbes cartoons in my thesis, and also for providing a constant source of amusement.
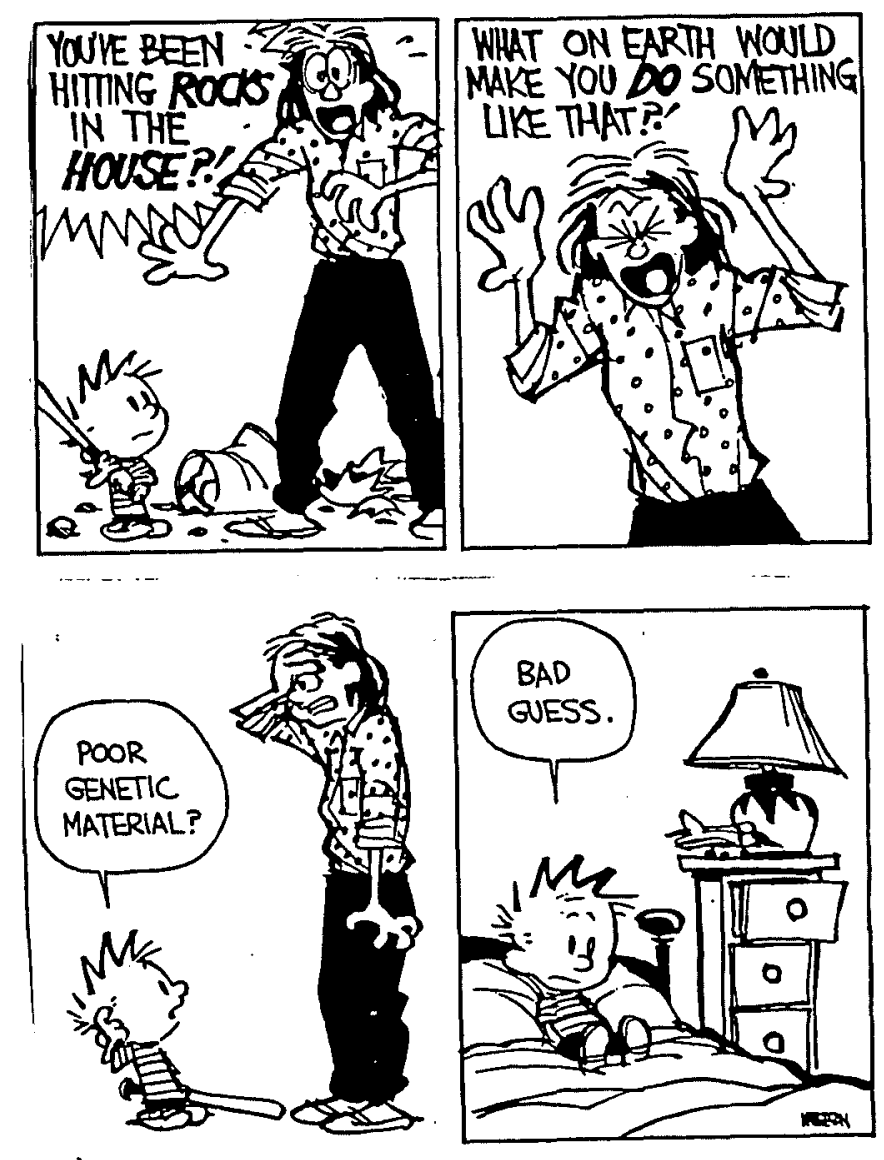

CALVIN AND HOBBES copyright Watterson. Dist. by UNIVERSAL PRESS SYNDICATE. Reprinted with permission. All rights reserved. 


\section{Dedication}

This dissertation is dedicated to my father and grandfather, who inspired me and showed me what fun science could be. 


\section{Table of Contents}

\begin{tabular}{|c|c|c|}
\hline \multicolumn{3}{|c|}{ Abstract } \\
\hline \multicolumn{3}{|c|}{ Acknowledgements } \\
\hline \multicolumn{3}{|c|}{ Dedication } \\
\hline \multicolumn{3}{|c|}{ List of Tables } \\
\hline \multicolumn{3}{|c|}{ List of Figures } \\
\hline \multicolumn{3}{|c|}{1 Introduction } \\
\hline & 1.1 Hyperon Polarization ..... . & $\ldots \ldots \ldots \ldots \ldots \ldots \ldots$ \\
\hline & 1.2 Previous Polarization Results & $\ldots \ldots \ldots \ldots \ldots \ldots$ \\
\hline & 1.3 Polarization Theory . . . . . & $\ldots \ldots$ \\
\hline \multicolumn{3}{|c|}{2 Apparatus } \\
\hline & 2.1 The Beamline $\ldots \ldots \ldots \ldots$ & $\ldots \ldots \ldots \ldots \ldots \ldots$ \\
\hline & 2.2 Targets and Sweeping Magnets & $\ldots \ldots \ldots \ldots \ldots \ldots$ \\
\hline & 2.3 Production Methods . & . \\
\hline & 2.4 Coordinate System . . . . . . & $\ldots \ldots \ldots \ldots \ldots \ldots$ \\
\hline & 2.5 The Spectrometer & \\
\hline
\end{tabular}

2.5.1 The Silicon Strip Detectors . . . . . . . . . 26

2.5.2 The Scintillation Counters . . . . . . . . . 27

2.5.3 The Multiwire Proportional Chambers . . . . . . . . 27

2.5.4 The Analyzing Magnets . . . . . . . . . . . 29

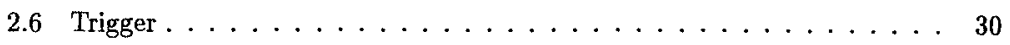

2.7 Data Acquisition System and Experimental Operation ........ 31

3 Event Reconstruction and Selection $\quad 36$

3.1 Introduction . . . . . . . . . . . . . . 36

3.2 The First Pass Analysis $\ldots \ldots \ldots \ldots \ldots \ldots \ldots$

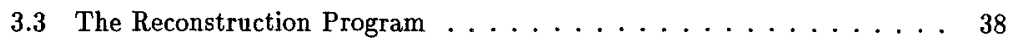

3.3.1 Initial Reconstruction Program $\ldots \ldots \ldots \ldots \ldots$

3.3.2 First Reconstruction Retry . . . . . . . . . . 41

3.3.3 Second Retry Method . . . . . . . . . . . . 42

3.4 The Monte Carlo Program . . . . . . . . . . . . . . . 43

3.5 Monte Carlo Program Performance . . . . . . . . . . . . 45

3.6 Performance of the Reconstruction Process $\ldots \ldots \ldots \ldots . \ldots 45$

4 Polarization Analysis $\quad 54$

$4.1 \Xi^{-}$Polarization . . . . . . . . . . . . . . . . . . 54

$4.2 \Omega^{-}$Polarization . . . . . . . . . . . . . . . . . 55

$4.3 \wedge$ Polarization Measurement . . . . . . . . . . . . . 56

4.4 The Bias Cancellation . . . . . . . . . . . . . . 59

4.5 Calculation of Target Polarization $\ldots \ldots \ldots \ldots \ldots$

5 Results and Systematic Uncertainty Studies $\quad 63$

5.1 Polarization Results ................ 63 
5.1.1 Unpolarized Neutral Beam Production Data. . . . . . . 63

5.1.2 Polarized Neutral Beam Production Data . . . . . . . . . 71

5.1 .3 Discussion of Biases $\ldots \ldots \ldots \ldots \ldots 71$

5.2 Systematic Studies . . . . . . . . . . . . . 81

5.2 .1 Single-track Triggers $\ldots \ldots \ldots \ldots \ldots \ldots$. . . . . 81

$5.2 .2 \quad$ Data at $0 \mathrm{mrad}$ production angle. . . . . . . 81

5.2 .3 Other Systematic Studies . . . . . . . . . . 85

5.3 Summary of Results $\ldots \ldots \ldots \ldots \ldots \ldots \ldots$

6 Conclusions $\quad 95$

6.1 Comparision With Previous Measurements .......... 95

6.2 Theoretical Predictions . . . . . . . . . . . . . . 95

6.2 .1 Other possible explanations . . . . . . . . . . . 101

6.3 Summary . . . . . . . . . . . . . . . . . . 102

Appendix A. Calculation of Polarization prediction for $\Xi^{0} \rightarrow \Xi^{-} \quad 105$

Appendix B. Hybrid Monte Carlo Topological Selection Criteria

109

References

111

\section{List of Tables}

1.1 The baryon SU(6) spin/flavor wave functions. . . . . . . . 10

1.2 Polarization predictions of the model by DeGrand and Miettinen. . . . 11

1.3 Polarization predictions of the model by DeGrand and Miettinen with

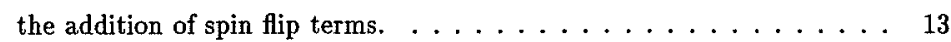

2.1 The size and $z$ positions of the spectrometer elements. . . . . . 25

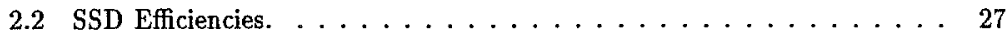

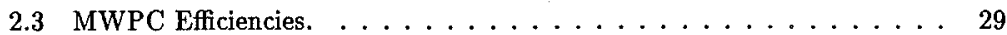

2.4 Summary of data collected. . . . . . . . . . . . 33

3.1 Selection criteria for the first pass reconstruction. . . . . . . . . 37

3.2 Summary of data remaining after first pass. . . . . . . . . 37

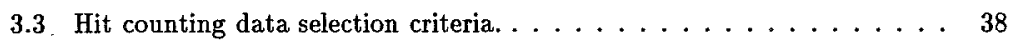

3.4 Reconstructed data selection criteria. . . . . . . . . . . . . . 41

3.5 Summary of data remaining after the reconstruction pass. . . . . . 43

5.1 Measured $\Xi^{-}$asymmetries and biases as a function of momentum for the unpolarized neutral beam production sample with the sweeper magnet set at -2900 amps. . . . . . . . . . . . . . . . . . . 64

5.2 Measured $\Omega^{-}$asymmetries and biases as a function of momentum for the unpolarized neutral beam production sample with the sweeper magnet set at -2900 amps. . . . . . . . . . . . . . . . 
5.3 Measured $\Xi^{-}$asymmetries and biases as a function of momentum for the unpolarized neutral beam production sample with the sweeper magnet set at -750 amps. . . . . . . . . . . . . . . 67

5.4 Measured $\Omega^{-}$asymmetries and biases as a function of momentum for the unpolarized neutral beam production sample with the sweeper magnet set at -750 amps. . . . . . . . . . . . . . . 68

5.5 Overall unpolarized neutral beam production $\Xi^{-}$sample asymmetries and biases for the two sweeper magnet settings. . . . . . . . 68

5.6 Overall unpolarized neutral beam production $\Omega^{-}$sample asymmetries and biases for the two sweeper magnet settings. . . . . . . . 68

5.7 Measured $\Xi^{-}$asymmetries and biases as a function of momentum for the polarized neutral beam production sample. . . . . . . . 73

5.8 Measured $\Omega^{-}$asymmetries and biases as a function of momentum for the polarized neutral beam production sample. . . . . . . . 73

5.9 Overall $\Xi^{-}$sample asymmetries and biases for the polarized neutral beam production sample. . . . . . . . . . . . 74

5.10 Overall $\Omega^{-}$sample asymmetries and biases for the polarized neutral beam production sample. . . . . . . . . . . . . . 74

5.11 Fitted target polarizations for all data samples. . . . . . . . . 74

5.12 Measured $\Xi^{-}$asymmetries and biases as a function of momentum for the single-track unpolarized neutral beam production sample with the sweeper magnet set at -2900 amps. . . . . . . . . . . . . 82

5.13 Measured $\Xi^{-}$asymmetries and biases as a function of momentum for data with no production angle. . . . . . . . . 85 5.14 Normal and "tighter" data selection criteria. . . . . . . . . . . 89

6.1 Predicted polarizations for production from an unpolarized neutral beam. 99
6.2 Predicted polarizations for production from a polarized neutral beam. . 100 B.1 Summary of hits required in the hybrid Monte Carlo polarization analysis.110 B.2 Summary of track separations required in the hybrid Monte Carlo polarization analysis. . . . . . . . . . . . . . 110 


\section{List of Figures}

1.1 Production of a polarized hyperon sample from an incident proton beam. 4

1.2 Polarization as a function of momentum at $400 \mathrm{GeV}$ incident beam energy. 5

1.3 Polarization as a function of $p_{T}$ at $400 \mathrm{GeV}$ incident beam energy. . . 6

1.4 Polarization as a function of $x_{F}$ at $400 \mathrm{GeV}$ incident beam energy. . . . 6

1.5 Polarization as a function of momentum at $800 \mathrm{GeV}$ incident beam energy. 7

1.6 Polarization results for the spin transfer method at $800 \mathrm{GeV}$. . . . 8

2.1 The downstream end of the beamline. . . . . . . . 17

2.2 The neutral collimator in PC3SW. . . . . . . . . . 18

2.3 The charged collimator in PC3ANA. . . . . . . . . 20

2.4 The proton production mode. . . . . . . . . . . . . 21

2.5 The unpolarized neutral beam production mode. . . . . . . . . 22

2.6 The polarized neutral beam production mode. . . . . . . . . 22

2.7 Plan view of the E800 spectrometer. . . . . . . . . . . 24

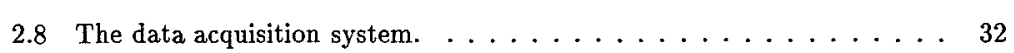

3.1 The reconstruction process. . . . . . . . . . . . . 39

3.2 Comparision of $\Xi^{-}$mass and momentum for Monte Carlo and real data. 46

3.3 Comparision of Monte Carlo and real data target distributions. . . . . 47
3.4 Comparision of $\Xi^{-}$and $\Lambda^{0}$ vertex distributions for Monte Carlo and real data. . . . . . . . . . . . . . . 48

3.5 Comparision of $\chi_{G}^{2} / D F$ and $\chi_{\Lambda}^{2}$ distributions for Monte Carlo and real data. . . . . . . . . . . . . . . . . 49

3.6 Difference between generated and reconstructed cosine values using only the first reconstruction process. . . . . . . . . . . . 51

3.7 Difference between generated and reconstructed cosine values using all parts of the reconstruction process. . . . . . . . . . 52

5.1 Asymmetries ( $\alpha_{\Lambda} \gamma_{\Xi} P_{\Xi}$ and $\alpha_{\Lambda} P_{\Omega}$ ) for the unpolarized neutral beam production sample with the sweeper magnet set at -2900 Amps. . . . . . 65

5.2 Biases for the unpolarized neutral beam production sample with the sweeper magnet set at -2900 Amps. . . . . . . . . . 66

5.3 Asymmetries $\left(\alpha_{\Lambda} \gamma_{\xi} P \Xi\right.$ and $\left.\alpha_{\Lambda} P_{\Omega}\right)$ for the unpolarized neutral beam production sample with the sweeper magnet set at -750 Amps. . . . . . 69

5.4 Biases for the unpolarized neutral beam production sample with the sweeper magnet set at -750 Amps. . . . . . . . . . . . 70

5.5 Measured precession angles for the unpolarized neutral beam production sample with the sweeper magnet set at -2900 Amps. . . . . . . . 72

5.6 Asymmetries $\left(\alpha_{\Lambda} \gamma \Xi P_{\Xi}\right.$ and $\left.\alpha_{\Lambda} P_{\Omega}\right)$ for the polarized neutral beam production sample. . . . . . . . . . . . . . 75

5.7 Biases for the polarized neutral beam production sample. . . . . . 76

5.8 Measured precession angles for the polarized neutral beam production sample. . . . . . . . . . . . . . 77

5.9 Fitted target polarizations for all samples. . . . . . . . . . 78

5.10 Comparision of biases for all the $\Xi^{-}$samples. . . . . . . . . 80 
5.11 Comparision of $\Xi^{-}$asymmetries $\left(\alpha_{\Lambda} \gamma \Xi P \Xi\right)$ for the normal and single-

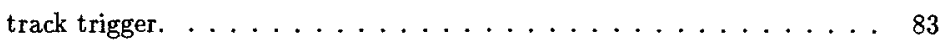

5.12 Comparision of $\Xi^{-}$biases for the normal and single-track trigger. . . . . 84

5.13 Comparision of $\Xi^{-}$asymmetries $\left(\alpha_{\Lambda} \gamma_{\Xi} P_{\Xi}\right)$ for the unpolarized neutral

beam production and $0 \mathrm{mrad}$ production. . . . . . . 86

5.14 Comparision of $\Xi^{-}$biases for unpolarized nentral beam production and

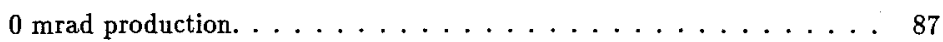

5.15 Comparision of $\Xi^{-}$asymmetries $\left(\alpha_{\Lambda} \gamma_{\Xi} P_{\Xi}\right)$ for unpolarized neutral beam

production and unpolarized Monte Carlo. . . . . . . . . . 88

$5.16 \Xi^{-}$asymmetries $\left(\alpha_{\Lambda} \gamma_{\Xi} P_{\Xi}\right)$ for seven unpolarized neutral beam produc-

tion data subsamples. . . . . . . . . . . . . . 90

$5.17 \Xi^{-}$biases for seven unpolarized neutral beam production data subsamples. 91

5.18 Measured $\Xi^{-}$magnetic moment for various "tighter" data selection criteria. 92

6.1 Comparision of $\Xi^{-}$polarization results. . . . . . . . . . 96

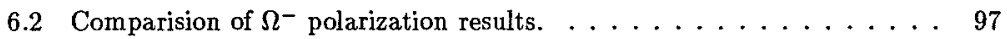



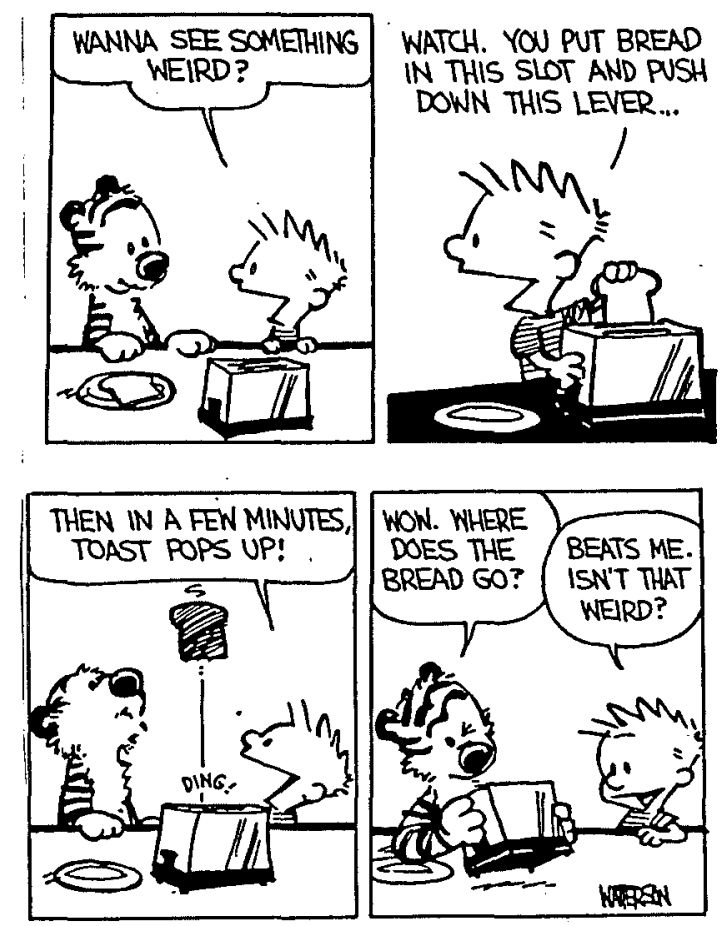

CALVIN AND HOBBES copyright Watterson. Dist. by UNIVERSAL PRESS SYNDICATE. Reprinted with permission. All rights reserved.

\section{Chapter 1}

\section{Introduction}

For many years, physicists have studied the interaction of particles to understand the basic forces of nature. These investigations have progressed to higher energies as the particles being studied have become smaller. In the early part of this century, atomic spectra were studied to understand the interaction between electrons and nuclei in atoms[1]. Later came the study of nuclear spectra to understand the structure of the nucleus[2]. Now, we study quarks and leptons to understand the fundamental forces of nature. The purpose of this experiment was to learn about quark interactions by studying the production of polarized hyperons.

\subsection{Hyperon Polarization}

Hyperon polarization in inclusive proton production reactions was first observed in a $\Lambda^{0}$ production experiment at Fermilab in 1976[3]. Since then, extensive experimental and theoretical efforts have been made in an attempt to explain this phenomena. Despite these efforts, a simple, concise explanation of the cause of polarization in hyperon 
production has not been found. In addition to the $\Lambda^{0}$, high energy polarization measurements have been made on many other baryons produced in proton interactions, including the proton, $\bar{\Lambda}, \Sigma^{+}, \Sigma^{0}, \Sigma^{-}, \bar{\Sigma}^{-}, \Xi^{-}, \Xi^{0}, \Xi^{+}$, and $\Omega^{-}[4,5,6,7,8,9,10,11,12,13]$. These polarization measurements are a sensitive way of studying quark interactions since they reflect the interference between the spin dependent and spin independent parts of the production process. There are many different ways of studying polarization phenomena, including production by polarized and unpolarized incident beams. The fact that the observed effects are large indicates that spin contributions are an important part of the strong interaction.

The technique used by most previous experiments to produce polarized samples is shown in Figure 1.1. In this method, a beam of unpolarized particles strikes a target from a known direction. In Figure $1.1, \hat{i}$ is the direction of the incident beam, $\hat{j}$ is the direction of the produced particle, and $\theta$ is the production angle. The only parity conserving direction in this interaction is perpendicular to the production plane defined by $\hat{i} \times \hat{j}$. Since spin is invariant under a parity transformation, and the strong force that governs this interaction conserves parity, the spin of the produced particles must be perpendicular to the production plane.

Experiments have also been conducted using incident $\pi, \Lambda, \mathrm{K}$, and $\nu$, and $\gamma$ beams[14, $15,16,17,18]$. There have been other experiments that have used polarized proton beams to study spin transfer process[19,20,21]. In addition, $\Lambda$ polarization has been observed in a pp collider experiment[22]

One experiment has used the proton production technique to produce a secondary polarized neutral beam which was then used to produce a tertiary negatively charged beam. This experiment studied how the polarization of the secondary beam was transferred to the tertiary beam. This technique was used to produce polarized samples of $\Omega^{-}$hyperons that were not polarized when produced directly from protons[13].

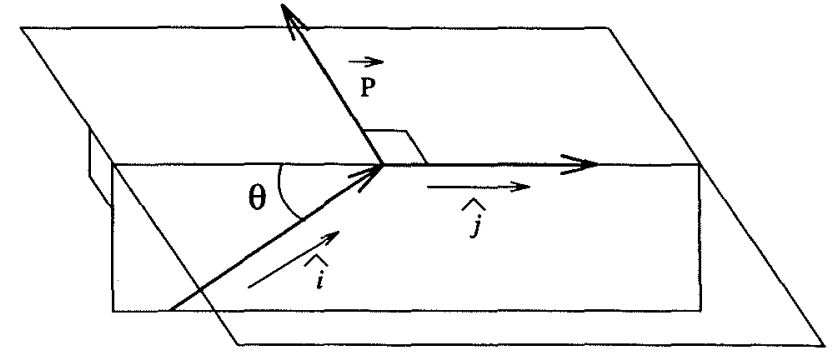

Figure 1.1: Production of a polarized hyperon sample from an incident proton beam.

\subsection{Previous Polarization Results}

Any inclusive reaction can be characterized by three kinematic variables: beam energy, transverse momentum $\left(p_{T}\right)$, and longitudinal momentum or Feynman $x\left(x_{F}\right)$. These quantities are given by:

$$
\begin{gathered}
p_{T}=p \sin \theta \\
x_{F}=\frac{p l^{*}}{p !_{\max }{ }^{*}}
\end{gathered}
$$

where $\theta$ is the production angle, $p$ is the momentum of the particle produced in the interaction, $p^{*}$ is the longitudinal momentum of the produced particle in the center of mass frame, and $p_{l \text { max }}{ }^{*}$ is the maximum longitudinal momentum. For the results presented here, where the produced particle is in the forward direction, and the energy is much greater than the mass, $x_{F}$ can be approximated by

$$
x_{F}=\frac{P_{\text {produced }}}{P_{\text {beam }}}
$$

where $P_{\text {produced }}$ and $P_{\text {beam }}$ are the laboratory frame momenta of the produced hyperon and the incident beam respectively.

Figure 1.2 shows previous polarization results as a function of momentum for 400 $\mathrm{GeV}$ protons producing $\Lambda \mathrm{s}, \bar{\Lambda} \mathrm{s}, \Sigma^{-} \mathrm{s}, \Xi^{0} \mathrm{~s}$, and $\Xi^{-} \mathrm{s}$ at a production angle of $7.5 \mathrm{mrad}[5$, $7,10,11,23]$. Note that the sign of the polarization of the $\Lambda \mathrm{s}, \Xi^{0} \mathrm{~s}$, and $\Xi^{-} \mathrm{s}$ is opposite 


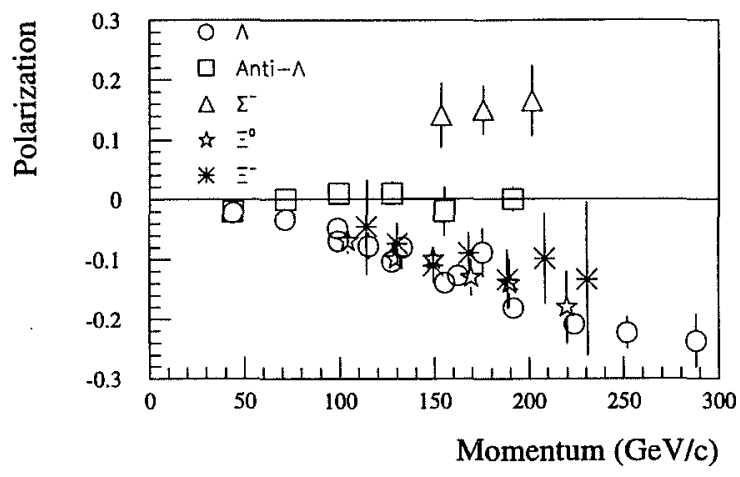

Figure 1.2: Polarization as a function of momentum at $400 \mathrm{GeV}$ incident beam energy.

the sign of the polarization of the $\Sigma^{-} s$, and that the $\bar{\Lambda} s$ are unpolarized. Data at other production angles show that $\Sigma^{+} s$ and $\Sigma^{0} s$ are polarized with the same sign as the $\Sigma^{-} s[6,8]$.

Figures 1.3 and 1.4 show these same polarization results as a function of $p_{T}$ and as a function of $x_{F}[5,7,10,23] . \Lambda^{0}$ polarization has been studied at a variety of energies and no significant energy dependence has been found. In addition, studies of $\Lambda^{0}$ production from a variety of different nuclear targets show no variation [23]. Similar studies for the polarization of proton produced $\Xi^{0} \mathrm{~s}$ show no target dependence [11].

A more recent experiment made a detailed study of polarization for $\Xi^{-} \mathrm{s}$ [24]. These results show that $P_{\Xi-}$ and $P_{\Lambda}$ have similar behavior as a function of $p_{T}$, but their $x_{F}$ behavior is different. In addition, comparison of these results, which were obtained with $800 \mathrm{GeV}$ incident protons, to $400 \mathrm{GeV}$ data with similar $p_{T}$ and $x_{F}$, showed that unlike $P_{\Lambda}, P_{\Xi-}$ does depend on the incident beam energy.

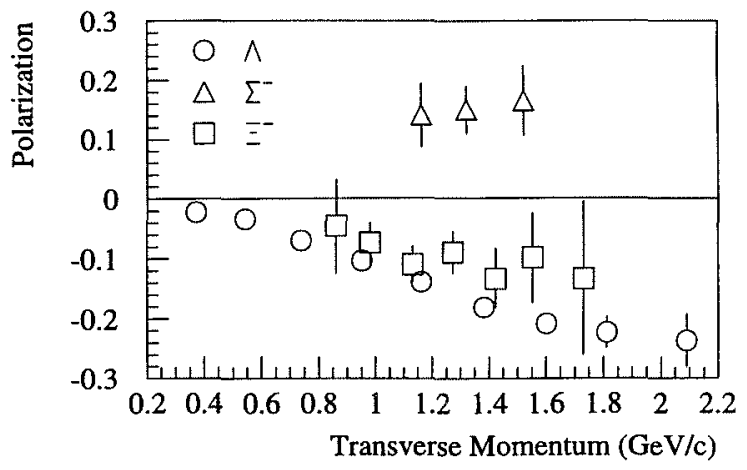

Figure 1.3: Polarization as a function of $p_{T}$ at $400 \mathrm{GeV}$ incident beam energy.

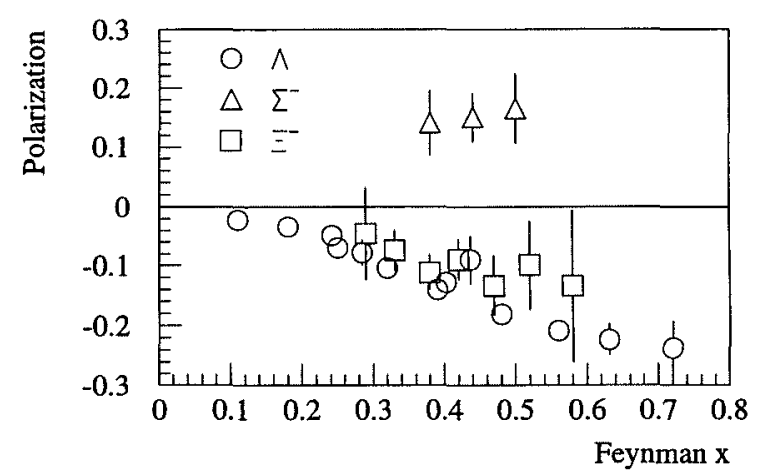

Figure 1.4: Polarization as a function of $x_{F}$ at $400 \mathrm{GeV}$ incident beam energy. 


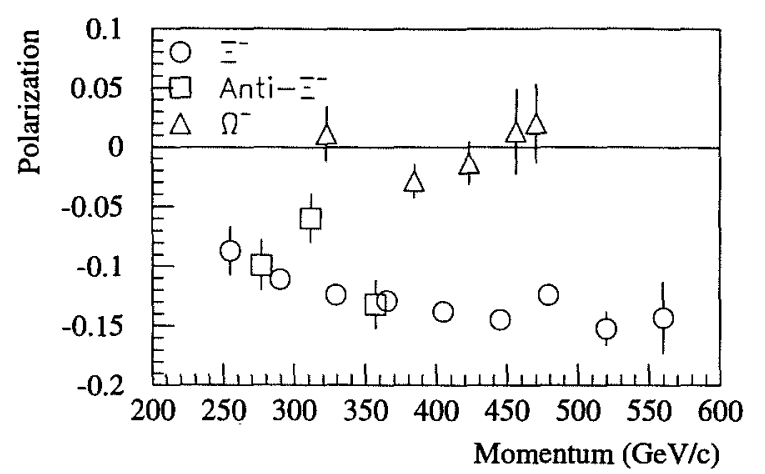

Figure 1.5: Polarization as a function of momentum at $800 \mathrm{GeV}$ incident beam energy.

This same experiment also studied the polarization of $\Omega^{-} \mathrm{s}$, and $\bar{\Xi}^{+} \mathrm{s}$ produced using an $800 \mathrm{GeV}$ incident proton beam[12,13,24]. A comparison of $\Xi^{-}, \Omega^{-}$, and $\bar{\Xi}^{+}$ polarization at a production angle of $2.5 \mathrm{mrad}$ are shown in Figure 1.5. This was the first experiment to observe anti-hyperon polarization. In addition, it can be seen that $\Omega^{-}$s produced from protons are not polarized. This is the first hyperon where polarization has not been observed in proton production. Another recent result has shown that $\bar{\Sigma}^{-}$s produced from protons are also polarized[9].

Since $\Omega^{-}$s produced by a proton beam were observed to be unpolarized, another method was used to make polarized $\Omega^{-}$. In this method, referred to as spin transfer production, the proton beam was used to produce a neutral beam containing neutrons, $\gamma \mathrm{s}, K^{-0} \mathrm{~s}, \Lambda \mathrm{s}$ and $\Xi^{0} \mathrm{~s}$. The proton beam struck a target at a non-zero production angle, producing polarized $\Lambda \mathrm{s}$ and $\Xi^{0} \mathrm{~s}$ in the neutral beam. This neutral beam, which was basically a beam of polarized strange quarks, was then used to produce a sample of $\Xi^{-} \mathrm{s}$

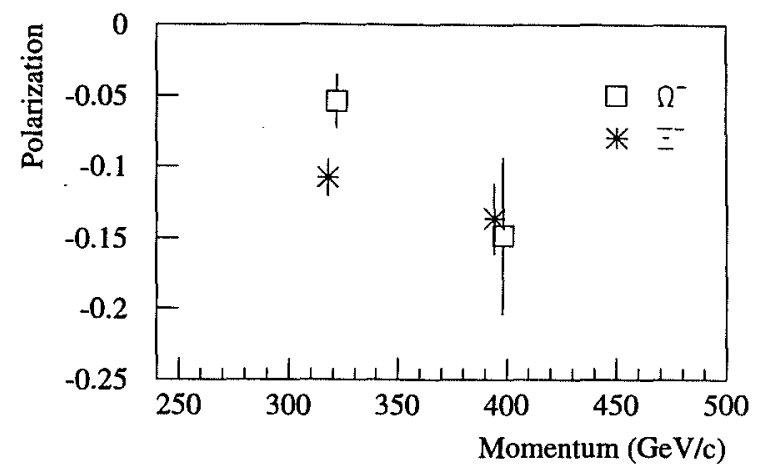

Figure 1.6: Polarization results for the spin transfer method at $800 \mathrm{GeV}$.

and $\Omega^{-}$s, with the expectation that the polarized strange quarks would be transferred to the $\Xi^{-} s$ and $\Omega^{-}$s. The results for this production method are shown in Figure 1.6. These results show that both the $\Xi^{-} \mathrm{s}$ and $\Omega^{-} \mathrm{s}$ produced by this method are polarized $[25,26]$. This spin transfer method was also studied by an earlier experiment that observed the transfer of polarization from a polarized proton beam to $\Lambda s$ and $\Sigma^{0} s[21]$.

\subsection{Polarization Theory}

There are several models that attempt to explain the observed polarization phenomena. Most of these models use quark ideas and assume that the hyperon is formed from a fragment of the incident particle and one or more quarks from the quark sea. For instance, $\Xi^{-}(d s s)$ production from protons can be seen as the $d$ quark from the incident proton combining with two $s$ quarks from the sea. 
Perturbative quantum chromodynamics ( $\mathrm{PQCD}$ ) has been used to calculate the transverse polarization of produced quarks with the result that

$$
P \propto \frac{m}{p_{T}^{2}}
$$

where $m$ is the quark mass, and $p_{T}$ is the transverse momentum[27]. However, this is a perturbative calculation that can only be applied to problems where the energy scale of the problem is much larger than $\Lambda_{Q C D}$, which has a value in the range 0.1 to 0.5 $\mathrm{GeV}$ [28]. Equation 1.4 predicts that the polarization should be zero at high transverse momentum. The highest $p_{T}$ value reached in polarization measurements is a study of $\Lambda^{0}$ polarization in a region up to $3.8 \mathrm{GeV} / \mathrm{c}$ [23], a region where the PQCD prediction is only marginally applicable. However, in the experimental measurements, there is no indication that the polarization is tending towards zero.

At the opposite end of the energy spectrum from the $\mathrm{pQCD}$ calculation is a naive quark model polarization theory, constructed using only the simple spin/flavor SU(6) wave functions for the baryons given in Table 1.1. Construction of this model was encouraged by the reasonable agreement between hyperon magnetic moments calculated using the simple quark model and experimental observations. The wave functions provide a simple explanation for the most striking property of hyperon polarization, namely the different signs of the measured polarizations. If it is assumed that the strong production mechanism always polarizes the $s$ quark in the same direction, the wave functions show that $\Sigma^{+} \mathrm{s}, \Sigma^{0} \mathrm{~s}$, and $\Sigma^{-} \mathrm{s}$ should have polarizations opposite to those of $\Lambda \mathrm{s}, \Xi^{-} \mathrm{s}, \Xi^{0} \mathrm{~s}$, and $\Omega^{-} \mathrm{s}$. If the proton beam is unpolarized, then the $u$ and $d$ quarks from the incident proton that are retained in the final state should have no preferred spin direction. From Table 1.1, it can be seen that for the spin $\frac{1}{2}$ strange hyperons, the wave functions can be seen as either a combination of a spin $1 u d$ diquark and a single $s$ quark or a combination of a spin $1 s s$ diquark with a single $u$ or $d$ quark. In either case, if only one direction is allowed for the $s$ quark spin, a direct correlation between

\begin{tabular}{c|c} 
Baryon & Quark wave function \\
\hline$p \uparrow$ & $\sqrt{2 / 3} u \uparrow u \uparrow d \downarrow-\sqrt{1 / 6}(u \uparrow u \downarrow+u \downarrow u \uparrow) d \uparrow$ \\
$n \uparrow$ & $\sqrt{2 / 3} d \uparrow d \uparrow u \downarrow-\sqrt{1 / 6}(d \uparrow d \downarrow+d \downarrow d \uparrow) u \uparrow$ \\
$\Lambda \uparrow$ & $\sqrt{1 / 2}(u \uparrow d \downarrow-u \downarrow d \uparrow) s \uparrow$ \\
$\Sigma^{+} \uparrow$ & $\sqrt{2 / 3} u \uparrow u \uparrow s \downarrow-\sqrt{1 / 6}(u \uparrow u \downarrow+u \downarrow u \uparrow) s \uparrow$ \\
$\Sigma^{0} \uparrow$ & $\sqrt{2 / 3} u \uparrow d \uparrow s \downarrow-\sqrt{1 / 6}(u \uparrow d \downarrow+u \downarrow d \uparrow) s \uparrow$ \\
$\Sigma^{-} \uparrow$ & $\sqrt{2 / 3} d \uparrow d \uparrow s \downarrow-\sqrt{1 / 6}(d \uparrow d \downarrow+d \downarrow d \uparrow) s \uparrow$ \\
$\Xi^{0} \uparrow$ & $\sqrt{2 / 3} s \uparrow s \uparrow u \downarrow-\sqrt{1 / 6}(s \uparrow s \downarrow+s \downarrow s \uparrow) u \uparrow$ \\
$\Xi^{-} \uparrow$ & $\sqrt{2 / 3} s \uparrow s \uparrow d \downarrow-\sqrt{1 / 6}(s \uparrow s \downarrow+s \downarrow s \uparrow) d \uparrow$ \\
$\Omega^{-} \uparrow$ & $s \uparrow s \uparrow s \uparrow$
\end{tabular}

Table 1.1: The baryon SU(6) spin/flavor wave functions.

the $s$ quark spin and the overall hyperon spin can be seen. In the $\Sigma$ wave functions, there are both spin up and spin down $s$ quark terms, however, the $s \downarrow$ term is larger. In the other hyperon wave functions, the $s \uparrow$ terms dominate.

One model hypothesizes that the final state polarization comes about during the recombination of the incident fragment and sea quarks to produce the final state. Other models account for the polarization by proposing methods by which the quarks produced from the sea have a preferred spin direction.

The most extensive model to date is that of DeGrand and Miettinen[29], who propose that the polarization is the result of a Thomas precession of the quark spins in the color field. The precession affects both the sea quarks and the quarks from the incident particle. This model also attempts to explain the transverse momentum $\left(p_{T}\right)$ and Feynman $\mathrm{x}\left(x_{F}\right)$ dependence observed in studies of $\Lambda$ polarization.

The latest version of this model also makes predictions for hyperons produced from polarized incident beams and for production from particles other than protons[30]. Table 1.2 lists these predictions as a function of $d, \epsilon, \epsilon^{\prime}, \delta$ and $\delta^{\prime}$. Here $d$ is the incident beam polarization, $\epsilon$ and $\delta$ are the polarization contributions of the single quark and diquark respectively for processes where the diquark comes from the incident particle, and $\epsilon^{\prime}$ and $\delta^{\prime}$ are the single quark and diquark contributions for processes where the 


\begin{tabular}{c|c} 
Interaction & Polarization \\
\hline$p \leftrightarrow \Lambda^{0}, n \leftrightarrow \Lambda^{0}$ & $-\epsilon$ \\
$n \leftrightarrow \Sigma^{+}, n \leftrightarrow \Sigma^{-}, p \leftrightarrow \Sigma^{0}, \Xi^{0} \leftrightarrow \Xi^{-}$ & $\frac{\epsilon+2 \delta+2 d}{3+(2 \epsilon+2 \delta) d}$ \\
\hline$p \leftrightarrow n, \Sigma^{+} \leftrightarrow \Xi^{0}, \Sigma^{-} \leftrightarrow \Xi^{-}$ & $\frac{-40 \epsilon+\delta+d}{42+(\epsilon+\delta) d}$ \\
$\Sigma^{ \pm} \leftrightarrow \Sigma^{0}, \Xi^{0} \leftrightarrow \Sigma^{0}$ & \\
\hline$\Sigma^{ \pm} \leftrightarrow \Lambda^{0}, \Xi^{0} \leftrightarrow \Lambda^{0}$ & $\frac{-4 \epsilon+\delta+d}{6+(\epsilon+\delta) d}$ \\
$\Xi^{-} \leftrightarrow \Lambda^{0}$ & \\
\hline$p \rightarrow \Sigma^{-}, n \rightarrow \Sigma^{+}, \Sigma^{+} \rightarrow \Xi^{-}$ & $\frac{12 \epsilon^{\prime}-3 \delta^{\prime}-4 d}{18+\left(-6 \epsilon^{\prime}-\delta^{\prime}\right) d}$ \\
$\Sigma^{-} \rightarrow \Xi^{0}, \Xi^{0} \rightarrow p, \Xi^{-} \rightarrow n$ & \\
\hline$p \leftrightarrow \Xi^{-}, n \leftrightarrow \Xi^{0}, \Sigma^{+} \leftrightarrow \Sigma^{-}$ & $\frac{-3 \epsilon^{\prime}-6 \delta^{\prime}+d}{9+\left(-3 \epsilon^{\prime}-2 \delta^{\prime}\right) d}$ \\
\hline$p \rightarrow \Xi^{0}, \Xi^{0} \rightarrow \Sigma^{-}, \Sigma^{-} \rightarrow p$ & $\frac{-3 \epsilon^{\prime}-6 \delta^{\prime}-2 d}{9+\left(6 \epsilon^{\prime}+4 \delta^{\prime}\right) d}$ \\
$n \rightarrow \Xi^{-}, \Xi^{-} \rightarrow \Sigma^{+}, \Sigma^{+} \rightarrow n$ & \\
\hline$\Sigma^{-} \rightarrow \Omega^{-}$ & $-\frac{5}{18} d+\frac{5}{6}(\epsilon-\delta)$ \\
\hline$\Xi^{0}, \Xi^{-} \rightarrow \Omega^{-}$ & $\frac{5}{6}(\delta-\epsilon+d)$ \\
\hline$\Lambda^{0} \rightarrow \Omega^{-}$ & $\frac{5}{6}(\epsilon-\delta+d)$ \\
\hline
\end{tabular}

Table 1.2: Polarization predictions of the model by DeGrand and Miettinen. $\epsilon, \epsilon^{\prime}, \delta$, and $\delta^{\prime}$ represent the polarization contribution of the single quark and diquarks and $d$ is the incident polarization.

diquark originates in the sea. Initially, DeGrand and Miettinen did not differentiate between $\epsilon$ and $\epsilon^{\prime}$ and $\delta$ and $\delta^{\prime}$, but there is evidence that suggests that they are not equal[20,31]. Experimental measurements require $\delta$ and $\epsilon$ to equal at the $20 \%$ level, with a value in the range $0.1-0.2$.

Bonner et al.[21] have modified the DeGrand and Miettinen model to allow for spin flips in the fragment from the incident particle. It was observed that the measured $\Lambda^{0}$ to $\Sigma^{0}$ production ratio, which is spin dependent, was noticeably different from those predicted by the DeGrand and Miettinen model [32]. The introduction of the spin flip terms was used to make predictions that were in closer agreement with the experimental observations. In this model there is some probability, $\mathcal{P}$, that the spin of a single quark will flip. This adds two parameters $\tau_{1}$ and $\tau_{2}$ to the model.

$$
\begin{gathered}
\tau_{1}=\frac{\mathcal{P}}{1-\mathcal{P}} \\
\tau_{2}=\frac{2 \mathcal{P}(1-\mathcal{P})}{1-2 \mathcal{P}(1-\mathcal{P})}
\end{gathered}
$$

$\tau_{1}$ is the parameter for interactions where only one quark from the incident particle is present in the final state, and $\tau_{2}$ is the parameter for cases when a diquark from the initial particle is present in the final state. Bonner et al. report a value of $\tau_{2}=$ $0.38 \pm 0.04$ is needed to fit the observed ratio of the cross-sections for production of $\Sigma^{0} \mathrm{~s}$ and $\Lambda^{0}$ s from protons. This results in a spin flip probability, $\mathcal{P} \approx 0.16$, and a value of $\tau_{1} \approx 0.19$. Table 1.3 gives predictions for hyperon polarization with the spin flip terms included for the interactions of interest to this experiment. A detailed example of these calculations is given in Appendix A. It should be possible to predict the expected final state polarization using the predictions given in Table 1.3. This will be discussed further in Chapter 6.

A model proposed by Andersson, Gustafson, and Ingelman[33], known as the Lund model, uses the production of $s \bar{s}$ pairs from the sea through a tunneling process to explain $\Lambda$ polarization. This is a soft process where PQCD is not applicable. The tunneling process gives rise to an enhancement in the number of hyperons produced with their $p_{T}$ in the same direction as the $p_{T}$ of the $s$ quark. In this process, the $p_{T}$ of the $s$ quark is related to its spin through the conservation of angular momentum. It is possible to extend the idea of this model to the production of anti-hyperons. If the idea of the production of a quark - anti-quark pair is extended to the production of a hyperon - anti-hyperon pair, it would predict that the sign of the polarization of 


\begin{tabular}{c|c} 
Interaction & Polarization \\
\hline$n \rightarrow \Xi^{-}$ & $\frac{2 d\left(\tau_{1}-1\right)-\left(3 \epsilon^{\prime}+6 \delta^{\prime}\right)\left(1+\tau_{1}\right)}{d\left(1-\tau_{1}\right)\left(6 \epsilon^{\prime}+4 \delta^{\prime}\right)+9\left(1+\tau_{1}\right)}$ \\
$\Lambda \rightarrow \Xi^{-}$ & $\frac{d-4 \epsilon+\delta\left(1+\tau_{2}\right)}{d(\epsilon+\delta)+6+2 \tau_{2}}$ \\
$\Xi^{0} \rightarrow \Xi^{-}$ & $\frac{2 d+\epsilon+2 \delta\left(1+\tau_{2}\right)}{2 d(\delta+\epsilon)+3+4 \tau_{2}}$ \\
$n \rightarrow \Omega^{-}$ & 0 \\
$\Lambda \rightarrow \Omega^{-}$ & $\frac{5}{6} \frac{d+\epsilon^{\prime}-\delta^{\prime}+\tau_{1}\left(\epsilon^{\prime}-\delta^{\prime}-d\right)}{1+\tau_{1}}$ \\
$\Xi^{0} \rightarrow \Omega^{-}$ & $\frac{10(d-\epsilon+\delta)+2 \tau_{2}(5 \delta-6 \epsilon)}{10 d \delta-8 d \epsilon+12+14 \tau_{2}}$
\end{tabular}

Table 1.3: Polarization predictions of the model by DeGrand and Miettinen with the addition of spin flip terms.

anti-hyperons produced in this manner should be opposite that of the hyperon. This does not match the experimental observations.

Szwed [34] has proposed a model for $\Lambda$ polarization where the $s$ quark polarization is due to multiple scattering of the $s$ quark from the sea in the color field. This model also offers an explanation of the $p_{T}$ dependence of the $\Lambda$ and predicts no strong $x_{F}$ dependence in this process.

A model proposed by Dharamaratna and Goldstein [35] explores the idea that the strange quark from the sea is produced by the fusion of two gluons. They conclude that $s$ quarks produced by this process could be polarized, leading to the polarization of the produced $\Lambda$. They have calculated the dependence of $\Lambda$ polarization on $p_{T}$ and $x_{F}$, and these predictions agree with the experimental data. These calculations have not been extended to any other hyperons.

Barni et al. propose a model in which the final state $\Lambda^{0}$ can be produced by several processes including direct production, production from $\Sigma^{0}$ electromagnetic decays, and production from the decay of intermediate $\Sigma^{+}$and $\Sigma^{*}$ baryons [36]. In this model, the final state polarization is due to interference between these various production channels. This model should also be applicable to $\Sigma^{+}$and $\Sigma^{-}$production, however it is not clear that it can be extended to $\Xi^{0}$ and $\Xi^{-}$production.

A model proposed by Soffer [37] uses a pion exchange mechanism to explain $\Lambda$ polarization. Once again, the final state $\Lambda^{0}$ can be produced from several intermediate states, and interference between these different production channels is used to explain the observed final state polarization. It successfully reproduces the observed $p_{T}$ and $x_{F}$ dependence of the $\Lambda$ polarization. However, as with most of the other models, it has not been extended to any other processes.

While several models have been proposed to explain the observed hyperon polarization data, none of the models offers an acceptable explanation of all of the experimental data. The motivation of this experiment was to study hyperon polarization in a way that might offer further insight into the origin of this phenomena. To do this, $\Xi^{-}$and $\Omega^{-}$hyperons were made using three different techniques. In addition to proton production, $\Xi^{-} s$ and $\Omega^{-} s$ were made from both a polarized and an unpolarized neutral beam. The unpolarized neutral beam production was of special interest since the neutral beam would contain $\Lambda^{0} s$ and $\Xi^{0} s$, and therefore, some of the strange quarks needed to produce the final state $\Xi^{-} s$ and $\Omega^{-} s$ would already be present. 

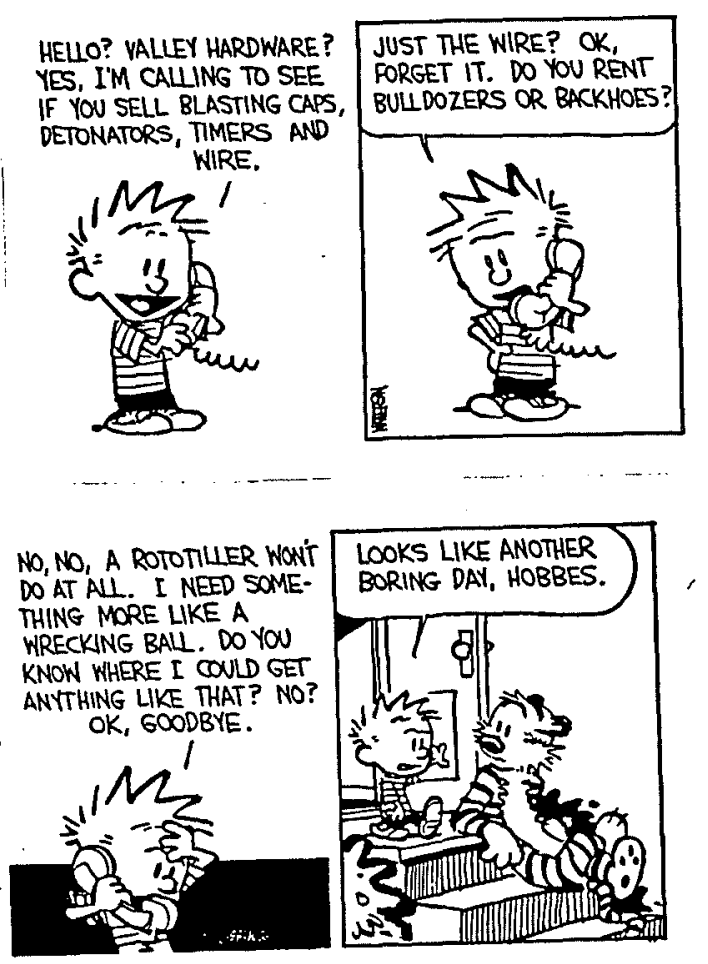

CALVIN AND HOBBES copyright Watterson. Dist. by UNIVERSAL PRESS

SYNDICATE. Reprinted with permission. All rights reserved.

\section{Chapter 2}

\section{Apparatus}

The data used in this experiment were collected as part of Experiment 800 (E800) at Fermi National Accelerator Laboratory (Fermilab) located in Batavia, Illinois, a suburb of Chicago. E800 took data during a fixed target run that began in June 1991 and ended in January 1992. The beamline used to transport the incident protons to the target and make the hyperons that were to be studied, the spectrometer that detected the hyperons and their decay products, and the data acquisition system used to record the data were the most important parts of the experimental apparatus and are described here in detail.

\subsection{The Beamline}

The accelerator, called the TeVatron, produced a beam of approximately $10^{13} 800 \mathrm{GeV}$ protons per spill. Each spill lasted 20 seconds with one spill per minute. The beamline used in this experiment was designed to transport a $800 \mathrm{GeV}$ proton beam with an intensity of up to $2.5 \times 10^{12}$ protons per spill.

The beamline contained dipole magnets used for horizontal and vertical beam positioning. It also had horizontal and vertical focusing quadrupole magnets. These 


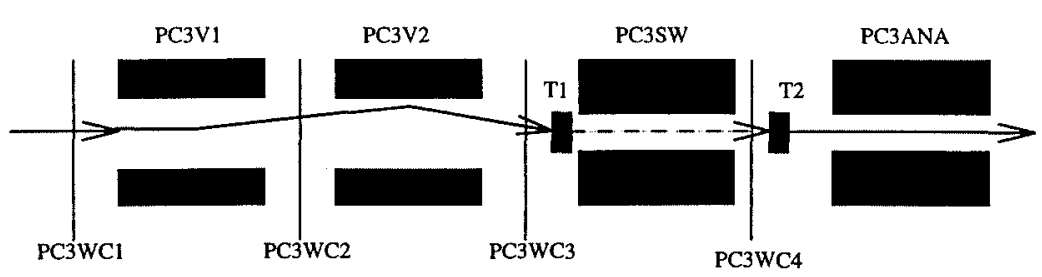

Figure 2.1: The downstream end of the beamline. This shows the beamline as it was configured for polarized neutral beam production.

magnets focused the beam so that it had a diameter of less than $2 \mathrm{~mm}$ when it struck the target.

\subsection{Targets and Sweeping Magnets}

The different production modes used in this experiment required a high degree of flexibility in the downstream end of the beamline where the target stations were located. The downstream end of the beamline contained the magnets used to determine the angle at which the proton beam struck the upstream target. The production angles used were $0 \mathrm{mrad}$ and $\pm 1.8 \mathrm{mrad}$. The size of the production angle was mainly limited by the magnets available to bend the protons away from the centerline and then bring the beam back onto the target. The production angle was chosen to maximize the polarization and production rate, since as the production angle increased, the production rate decreased. Figure 2.1 is a schematic drawing of the downstream end of the beamline. The configuration of the beamline for the various production mode will be discussed in section 2.3. Dipole magnets PC3V1 and PC3V2 were used to control the production angle. Magnet PC3V1 was used to bend the protons up or down and magnet PC3V2 was used to bring the beam back onto the target.

PC3SW was a standard main ring B2 dipole magnet that was used to select a neutral beam. It had a field strength of 1.8 Tesla in the horizontal direction and was $6 \mathrm{~m}$ long.
Plan View

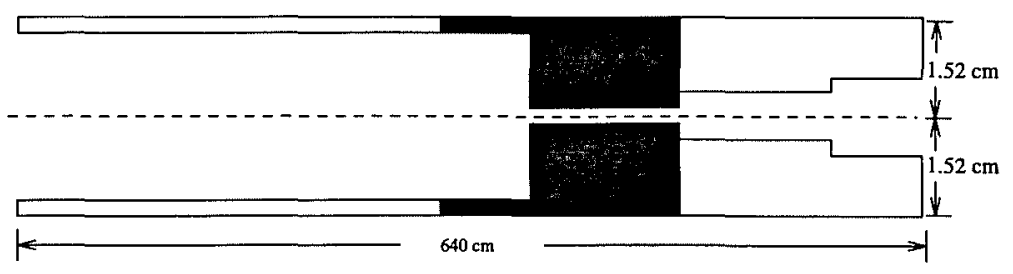

Elevation View

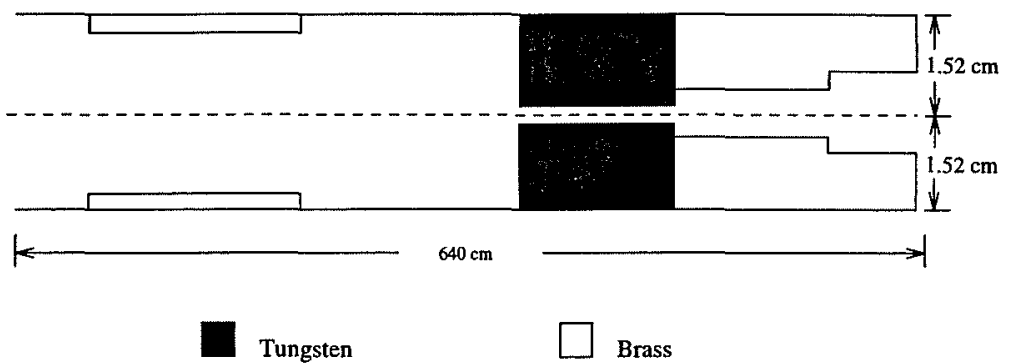

Figure 2.2: The neutral collimator in PC3SW.

The field direction was chosen to be in the horizontal direction to ensure that it would not precess the spin of the polarized particles that were transported through this magnet in one production mode. It was mounted on remotely controlled motorized jacks that allowed both ends of the magnet to be raised and lowered when the production angle was changed. Magnet $\mathrm{PC} 3 \mathrm{SW}$ was used as both a proton beam dump and a neutral beam selector. Figure 2.2 shows the collimator inside magnet PC3SW. This collimator was made of brass and tungsten, with the tungsten segments placed where the proton beam was expected to dump when the incident angle was between 0.0 and $2.0 \mathrm{mrad}$. The defining aperture of the collimator was $0.25 \mathrm{~cm}$ by $0.25 \mathrm{~cm}$.

Magnet PC3ANA, also referred to as the Hyperon magnet, was used to select a 
negatively charged beam. Figure 2.3 shows the charged collimator in the Hyperon magnet. This collimator was designed with a central orbit momentum of $400 \mathrm{GeV}$ at a magnetic field of 3.35 Tesla. Since this collimator was only designed to transport a tertiary beam of charged particles, it was constructed entirely of brass. The defining aperture of the collimator was $0.51 \mathrm{~cm}$ by $0.51 \mathrm{~cm}$. This magnet also served to precess the spin of the particles passing through it, which was necessary for the magnetic moment measurements that were also made in this experiment.

The two targets used were made of beryllium to maximize the fraction of high momentum baryons produced. Both were approximately 0.37 interaction lengths long. The upstream target, PC3TGT1, was a cylinder $150 \mathrm{~mm}$ long and $6.6 \mathrm{~mm}$ in diameter. It was mounted on the upstream end of PC3SW so that it moved in the vertical direction along with the magnet. The second target was $150 \mathrm{~mm}$ long and rectangular in shape, with a width of $5.2 \mathrm{~mm}$ and a height of $5.3 \mathrm{~mm}$. It was mounted on the upstream end of magnet PC3ANA. Both targets were mounted on motorized carriers with position sensors that allowed both targets to be moved horizontally. This allowed either target to be removed from the beam during the performance of background studies.

Segmented Wire Ion Chambers (SWICs) were used to monitor the beam position and thus determine the production angle. Three SWICs, PC3WC2, PC3WC3, and PC3WC4, each with $0.5 \mathrm{~mm}$ wire spacing, were used to monitor the position of the beam. The locations of these SWICs are shown in Figure 2.1. PC3WC2 was located between magnets PC3V1 and PC3V2 and was used to measure the production angle. PC3WC3 was located in front of the upstream target and was mounted on magnet PC3SW so that it moved with the magnet as it was raised or lowered. PC3WC4 was mounted in front of the second target.

A secondary emission monitor (SEM) was used to monitor the proton beam. The SEM provided a measure of the intensity of the proton beam in the Proton Center beamline. The SEM reading for each spill was recorded on our data tapes. Together,

Plan View

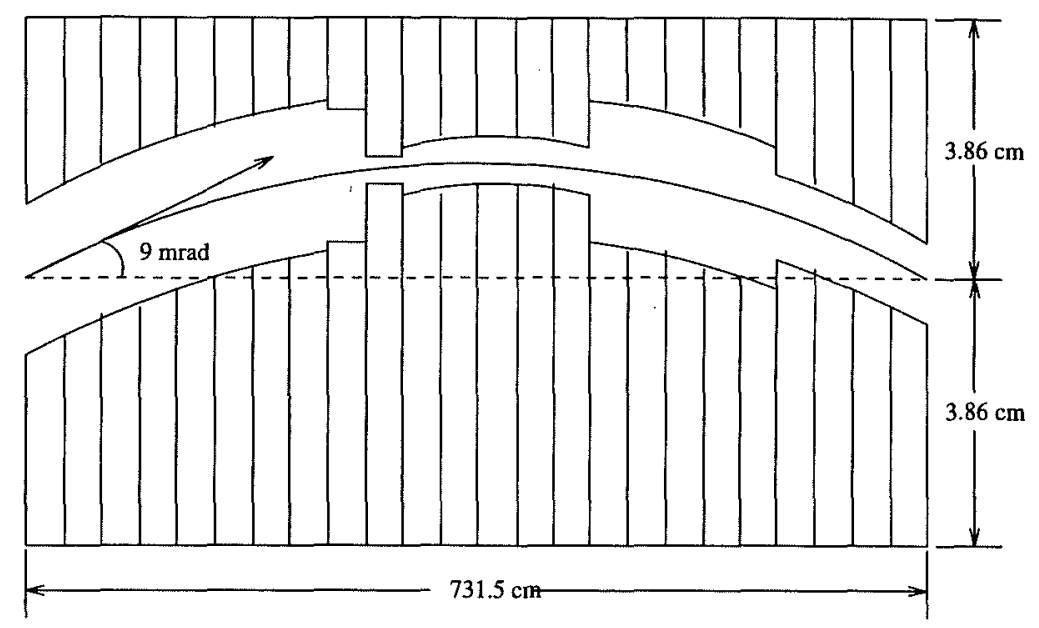

Elevation View

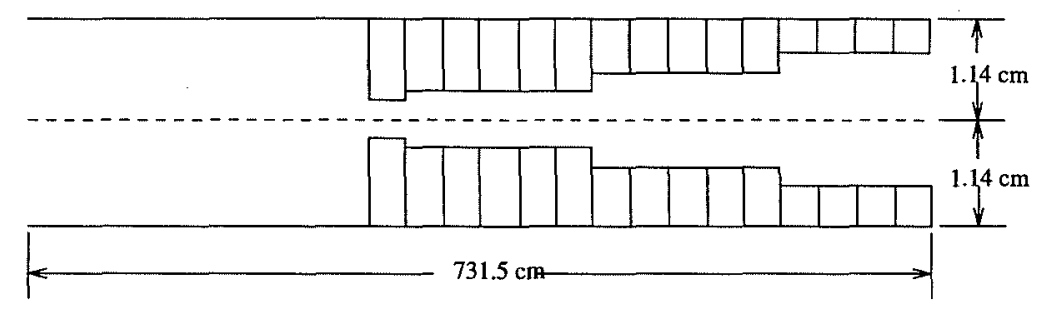

Brass

Figure 2.3: The charged collimator in PC3ANA. 


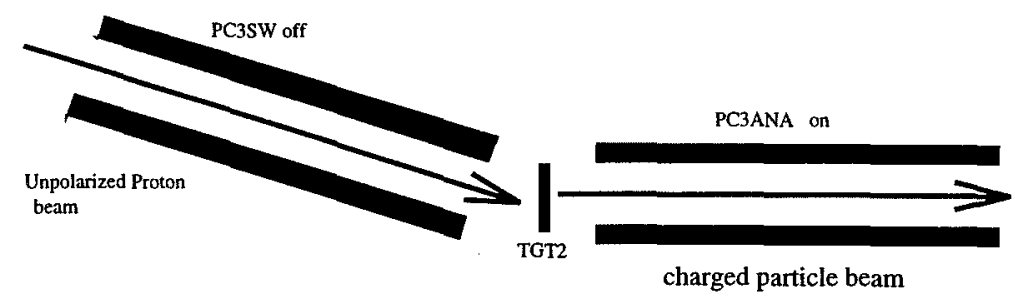

Figure 2.4: The proton production mode.

the SWICs and the SEM provided a means of monitoring the intensity and quality of the proton beam on a spill by spill basis.

\subsection{Production Methods}

In this experiment there were three different production modes: proton production, unpolarized neutral beam production, and polarized neutral beam production. Figure 2.4 shows how the magnets and targets were arranged for the proton production mode. The upstream target, PC3TGT1 was removed from the beamline, and dipole magnet PC3SW was turned off. In addition, PC3SW was aligned so that the proton beam could pass through the collimator and strike the second target, PC3TGT2, at the desired production angle.

In the unpolarized neutral beam production mode, shown in Figure 2.5, the upstream target, PC3TGT1 was placed in the beam. The protons struck this target at an angle of $0 \mathrm{mr}$. The particles produced at this target then passed through PC3SW where charged particles were bent out of the beamline. This resulted in a beam of unpolarized neutral particles which then struck the downstream target. As in the proton production modes, PC3SW could be raised or lowered to achieve the desired production angle. Data was taken using this production method at two different settings of the current in charged sweeper magnet, PC3ANA, to take data with a wider range of momentum values.

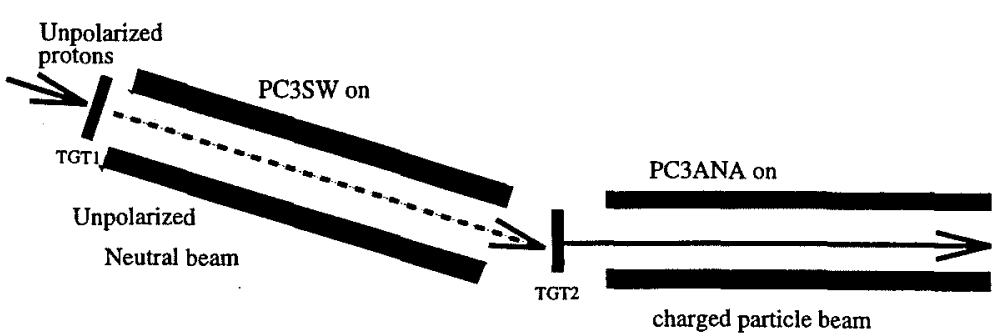

Figure 2.5: The unpolarized neutral beam production mode.

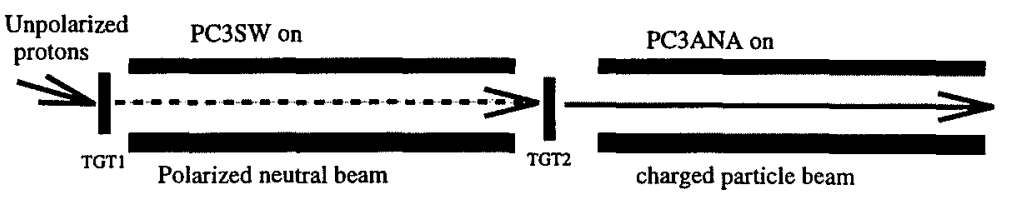

Figure 2.6: The polarized neutral beam production mode.

The polarized neutral beam production mode, shown in Figure 2.6, was similar to the unpolarized neutral beam production mode except that PC3SW always remained horizontal. The protons struck the upstream target at an angle determined by the fields in PC3V1 and PC3V2. The particles produced then entered PC3SW, where the charged particles were bent out of the beamline. The beam that exited PC3SW contained polarized neutral particles which then struck the downstream target at an angle of 0 mrad.

Data was also taken with both production angles at 0 mrad. This was done to produce $\Xi^{-} \mathrm{s}$ and $\Omega^{-} \mathrm{s}$ that were known to be unpolarized for use in systematic studies during the polarization analysis. This production mode is similar to that shown in Figure 2.6, except that the protons were incident on PC3TGT1 at 0 mrad. 


\subsection{Coordinate System}

In the spectrometer, positive $z$-was defined as the direction tangent to the central orbit of the charged collimator at its downstream exit. Positive $y$ was defined to be up, and $x$ was defined such that the coordinate system was right-handed as shown in Figure 2.7. The origin of this coordinate system was based at the exit of the Hyperon magnet collimator. The sign of the production angle was defined by the sign of $\vec{P}_{b e a m} \times \vec{p}_{\Xi-}$ in a right handed coordinate system with its origin at the downstream target.

\subsection{The Spectrometer}

After exiting the Hyperon magnet collimator, the particles entered the spectrometer, which was designed to detect the three-track decays $\Omega^{-} \rightarrow \Lambda+K^{-}$and $\Xi^{-} \rightarrow \Lambda+\pi^{-}$ each with a subsequent $\Lambda \rightarrow p+\pi^{-}$decay. The spectrometer consisted of four planes of silicon strip detectors (SSDs) in each view ( $x$ and $y$ ) to track the parent hyperon or its charged daughter, several scintillation counters to provide fast trigger signals, and twelve multiwire proportional chambers (MWPCs) to provide tracking information about the decay products. Figure 2.7 shows the spectrometer, and Table 2.1 lists all the elements of the spectrometer and gives their sizes and positions relative to the exit of the Hyperon magnet. Two dipole analyzing magnets were used to determine the momentum of the daughter protons, pions and kaons. Helium filled bags and tubes were placed between the MWPCs to reduce multiple scattering of the daughter particles. The total amount of material in the beamline was $9.55 \times 10^{-2}$ interaction lengths.

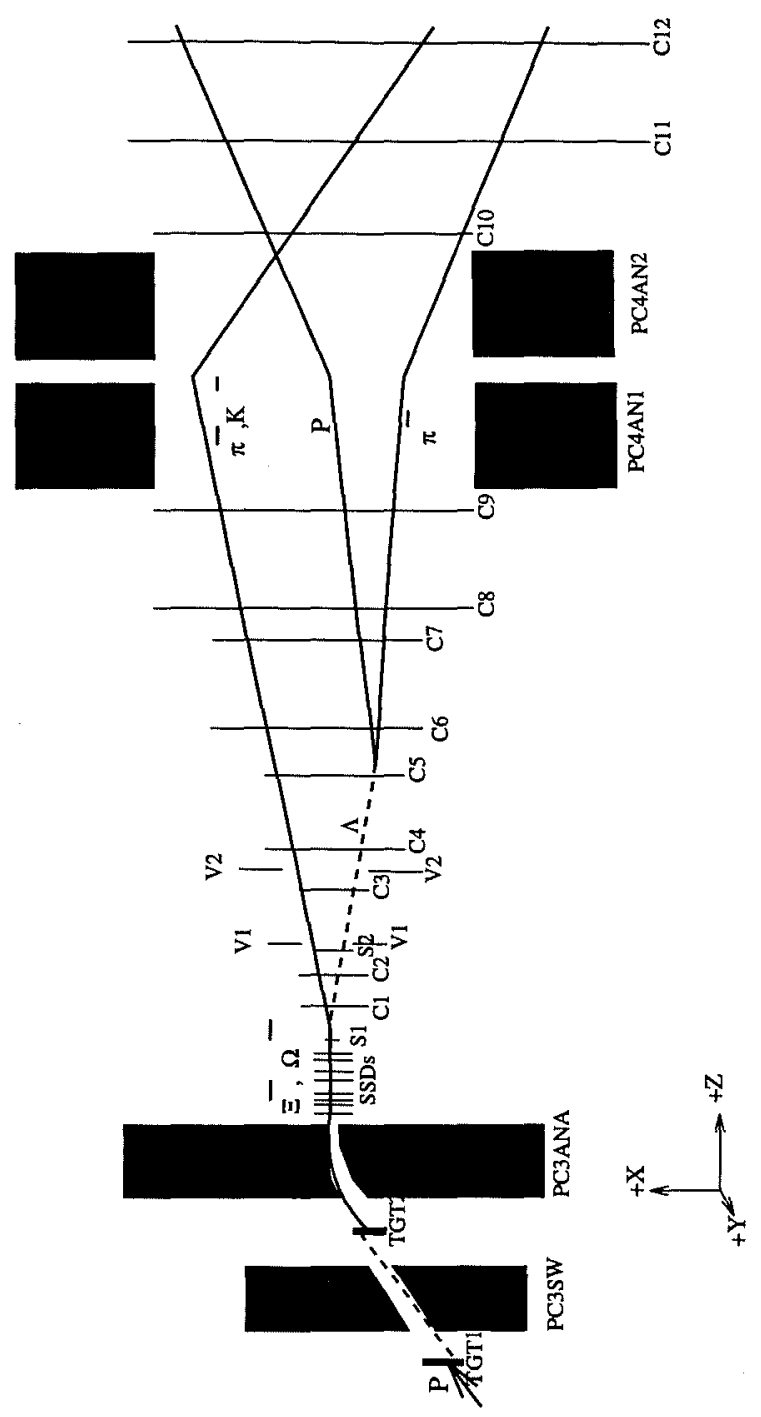

Figure 2.7: Plan view of the E800 spectrometer (not to scale). Also shown is a typical $\Xi^{-}$decay. 


\begin{tabular}{c|c|c|c|c|c|c} 
Detector & Position & Width & Height & Thickness & Pitch & Device Type \\
\hline SSD1(x) & 74.43 & 2.8 & 2.8 & 0.03 & .01 & SSD \\
SSD1(y) & 79.22 & 2.8 & 2.8 & 0.03 & .01 & SSD \\
SSD2(x) & 100.97 & 2.8 & 2.8 & 0.03 & .01 & SSD \\
SSD2(y) & 109.97 & 2.8 & 2.8 & 0.03 & .01 & SSD \\
SSD3(x) & 129.46 & 2.8 & 2.8 & 0.03 & .01 & SSD \\
SSD3(y) & 137.80 & 2.8 & 2.8 & 0.03 & .01 & SSD \\
SSD4(x) & 158.43 & 2.8 & 2.8 & 0.03 & .01 & SSD \\
SSD4(y) & 166.29 & 2.8 & 2.8 & 0.03 & .01 & SSD \\
S1 & 360. & 6.35 & 3.81 & .1 & n/a & scintillator \\
C1(x,y) & 561. & 12.8 & 12.8 & n/a & 1.0 & MWPC \\
C2(x,y) & 776. & 12.8 & 12.8 & n/a & 1.0 & MWPC \\
V1 & 800. & 32.38 & 8.89 & .32 & n/a & scintillator \\
V1(hole $)$ & 800. & 11.43 & 6.35 & .32 & n/a & n/a \\
S2 & 800. & 10.79 & 6.35 & .1 & n/a & scintillator \\
C3(x,y) & 988. & 12.8 & 12.8 & n/a & 1.0 & MWPC \\
V2 & 1020. & 41.91 & 11.43 & .32 & n/a & scintillator \\
V2(hole) & 1020. & 13.97 & 8.25 & .32 & n/a & n/a \\
C4(x,y) & 1511. & 25.6 & 25.6 & n/a & 1.0 & MWPC \\
C5(x,y) & 2009. & 25.6 & 25.6 & n/a & 1.0 & MWPC \\
C6(x,y) & 2499. & 51.2 & 25.6 & n/a & 2.0 & MWPC \\
C7(u,v) & 3013. & 12.8 & 12.8 & n/a & 2.0 & MWPC \\
C8(u,v) & 3089. & 51.2 & 51.2 & n/a & 2.0 & MWPC \\
C9(x,y,u) & 3697. & 51.2 & 51.2 & n/a & 2.0 & MWPC \\
C10(x,y) & 4261. & 63.8 & 25.6 & n/a & 2.0 & MWPC \\
C11(x,y) & 4840. & 128. & 38.4 & n/a & 2.0 & MWPC \\
C12(x,y) & 6154. & 128. & 38.4 & n/a & 2.0 & MWPC \\
DW. & & & & & & \\
1. & & & &
\end{tabular}

Table 2.1: The size and $z$ positions of the spectrometer elements. All measurements are given in centimeters. Width and height refer to the active area of the detector. (n/a not applicable)

\subsubsection{The Silicon Strip Detectors}

The SSDs were the most upstream element of the spectrometer. They were used to accurately track the parent $\Xi^{-} s$ and $\Omega^{-} s$ before they decayed and also aided in differentiating between the negatively charged daughter from the parent decay and the negatively charged daughter from the $\Lambda^{0}$ decay. There were eight planes of SSDs (manufactured by Hammamatsu) with $0.3 \mathrm{~mm}$ thickness and 280 strips of $100 \mu \mathrm{m}$ pitch. Laben Model MSP1 pre-amplifiers were used to amplify the charge (on the order of 1 fC) collected on a strip when a charged particle passed through it. These pre-amps gave a $1.0-2.0 \mathrm{mV}$ output that was amplified and discriminated by Nanometrics Model N-277CD 16 channel amplifier cards. The outputs of the discriminators went into Nanometrics Model N-278 data latches which were read out using the Nanometric Model N-281 CAMAC read-out system. The sensitivity of the MSP1 preamps made them very susceptible to noise, so substantial effort was made to shield data cables and insure that the system was properly grounded. In addition, considerable effort was expended to insure that all components of the Nanometric read-out system functioned properly.

Table 2.2 gives the efficiency of each of the SSD planes for one run. These efficiencies were measured using single track events. To calculate the efficiency of a plane, a track was fit without using that plane, and then the hits in that plane were examined to determine if any of them were within a distance equal to 1.5 times the pitch of the detector from the fit track. The efficiency was defined to be the percentage of the total events with a hit within 1.5 times the pitch of the fit track. In practice, the separation of hits was always quantized by the pitch of the detector plane. 


\begin{tabular}{c|c} 
SSD & Efficiency (\%) \\
\hline SSD1(x) & 83. \\
SSD1(y) & 63. \\
SSD2(x) & 82. \\
SSD2(y) & 84. \\
SSD3(x) & 81. \\
SSD3(y) & 85. \\
SSD4(x) & 84. \\
SSD4(y) & 82.
\end{tabular}

Table 2.2: SSD Efficiencies

\subsubsection{The Scintillation Counters}

The set of four scintillation counters, S1, V1, S2, and V2 were used to define the beam. The dimensions of these counters are given in Table 2.1. Counters S1 and S2 were used to identify charged particles that were within the beam defined by the hyperon magnet collimator. The veto counters, V1 and V2, had holes in them. The presence of a particle outside the hole region indicated a charged particle that was not from the negative beam region. Counters $\mathrm{S} 1$ and $\mathrm{S} 2$ had one photomultiplier tube, but because of their center holes, counters V1 and V2 had two photomultiplier tubes, one on either side of the hole. The signals from these two phototubes were logically ORed together to produce one signal for each counter.

The signals from the scintillation counters were discriminated by standard NIM discriminators whose outputs were used in the trigger and were also input to scalers that were read-out at the end of each spill.

\subsubsection{The Multiwire Proportional Chambers}

Twelve MWPCs were used in the spectrometer. With the exception of $\mathrm{C} 7$ and $\mathrm{C} 8$, all MWPCs had sense planes in the $\hat{x}$ and $\hat{y}$ directions. The sense planes in $\mathrm{C} 7$ and $\mathrm{C} 8$ were rotated 45 degrees with respect to the sense planes of the other chambers. These rotated planes are referred to as $\hat{u}$ and $\hat{v}$ planes and were used in the track finding to match the $x$ and $y$ tracks for each particle. Also, in addition to its $x$ and $y$ planes, C9 had a $u$ sense plane with a 45 degree rotation.

Each MWPC except C9 consisted of three high voltage planes which sandwiched the two sense planes. C9 had four high voltage planes and three sense planes. The sense wires in $\mathrm{C} 1, \mathrm{C} 2, \mathrm{C} 3, \mathrm{C} 4$, and $\mathrm{C} 5$ had a wire spacing of $1 \mathrm{~mm}$ and the other chambers had $2 \mathrm{~mm}$ pitch sense planes. Gold plated tungsten wire was used in the sense planes. The high voltage planes in $\mathrm{C} 1, \mathrm{C} 2, \mathrm{C} 3$, and $\mathrm{C} 5$ were made with 0.25 mil thick aluminized mylar foils, and the high voltage planes in the other chambers consisted of 2 or 3 mil copper-beryllium wire at $1 \mathrm{~mm}$ spacing. $\mathrm{C} 4$ and $\mathrm{C} 5$ experienced many stability problems during the run, and during the last part of the run, the $y$ sense plane of $\mathrm{C} 5$ was disconnected due to excessive sparking.

The gas used in all of the chambers was a mixture of $95 \%$ argon, $5 \%$ methylal, and $0.12 \%$ freon where the percentages are given by gas volume. The correct fraction of methylal was maintained by bubbling the argon - freon mixture through liquid methylal at 2 degrees Celsius. The chamber efficiencies are given in Table 2.3, and were measured using the same method used to measure the SSD efficiencies.

The read-out system for C1, C2, and C3 used LeCroy amplifier-discriminator cards that fed into Nanometric Model N-278 latches. The latches were read-out using the same system used for the SSD hit data. The read-out system for the remaining MWPCs utilized custom-built electronics. This system consisted of four channel amplifierdiscriminator-latch cards from which data was gathered using a "sparse read-out" system[38]. The amplifier cards used in the MWPCs upstream of the analyzing magnets were more sensitive since these chambers were operated at a lower high voltage, which was desirable because of the high particle fluxes in this portion of the spectrometer. In addition, the amplifier cards used in $\mathrm{C} 4$ and $\mathrm{C} 5$ had their discriminator thresholds lowered so that these chambers could be operated at even lower high voltages. This was done in an effort to improve the efficiency of these detectors, however, their operation 


\begin{tabular}{c|c} 
Chamber & Efficiency (\%) \\
\hline $\mathrm{C} 1(\mathrm{x})$ & 94. \\
$\mathrm{C} 1(\mathrm{y})$ & 93. \\
$\mathrm{C} 2(\mathrm{x})$ & 92. \\
$\mathrm{C} 2(\mathrm{y})$ & 94. \\
$\mathrm{C} 3(\mathrm{x})$ & 95. \\
$\mathrm{C}(\mathrm{y})$ & 95. \\
$\mathrm{C} 4(\mathrm{x})$ & 69. \\
$\mathrm{C} 4(\mathrm{y})$ & 67. \\
$\mathrm{C} 5(\mathrm{x})$ & 93. \\
$\mathrm{C} 5(\mathrm{y})$ & 94. \\
$\mathrm{C} 6(\mathrm{x})$ & 98. \\
$\mathrm{C} 6(\mathrm{y})$ & 99. \\
$\mathrm{C} 7(\mathrm{u})$ & 94. \\
$\mathrm{C} 7(\mathrm{v})$ & 98. \\
$\mathrm{C} 8(\mathrm{u})$ & 96. \\
$\mathrm{C} 8(\mathrm{v})$ & 98. \\
$\mathrm{C} 9(\mathrm{x})$ & 96. \\
$\mathrm{C} 9(\mathrm{y})$ & 97. \\
$\mathrm{C} 10(\mathrm{x})$ & 98. \\
$\mathrm{C} 10(\mathrm{y})$ & 100. \\
$\mathrm{C} 11(\mathrm{x})$ & 95. \\
$\mathrm{C} 11(\mathrm{y})$ & 97. \\
$\mathrm{C} 12(\mathrm{x})$ & 96. \\
$\mathrm{C} 12(\mathrm{y})$ & 97.
\end{tabular}

Table 2.3: MWPC Efficiencies.

was never totally satisfactory.

\subsubsection{The Analyzing Magnets}

The magnets between $\mathrm{C} 9$ and $\mathrm{C} 10$ were used to momentum analyze the charged particles. These magnets were two BM109 dipoles. Both magnets had a $x$ aperture of 61.0 $\mathrm{cm}$. The upstream magnet had a $y$ aperture of $25.4 \mathrm{~cm}$ and the downstream magnet had a $y$ aperture of $30.5 \mathrm{~cm}$. Each magnet was $182 . \mathrm{cm}$ long and they were separated by a distance of $56 . \mathrm{cm}$. Both magnets had mirror planes to reduce the fringe fields.

The magnetic field of these magnets was determined using two different methods.
First, the "Ziptrack" apparatus was used to make three dimensional field maps of the two magnets. This apparatus was built at Fermilab and uses Hall probes to measure the three magnetic field components at various points inside and outside of the magnet. This apparatus was used to map the field inside the magnet and the fringe field just outside of the magnets. These field maps were used to search for non-homogeneity in the magnets. The fields in both magnets were found to be constant to within a few percent. The second method used the reconstructed masses of the $\Xi^{-} \mathrm{s}$ and $\Lambda \mathrm{s}$ to determine the $p_{T}$ kick of the magnets. This value was found to be $-1.445 \mathrm{GeV} / \mathrm{c}$ at the standard operating current.

\subsection{Trigger}

The trigger used to detect three-track events consisted of two parts. The first part used scintillation counters S1, S2, V1, and V2. This trigger required hits in S1 and S2 in conjunction with no hits in $\mathrm{V} 1$ and $\mathrm{V} 2$ (i.e. $S 1 \cdot S 2 \cdot \overline{V 1} \cdot \overline{V 2}$ ). This insured that a charged particle had entered the spectrometer from the hyperon beam and was not part of a shower from an upstream interaction. This part of the trigger, referred to as the pi trigger, was a very effective way of selecting single track particles which were useful in aligning the spectrometer, monitoring detector efficiency and other systematic studies. For these reasons, pi triggers were written to most of the data tapes at rates that were prescaled to be between $1 / 32$ and $1 / 1024$ of the three-track trigger rate.

The characteristic "V" topology of the $\Lambda$ that was a daughter of both the $\Xi^{-}$and $\Omega^{-}$ decays was used to make a trigger for three-track events. This was done by triggering on charged particles passing through the side of $\mathrm{C} 11$ hit by negative pions from the decays and the side of $\mathrm{C} 12$ hit by protons from the decays. The complete three track trigger was:

$$
S 1 \cdot S 2 \cdot \overline{V 1} \cdot \overline{V 2} \cdot(C 11)_{\pi} \cdot(C 12)_{p r o t o n}
$$


This was the simplest possible trigger that would still allow us to take data with a reasonable live time (greater that $60 \%$ ), given the limitations of the readout electronics. The live time is simply the percentage of triggers that were actually readout. A simpler trigger also reduced the possibility of systematic errors due to the trigger. Studies of possible biases due to the trigger are discussed in Chapter 5 .

\subsection{Data Acquisition System and Experimental Opera-}

\section{tion}

The main consideration during the design of the data acquisition system was maximizing the amount of data that could be collected during one spill. Since the most time consuming process was writing the data to tape, a system in which the data was stored in fast semiconductor memory during the spill and then written to tape during the time between spills was adopted. Figure 2.8 shows a schematic of the data acquisition system.

All of the detectors were read out using a CAMAC system interfaced to a VAXstation Model 3200. When a trigger occurred, a signal was sent to the electronics to prepare for read-out. The trigger also went to a Fermilab designed CAMAC Smart Crate Controller (SCC)[39]. This SCC was designed to execute pre-loaded lists of CAMAC instructions at a rate of $1 \mathrm{MHz}$. When the $\mathrm{SCC}$ received a trigger signal, it executed a list of instructions that read out the data from the SSDs, MWPCs, and various other latches and scalers. This data was written into a fast first-in, first-out (FIFO) memory unit. At the end of each spill, scalers that recorded trigger element hits, the SEM, and other counters were read out. Then, the data in the FIFO was transferred to the VAXstation and written to $8 \mathrm{~mm}$ video tape. Each data tape held an average of ten data runs, with each run containing about 500,000 events, and each event containing approximately 200 bytes of data. Table 2.4 gives a summary of the

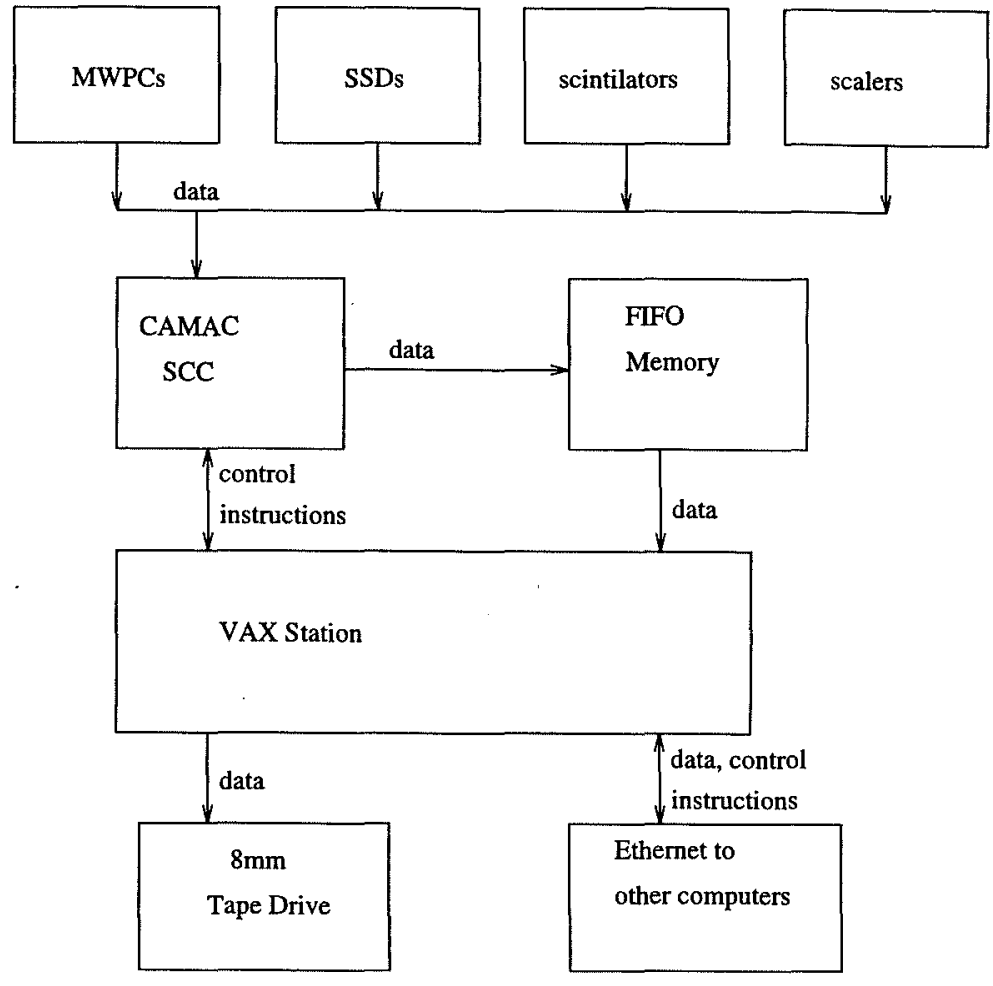

Figure 2.8: The data acquisition system. 


\begin{tabular}{l|c|c|c} 
Production Mode & Angle & Runs & $\begin{array}{c}\text { Three-track } \\
\text { Triggers }\end{array}$ \\
\hline Unpolarized Neutral Beam & + & 711 & $346,982,603$ \\
\cline { 2 - 4 } Hyperon Magnet =-2900 & - & 757 & $374,297,889$ \\
\hline Unpolarized Neutral Beam & + & 308 & $150,467,562$ \\
\cline { 2 - 4 } Hyperon Magnet =-750 & - & 289 & $142,700,032$ \\
\hline Polarized Neutral Beam & + & 182 & $87,777,817$ \\
\cline { 2 - 4 } & - & 171 & $84,086,479$ \\
\hline Zero Mrad & + & 170 & $82,531,343$ \\
\cline { 2 - 4 } & - & 172 & $84,187,513$ \\
\hline \hline Totals & & 2760 & $1,353,031,238$ \\
\hline
\end{tabular}

Table 2.4: Summary of data collected. position. Magnet currents were monitored and controlled using the Fermilab Epicure beamline control system. The magnet currents and SWIC positions were recorded in the experimental logbooks several times during each data tape.

number of data runs and triggers written to tape for each of the various production modes and angles reported on in this thesis. The run size was selected to produce data files that were of a manageable size, and to take approximately 30 minutes, which was thought to be a reasonable interval between checks of the beam positions and other experimental conditions. Under normal operating conditions, each unpolarized neutral beam production run took approximately 20 minutes. Polarized neutral beam runs took approximately 40 minutes due to lower particle production rates. The main factors limiting the data taking rate were radiation loss restrictions in the targeting area and deadtime limitations of the spectrometer. The live time of the experiment was always greater than $60 \%$.

Some fraction of the data that was written to tape was also distributed via ethernet to other computers. These computers performed a quick analysis of the events to monitor the performance of the spectrometer and data acquisition system. The amount of data available for this monitoring was limited by several factors, including network loading, but was usually at least $50 \%$.

During the data runs, the production angle was monitored by observing the beam positions on the SWICs. Also, the number of one track and three track triggers per delivered proton were monitored as an additional check of the spectrometer and beam 

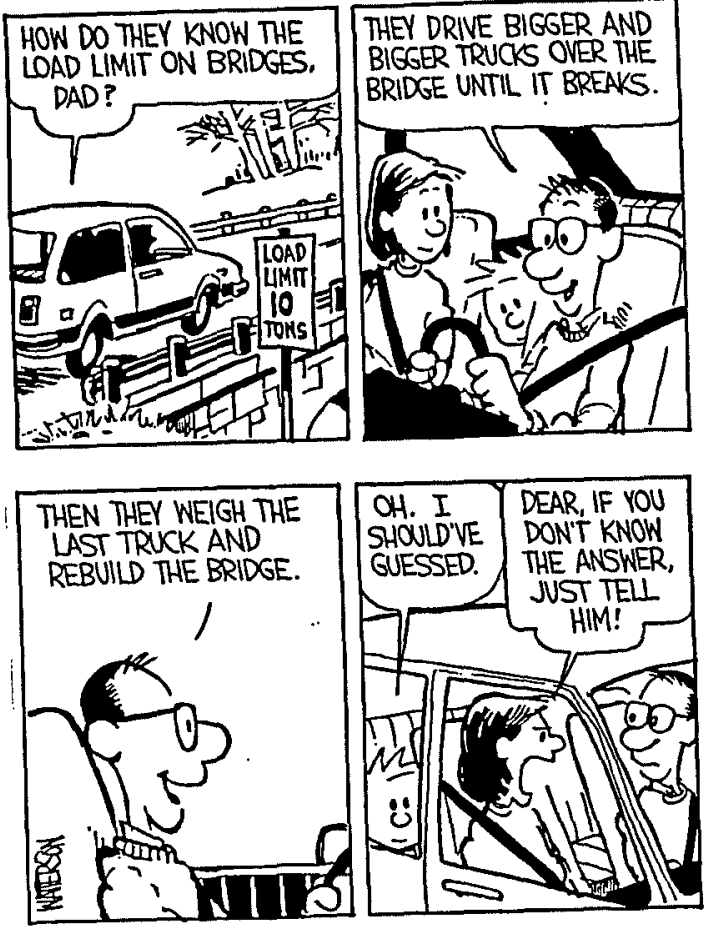

CALVIN AND HOBBES copyright Watterson. Dist. by UNIVERSAL PRESS SYNDICATE. Reprinted with permission. All rights reserved.

\section{Chapter 3}

\section{Event Reconstruction and}

\section{Selection}

\subsection{Introduction}

The three charged track topology of the decays $\Xi^{-} \rightarrow \Lambda+\pi^{-}$or $\Omega^{-} \rightarrow \Lambda+K^{-}$, followed by $\Lambda \rightarrow p+\pi^{-}$allowed the events to be completely reconstructed using only the tracking information from the hits in the MWPCs. The SSD information added additional constraints on the parent track when available. The three tracks in the $x$ view were bent by the momentum analyzing magnet, with the proton, which is the highest momentum track, being bent in the opposite direction from the two negatively charged tracks. Once the three daughter tracks had beer reconstructed, they were fit to a $\Xi^{-}$or $\Omega^{-}$hypothesis using geometric and kinematic constraints.

\subsection{The First Pass Analysis}

An initial pass was made through the raw data tapes to eliminate events which did not have enough information to be successfully reconstructed as three-track events. 
Description of Selection Criteria

\begin{tabular}{l|c} 
& $\begin{array}{c}\text { Three-track } \\
\text { Triggers Eliminated }\end{array}$ \\
\hline Less than 2 hits in 3 planes of C6 and C8 & 62.5 \\
Less than 3 hits in C7 and C8 and less than 2 hits in C9u & 0.0 \\
Less than 2 hits in 2 $x$-planes of C10, C11, or C12 & 2.8 \\
Less than 2 hits in 3y-planes of & 5.5 \\
C6, C7, C8, C9, C10, C11, or C12 &
\end{tabular}

Table 3.1: Selection criteria for first pass reconstruction. These percentages are for a typical unpolarized neutral beam production data run.

\begin{tabular}{l|c|c|c|c} 
Production Mode & Angle & Runs & $\begin{array}{c}\text { Three-track } \\
\text { Triggers }\end{array}$ & $\begin{array}{c}\text { Three-track } \\
\text { Candidates }\end{array}$ \\
\hline Unpolarized Neutral Beam & + & 711 & $346,982,603$ & $102,177,861$ \\
\cline { 2 - 5 } Hyperon Magnet = -2900 & - & 757 & $374,297,889$ & $110,232,099$ \\
\hline Unpolarized Neutral Beam & + & 308 & $150,467,562$ & $46,929,723$ \\
\cline { 2 - 5 } Hyperon Magnet = -750 & - & 289 & $142,700,032$ & $45,346,166$ \\
\hline Polarized Neutral Beam & + & 182 & $87,777,817$ & $34,212,605$ \\
\cline { 2 - 5 } & - & 171 & 84086,479 & $30,240,120$ \\
\hline Zero Mrad & + & 170 & $82,531,343$ & $38,000,350$ \\
\cline { 2 - 5 } & - & 172 & $84,187,513$ & $39,097,574$ \\
\hline \hline Totals & & 2760 & $1,353,031,238$ & $446,236,498$ \\
\hline
\end{tabular}

Table 3.2: Summary of data remaining after first pass.

Table 3.1 shows the criteria used and the percentage of events eliminated by each selection criteria.

The raw data from events that passed these selection criteria were written to first level summary tapes. Table 3.2 shows the number of three-track events remaining for each data taking mode after this step. This pass removed $67 \%$ of the raw triggers from the three-track data sample. In addition, events that passed the criteria of a good single track event were written to a separate set of tapes for use in calibrating and aligning the experiment.

\subsection{The Reconstruction Program}

The reconstruction program was a multi-step process starting from the first level summary data described above. Figure 3.1 shows a schematic diagram of the overall reconstruction process. In this experiment it was very important that the reconstruction program mis-reconstruct as few events as possible since any incorrectly reconstructed event could have a noticeable effect on the measured asymmetries. In addition it was important that the efficiency of the reconstruction process not vary appreciably over the range of selected events. To do this, three different reconstruction methods were used. The different methods each had their strengths and weaknesses, but by combining the three methods, their weaknesses could be minimized.

The first stage of the reconstruction process involved additional hit counting criteria to remove events that Monte Carlo studies showed were difficult to reconstruct correctly. These selection criteria and the corresponding percentage of events each eliminated are given in Table 3.3 .

\begin{tabular}{l|c}
$\begin{array}{l}\text { Description of } \\
\text { Selection Criteria }\end{array}$ & $\begin{array}{c}\text { Percentage of First } \\
\text { Level Summary } \\
\text { Events Eliminated }\end{array}$ \\
\hline Two or more hits in the $y$ views of C6, C9, and C10 & 4.2 \\
Two or more hits in the $x$ views of C6, C9, C10, and C11 & 23.1 \\
Fewer than 3 planes of 6 - C12 have more than four hits & 28.1
\end{tabular}

Table 3.3: Hit counting data selection criteria. These percentages are for a typical unpolarized neutral beam production data run.

\subsubsection{Initial Reconstruction Program}

Any event that survived these hit counting criteria was passed to the basic three-track reconstruction program. This program began by looking at the hits in five of the $2 \mathrm{~mm}$ chambers $(\mathrm{C} 6, \mathrm{C} 9, \mathrm{C} 10, \mathrm{C} 11, \mathrm{C} 12)$. The $y$ tracks were found first since they were straight while the $x$ tracks were bent by the analysis magnets. If three $y$ tracks were found, the 


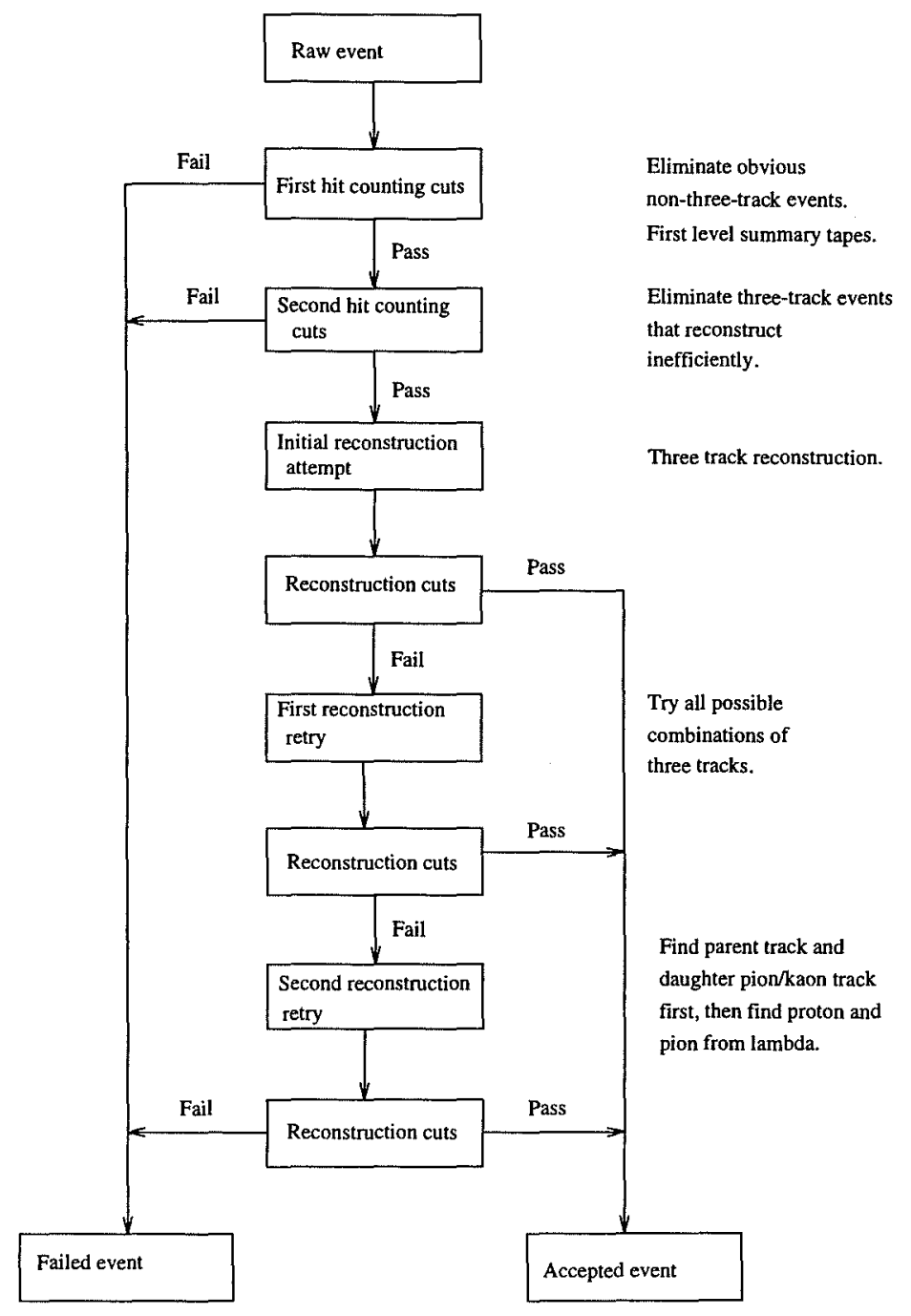

Figure 3.1: The reconstruction process. program next looked for three tracks in the $x$ view of the $2 \mathrm{~mm}$ chambers upstream of the analysis magnets. Then, the $x$ and $y$ tracks were matched using the information from the $u$ and $v$ planes of $\mathrm{C}$, C8, and C9. Finally, three $x$ tracks were found in the chambers downstream of the analysis magnets and matched with the upstream tracks at the magnet bendplane.

Next, an attempt was made to find the parent track in the SSDs, and then hits from the $1 \mathrm{~mm}$ chambers $(\mathrm{C} 1, \mathrm{C} 2, \mathrm{C} 3, \mathrm{C} 4$, and $\mathrm{C} 5)$ were added to the parent and daughter tracks. Accepted events were not required to have a parent track, however, the parent track did help reduce the uncertainty in the parent vertex measurement. Then, the daughter tracks for any successful solutions were fit to a three-track topology in both views simultaneously. The fitting routine calculated the location of the $\Xi^{-}$or $\Omega^{-}$and $\Lambda$ vertices, the momentum vectors of all the particles involved in the decay, the position of the parent particle at the target, $X_{T}$ and $Y_{T}$, and the mass of the parent particle using both a $\Xi^{-}$and $\Omega^{-}$hypothesis. In addition, the fitting routine calculated a geometric chi-squared per degree of freedom, $\chi_{G}^{2} / D F$, which was used to judge how well the event was fit.

The final part of this procedure varied the proton and $\pi$ momentum vectors within their uncertainties to force the $\Lambda$ to have the correct mass. This procedure calculated a parameter, $\chi^{2}{ }_{\Lambda}$, which was a measure of the difference between the $\Lambda$ solution for the three-track, two-vertex fit and the solution with the correct mass, and was used as another data selection criteria.

All events that were successfully reconstructed by this procedure were required to pass a set of data selection criteria that were intended to remove background and badly reconstructed events. These data selection criteria are listed in Table 3.4.

Any events that passed these selection criteria were accepted as correctly reconstructed events. Of the final unpolarized neutral beam production events used in the 


\begin{tabular}{l|c|c} 
Description of Data Selection Criteria & $\begin{array}{c}\text { Real Events } \\
\text { Removed }\end{array}$ & $\begin{array}{c}\text { Monte Carlo } \\
\text { Removed }\end{array}$ \\
\hline mass within $15 \mathrm{MeV}$ of $m_{\Xi-}$ or $m_{\Omega^{-}}$ & $40.8 \%$ & $33.5 \%$ \\
\hline$\chi_{G}^{2} / D F<4$ & $2.9 \%$ & $4.0 \%$ \\
\hline$\chi_{\Lambda}^{2}<15$ & $5.0 \%$ & $5.3 \%$ \\
\hline$\left|X_{T}\right|<0.9 \mathrm{~cm}$ & $2.0 \%$ & $1.8 \%$ \\
$\left|Y_{T}\right|<0.8 \mathrm{~cm}$ & & \\
\hline no hits used on more than one track & & \\
$\begin{array}{l}\text { at least 2 hits used in } \mathrm{C} 6, \mathrm{C} 9, \mathrm{C} 10, \text { and } \mathrm{C} 11 x \\
\text { at least } 2 \text { hits used in } \mathrm{C} 6, \mathrm{C} 9, \text { and } \mathrm{C} 10 y\end{array}$ & $45.7 \%$ & $46.3 \%$
\end{tabular}

Table 3.4: Reconstructed data selection criteria.

polarization analysis, $67.3 \%$ were accepted by this reconstruction pass. When Monte Carlo data was analyzed, this reconstruction pass contributed $70.5 \%$ of the accepted events.

\subsubsection{First Reconstruction Retry}

All events which passed the initial hit counting criteria, but could not be successfully reconstructed by the initial reconstruction program were reprocessed using a different track finding method.

This track finding method first found all possible tracks in the $y$ views of $\mathrm{C} 6, \mathrm{C} 8$, and C10. Any track would not lead to a valid solution were not considered. For example, tracks that pointed outside the analysis magnet apertures were eliminated. From the tracks that remained, sets of three tracks were selected as possible tracks from the $\Xi^{-}$ or $\Omega^{-}$decay. The two tracks assigned as the $\Lambda$ daughter particles were required to have a vertex upstream of $\mathrm{C} 6$. Next, all possible upstream and downstream $x$ tracks were constructed. Any upstream and downstream $x$ tracks which intersected the bendplane of the analysis magnets within $0.5 \mathrm{~cm}$ of each other were joined, and then the $x$ and $y$ tracks were matched using hits from the $u$ and $v$ planes of $\mathrm{C} 7, \mathrm{C} 8$, and $\mathrm{C} 9$.

All possible combinations of three matched $x$ and $y$ tracks were made and each of these sets of solutions was then passed through a series of fitting routines that eliminated solutions with incorrect hit assignments. The first fitting routine required that the two tracks that were thought to correspond to the $\Lambda$ daughter particles have a good geometric fit to the $\Lambda$ topology. This fitting routine was able to reject incorrect solutions quickly because the algebra required to fit the $\Lambda$ topology was exactly soluble.

Solutions that survived these fitting routines were then passed to the geometric threetrack fitter described in section 3.3.1. Solutions with values of $\chi_{G}^{2} / D F<10$ were then passed to the kinematic $\Lambda$ fitter used in the initial reconstruction pass (section 3.3.1), and solutions with $\chi^{2}{ }_{\Lambda}>100$ were eliminated.

Next, an attempt was made to add additional hits from the $1 \mathrm{~mm}$ chambers, and then the geometric three-track fit was redone. The solution was then required to pass the data selection criteria in Table 3.4. If more than one possible solution remained, the solution with the smallest $\chi_{G}^{2} / D F$ was found and any solution with $\chi_{G}^{2} / D F$ more than 0.2 above this minimum was rejected. From the remaining solutions, the one with the lowest $\chi^{2}{ }_{\Lambda}$ was selected. The portion of the final unpolarized neutral beam data sample reconstructed by this retry method was $19.5 \%$. When analyzing Monte Carlo data, $15.3 \%$ of the accepted sample came from this retry process.

\subsubsection{Second Retry Method}

At this point in the reconstruction process, any event that had not been successfully reconstructed by either the initial reconstruction program or the first retry was sent to a second and final retry program. This program used a different approach to the problem of correctly assigning the hits to the various tracks. Instead of trying to find the tracks corresponding to the daughter particles first, this approach first looked for the parent track in the SSDs and the most upstream 1mm chambers. It then used the knowledge that one of the daughter tracks would originate at the $\Xi^{-}$or $\Omega^{-}$vertex and 


\begin{tabular}{l|c|c|c|c} 
Production Mode & Angle & $\begin{array}{c}\text { Three-track } \\
\text { Candidates }\end{array}$ & $\begin{array}{c}\Xi^{-} \\
\text {Candidates }\end{array}$ & $\begin{array}{c}\Omega^{-} \\
\text {Candidates }\end{array}$ \\
\hline Unpolarized Neutral Beam & + & $102,177,861$ & $14,474,537$ & $1,822,051$ \\
Hypperon Magnet = -2900 & - & $110,232,099$ & $14,886,637$ & $1,873,727$ \\
\hline Unpolarized Neutral Beam & + & $46,929,723$ & $5,510,850$ & 765,328 \\
\cline { 2 - 5 } Hyperon Magnet = -750 & - & $45,346,166$ & $5,238,496$ & 726,001 \\
\hline Polarized Neutral Beam & + & $34,212,605$ & $1,201,471$ & 155,917 \\
\cline { 2 - 5 } & - & $30,340,120$ & $1,275,716$ & 161,010 \\
\hline Zero Mrad & + & $38,000,350$ & $1,818,638$ & 223,249 \\
\cline { 2 - 5 } & - & $39,097,574$ & $1,875,620$ & 230,205 \\
\hline \hline Totals & & $446,236,498$ & $46,281,965$ & $5,957,488$ \\
\hline
\end{tabular}

Table 3.5: Summary of data remaining after the reconstruction pass.

therefore should intersect the parent track. Typically, one or two possible solutions for the daughter $K^{-}\left(\pi^{-}\right)$from the $\Omega^{-}\left(\Xi^{-}\right)$were found, and for each solution, the remaining hits were used in an attempt to fit a $\Lambda$.

When a good $\Lambda$ solution was found, the event was passed through the geometric and kinematic fitters described in section 3.3.1. Next, the data selection criteria from Table 3.4 were imposed. If more than one good solution for an event was found, the best solution was selected using the same method utilized in section 3.3.2. This retry contributed $13.2 \%$ of the events in the reconstructed unpolarized neutral beam data sample. When Monte Carlo data was analyzed, this retry contributed $14.2 \%$ of the accepted sample.

Table 3.5 shows the total number of reconstructed events that were accepted under both the $\Xi^{-}$and $\Omega^{-}$mass hypothesis.

\subsection{The Monte Carlo Program}

A Monte Carlo program was developed to simulate real data events as closely as possible. This program was used for many different tasks, including designing the experiment, testing the reconstruction program, studying data selection criteria, and studying experimental backgrounds. It was not directly used in the analysis of the data.

In the Monte Carlo program, the parent particle was generated in the downstream target using a Gaussian distribution in $x$ and $y$. The parent particle momentum was produced with a spectrum that matched the momentum spectrum of the real data sample.

The parent particles were then traced through the charged collimator and any that exited the collimator were allowed to randomly decay with their known lifetimes[40]. All subsequent unstable daughter particles were also allowed to decay randomly according to their known lifetimes. The daughter particles were then tracked through the spectrometer and bent in the momentum analyzing magnets.

The $x$ and $y$ position of each charged particle was calculated at each MWPC and SSD signal plane. Using this information and the chamber centers found from alignment studies, wire hits were generated for each charged track. The events were then required to pass the experimental trigger conditions. The hit wire information for events that passed the trigger conditions was written to files in the same format as the first level summary tapes. In addition, other information about the events, including generated kinematic parameters and information about the hit assignments were written to other files for use in checking the efficiency of the reconstruction and analysis programs.

In addition to generating "perfect" Monte Carlo events, it was possible to generate more realistic Monte Carlo events to make more detailed studies of the experimental acceptances. To do this, several other features were added to the program. Coulomb multiple scattering of the particles on the material in the spectrometer was one of the added features. The position and corresponding radiation lengths of the material in the spectrometer was compiled, and a random number generator was used to determine if a particle was scattered by some part of the apparatus. When scattering occurred, 
the direction of the particle was adjusted and tracking of the particle through the spectrometer continued. Studies of real data also revealed that a particle passing through a plane of a detector could cause two adjacent wires to register hits if the track passed through the central region between the two wires. The frequency of such occurrences was studied with real data and simulated in the Monte Carlo. The MWPC and SSD efficiencies discussed in Chapter 2 were also incorporated into the Monte Carlo program. Also, the existence of fringe fields in the Hyperon magnet and the analysis magnets, and their effect on the direction of charged particle tracks were studied and simulated.

It should be noted that the purpose of the Monte Carlo program was not to model the data in every possible detail. Rather it was intended to be a useful tool in understanding the experiment and the various reconstruction programs.

\subsection{Monte Carlo Program Performance}

To ensure that the Monte-Carlo-generated events accurately mocked the real data, comparisons were made between reconstructed Monte Carlo events and reconstructed real data events. Figures $3.2-3.5$ show comparisons of $m_{\Xi^{-}}, X_{T}, Y_{T}$, the $\Xi^{-}$and $\Lambda$ vertices, the $\Xi^{-}$and $\Lambda$ momentum spectra, $\chi^{2}{ }_{G} / D F$, and $\chi^{2}{ }_{\Lambda}$. These plots demonstrate that the Monte-Carlo-generated events adequately matched the real data events.

\subsection{Performance of the Reconstruction Process}

One reason that two retry processes were developed to improve the reconstruction can be see in Figure 3.6. This shows the difference between the Monte-Carlo-generated value and the reconstructed value of the center of mass decay angles on an event by event basis. In making this plot, only the first reconstruction process was used. These plots show that while this process generally does a good job of reproducing the generated
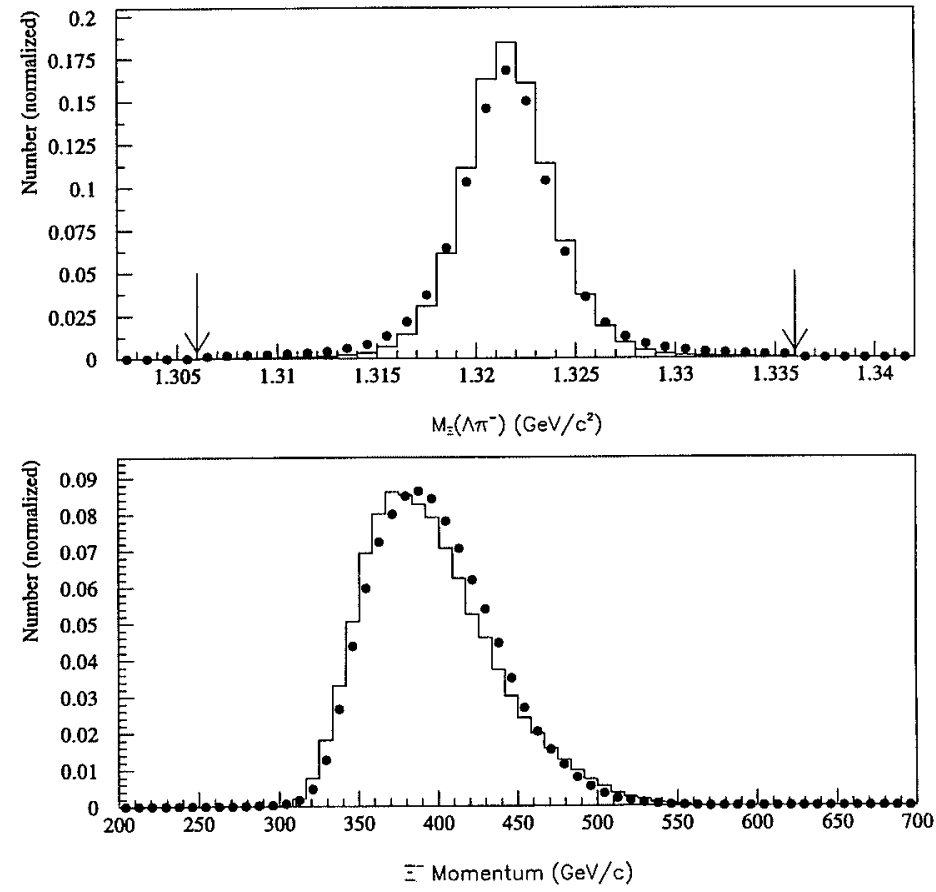

Figure 3.2: Comparison of $\Xi^{-}$mass and momentum for Monte Carlo (solid line) and real data (circles). The arrows indicate the data selection criteria. 

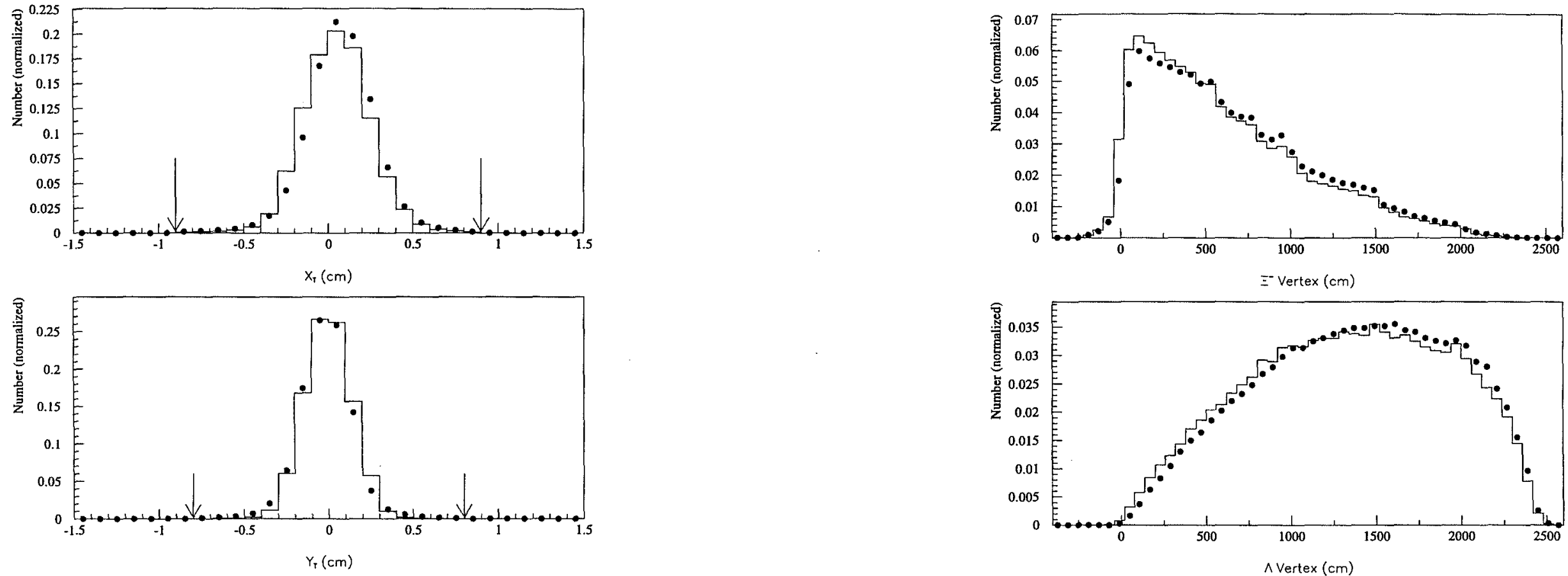

Figure 3.3: Comparison of $\mathrm{x}$ and $\mathrm{y}$ distributions at the downstream target for $\Xi^{-}$ events for Monte Carlo (solid line)and real data (circles). The arrows indicate the data selection criteria.

Figure 3.4: Comparison of $\Xi^{-}$and daughter $\Lambda$ vertex distributions for Monte Carlo (solid line) and real data (circles). 

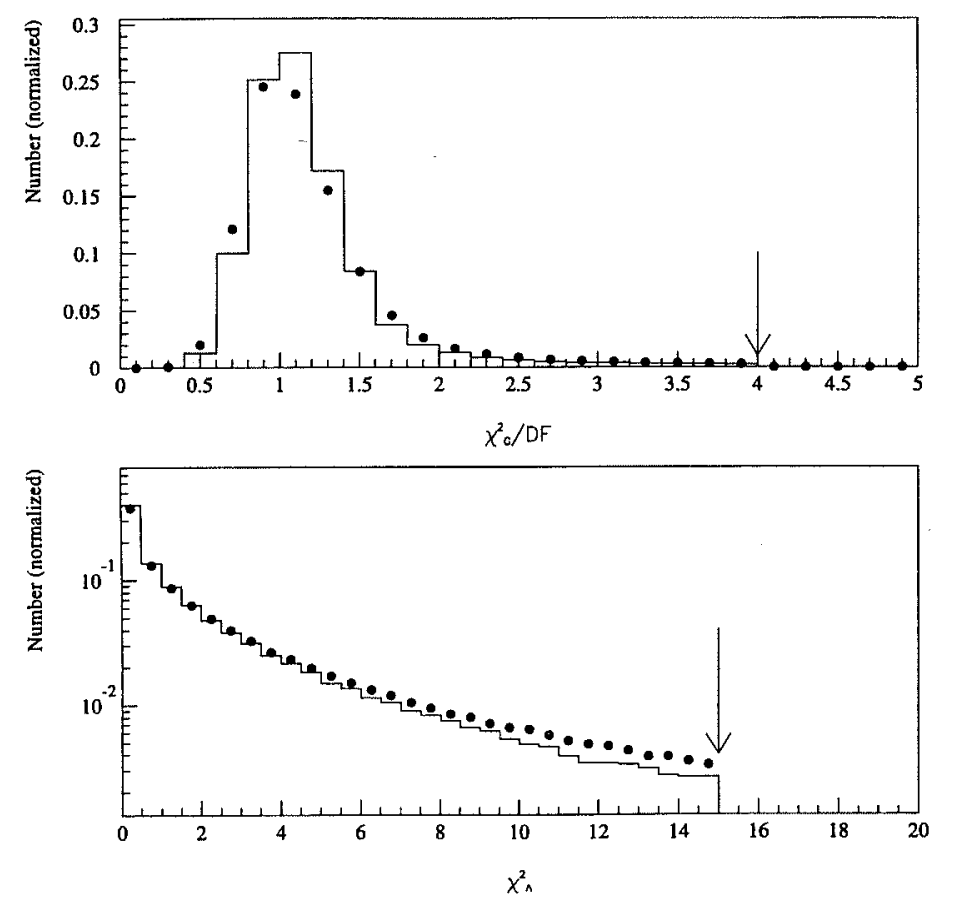

Figure 3.5: Comparison of $\chi_{G}^{2} / D F$ and $\chi_{\Lambda}^{2}$ distributions for Monte Carlo (solid line) and real data (circles) $\Xi^{-}$events.

cosine value, approximately $10 \%$ of the events are badly reconstructed. The main reason for this mismatch in the cosine distributions is problems with hit selection in the first reconstruction process. A comparison to the original Monte Carlo event information shows that only about $89 \%$ of the accepted events had the correct track assignment. In the polarization analysis, these mis-reconstructed events could have a substantial effect on the measured polarization.

It was found that the data selection criteria involving $\chi^{2}{ }_{G} / D F, \chi^{2}{ }_{\Lambda}, X_{T}, Y_{T}$, and the parent mass, listed in Table 3.4, increased the percentage of events with correct hit assignment to about $98 \%$. These new selection criteria reduced the number of accepted Monte Carlo events from $60 \%$ to $37 \%$.

The idea behind the two additional retry passes was to increase the percentage of events accepted by the reconstruction, especially events with small opening angles, without increasing the number of events with misassigned hits. The results of this effort are shown in Figure 3.7. This plot shows that now almost $100 \%$ of the events have their cosine values properly reconstructed. In addition, $51 \%$ of the Monte Carlo events that satisfied the criteria in Table 3.1 were accepted, and the percentage of events that had hit assignment problems was not increased.

To further study the effectiveness of the overall reconstruction process, extensive studies were made to ensure that the reconstruction adequately reproduced the MonteCarlo-generated properties of the events. Comparisons were made between the Monte Carlo generated and reconstructed values of the $\Xi^{-}$and $\Lambda$ vertex positions along the $\approx$ axis, and the $\Xi^{-}$and $\Lambda$ momenta. Similar comparisons were made using Monte Carlo generated $\Omega^{-}$events. It was found that the reconstruction process was able to adequately reproduce the Monte Carlo generated quantities. 

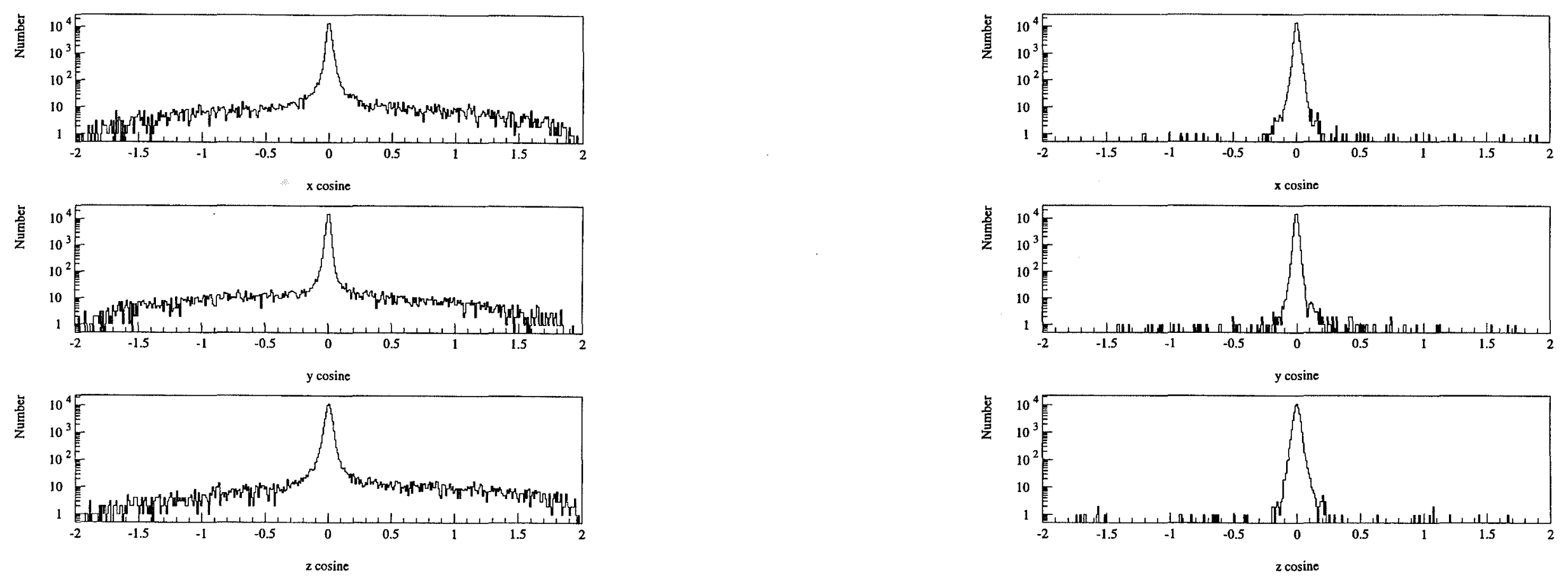

Figure 3.6: Difference between generated and reconstructed cosine values using only the first reconstruction process.

Figure 3.7: Difference between generated and reconstructed cosine values using all parts of the reconstruction process. 

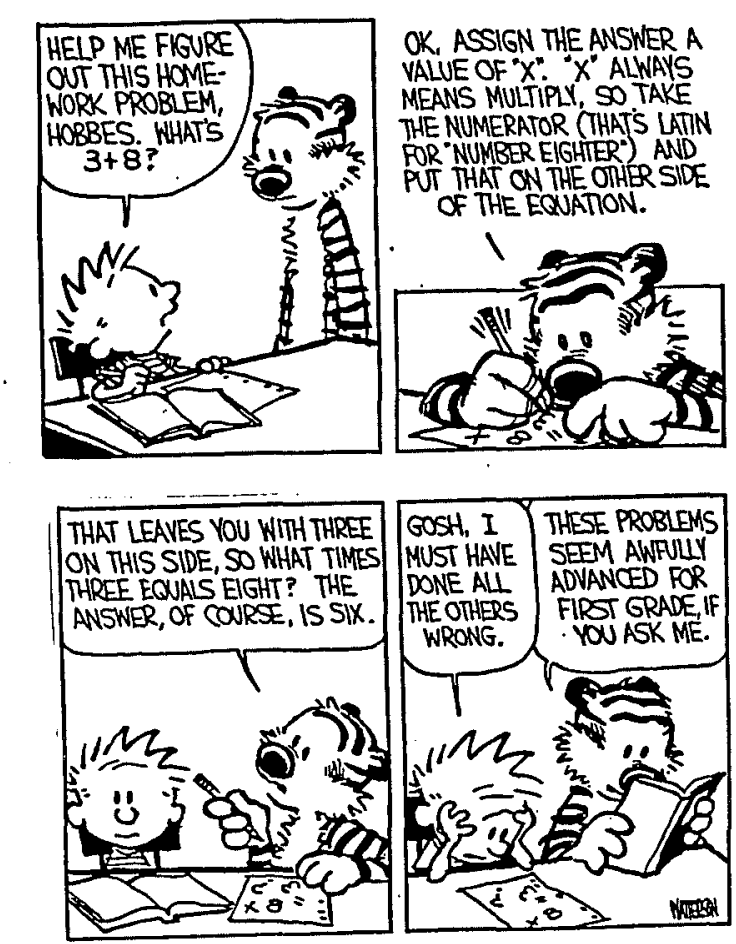

CALVIN AND HOBBES copyright Watterson. Dist. by UNIVERSAL PRESS SYNDICATE. Reprinted with permission. All rights reserved.

\section{Chapter 4}

\section{Polarization Analysis}

\section{$4.1 \quad \Xi^{-}$Polarization}

The decays $\Xi^{-} \rightarrow \Lambda+\pi^{-}$and $\Lambda \rightarrow p+\pi^{-}$both involve the decay of a spin $\frac{1}{2}$ particle into a spin $\frac{1}{2}$ particle and a spin 0 particle. As a result, the distributions of the daughter baryon in the rest frame of the parent, $\frac{d n}{d \Omega}$, obeys the following equation:

$$
\frac{d n}{d \Omega}=\frac{1}{4 \pi}(1+\alpha \vec{P} \cdot \hat{p})
$$

Here, $\vec{P}$ is the parent polarization, $\hat{p}$ is the direction of the daughter spin $\frac{1}{2}$ particle in the rest frame of the parent, and $\alpha$ is the parity violating helicity of the daughter spin $\frac{1}{2}$ particle[41]. This equation shows that unpolarized particles decay isotropically. Expressing Equation 4.1 in spherical coordinates and integrating over the azimuthal angle, $\phi$, yields

$$
\frac{d n}{d\left(\cos \theta_{i}\right)}=\frac{1}{2}\left(1+\alpha P_{i} \cos \theta_{i}\right)
$$

where $P_{i}$ is the component of the polarization along axis $\hat{i}$, and $\theta_{i}$ is the angle between the daughter particle direction and axis $\hat{i}$. The integration over $\phi$ that led to Equation 4.2 is only experimentally useful if there is little or no dependence of the daughter distribution on $\phi$ due to apparatus acceptance. 
Since the acceptance of the $\Lambda$ decay products was well understood, the measurement of the $\Xi^{-}$polarization was done by directly measuring the $\Lambda$ polarization. $\vec{P}_{\Lambda}$ and $\vec{P}_{\Xi}$ are related by the equation[42]:

$$
\vec{P}_{\Lambda}=\frac{\left(\alpha \Xi+\hat{\Lambda} \cdot \vec{P}_{\Xi}\right) \hat{\Lambda}+\beta_{\Xi}\left(\vec{P}_{\Xi} \times \hat{\Lambda}\right)+\gamma_{\Xi}\left(\hat{\Lambda} \times \vec{P}_{\Xi}\right) \times \hat{\Lambda}}{1+\alpha_{\Xi} \hat{\Lambda} \cdot \vec{P}_{\Xi}}
$$

Here, $\hat{\Lambda}$ is the direction of the $\Lambda$ in the $\Xi^{-}$rest frame and $\vec{P}_{\Xi}$ is the $\Xi^{-}$polarization. $\alpha \Xi, \beta \Xi$, and $\gamma \Xi$ are the weak decay parameters for $\Xi^{-} \rightarrow \Lambda+\pi^{-}$and obey the relation

$$
\alpha \Xi^{2}+\beta \Xi^{2}+\gamma \Xi^{2}=1
$$

It should be noted that a vector polarization is really only well defined in the rest frame of the polarized particle. Therefore, the quantities on the right hand side of Equation 4.3 are measured in the $\Xi^{-}$rest frame, and $\vec{P}_{\Lambda}$ is defined in the $\Lambda$ rest frame. This equation can be simplified by assuming time reversal invariance which implies $\beta_{\Xi}$ $=0[43]$. After making this substitution and rearranging terms, Equation 4.3 becomes:

$$
\vec{P}_{\Lambda}=\frac{\alpha \Xi \hat{\Lambda}+\left(\left(1-\gamma_{\Xi}\right) \hat{\Lambda} \cdot \vec{P}_{\Xi}\right) \hat{\Lambda}+\gamma_{\Xi} \vec{P}_{\Xi}}{1+\alpha \Xi \hat{\Lambda} \cdot \vec{P}_{\Xi}}
$$

This equation shows that a sample of unpolarized $\Xi^{-} \mathrm{s}$ can yield a sample of polarized $\Lambda s$ if the axes are selected in such a manner that the average value of $\hat{\Lambda}$ is large. If the average value of $\hat{\Lambda}$ is small, this term could be neglected.

Since the polarization was measured with respect to the spectrometer axes, and since $\hat{\Lambda}$ should be a random direction with respect to the spectrometer axes, terms involving $\hat{\Lambda} \cdot \vec{P}_{\Xi}$ should be small. Neglecting these terms in Equation 4.5 leads to a much simpler expression:

$$
\vec{P}_{\Lambda}=\alpha \Xi \hat{\Lambda}+\gamma_{\Xi} \vec{P}_{\Xi}
$$

\section{$4.2 \Omega^{-}$Polarization}

A similar expression can be derived to relate the polarization of the $\Omega^{-}$to the polarization of its daughter $\Lambda$. This derivation is complicated by the fact that the $\Omega^{-}$is not a spin $\frac{1}{2}$ particle. The spin of the $\Omega^{-}$has not actually been measured, but a spin of $\frac{3}{2}$ is predicted by the standard quark model and a spin of $\frac{1}{2}$ has been ruled out by experimental evidence[44, 45]. In this analysis, we assume a value of $\frac{3}{2}$. The relationship between $\vec{P}_{\Omega}$ and $\vec{P}_{\Lambda}$ is $[25,46,47]$

$$
\vec{P}_{\Lambda}=\frac{1}{5}\left(1+4 \gamma_{\Omega}\right) \vec{P}_{\Omega}
$$

Since $\alpha_{\Omega}$ is small [40] and time reversal invariance implies $\beta_{\Omega}=0$, Equation 4.4 leads to the conclusion that $\left|\gamma_{\Omega}\right| \approx 1$. With this substitution, Equation 4.7 reduces to

$$
\vec{P}_{\Lambda}= \begin{cases}\vec{P}_{\Omega} & \text { for } \gamma_{\Omega}=1 \\ -0.6 \vec{P}_{\Omega} & \text { for } \gamma_{\Omega}=-1\end{cases}
$$

A value of $\gamma_{\Omega}=+1$ is predicted by theory $[48,49,50]$ and favored by the data from this experiment [47].

\section{3 $\Lambda$ Polarization Measurement}

The $\Lambda$ polarization was measured by observing the distribution of the daughter protons from the decay $\Lambda \rightarrow p+\pi^{-}$. For this decay, Equation 4.2 becomes:

$$
\frac{d n_{p}}{d\left(\cos \theta_{i}\right)}=\frac{1}{2}\left(1+\alpha_{\Lambda} P_{\Lambda i} \cos \theta_{i}\right)
$$

This can be combined with Equation 4.6 to get

$$
\frac{d n_{p}}{d\left(\cos \theta_{i}\right)}=\frac{1}{2}\left[1+\left(\alpha_{\Lambda} \alpha_{\Xi} \Lambda_{i}+\alpha_{\Lambda} \gamma_{\Xi} P_{\Xi i}\right) \cos \theta_{i}\right]
$$

Here, $P_{\Xi i}$ and $\Lambda_{i}$ are the components of $\vec{P}_{\Xi}$ and $\hat{\Lambda}$ along axis $\hat{i}$.

Combining Equation 4.8 with Equation 4.9, leads to a similar expression for the $\Omega^{-}$ case.

$$
\frac{d n_{p}}{d\left(\cos \theta_{i}\right)}=\frac{1}{2}\left[1+\alpha_{\Lambda} P_{\Omega i} \cos \theta_{i}\right]
$$


If the experimental apparatus and reconstruction programs both had perfect acceptances, $\vec{P}_{\Xi}$ and $\vec{P}_{\Omega}$ could be found by fitting the proton distribution to Equations 4.10 and 4.11 and finding the values of the polarizations that gave the best fit. However, this is not the case and a method of correcting for the finite acceptance of the spectrometer and reconstruction must be used.

A hybrid Monte Carlo technique[51] was used to map out the experimental acceptance in $\cos \theta_{i}$ using fake events. These fake events were kinematically identical to a given real event in every way except that the value of $\cos \theta_{i}$ was randomly chosen between -1 and +1 . They were required to pass through the same data selection criteria used on real data events. These criteria included known chamber apertures and topology requirements. The topology criteria are discussed in further detail in Appendix B. In addition to these selection criteria, a map of wires in the MWPCs that were very noisy or never registered hits was compiled and any fake event hit that fell on one of these wires was removed. For every real event, fake events were generated until 10 such events passed the selection criteria. Statistical studies indicated that the requirement of 10 accepted hybrid events would be large enough to allow the sampling uncertainties due to the hybrid Monte Carlo to be neglected in comparison to the statistical uncertainties of the real data.

When $P_{\Lambda i}=0$, the fake distribution was expected to agree with the real $\cos \theta_{i}$ distribution since both sets of events would have passed through the same data selection criteria. When $P_{\Lambda i} \neq 0$, however, the distributions will not match due to the asymmetric $\cos \theta_{i}$ distribution of the real data events. To correct this, Equation 4.10 was used to generate a weighting factor, $W_{j k}$, for each fake event.

$$
W_{j k}=\frac{1+\left(\alpha_{\Lambda} \alpha_{\Xi} \hat{\Lambda}_{i}+\alpha_{\Lambda} \gamma_{\Xi} P_{\Xi i}\right) \cos \theta_{i j k}}{1+\left(\alpha_{\Lambda} \alpha_{\Xi} \hat{\Lambda}_{i}+\alpha_{\Lambda} \gamma \Xi P_{\Xi i}\right) \cos \theta_{i} j}
$$

where subscript $j$ refers to the real event, and subscript $j k$ refers to the $k$ th fake event generated for real event $j$. A similar expression for the $P_{\Omega}$ analysis was derived from
Equation 4.11.

$$
W_{j k}=\frac{1+\alpha_{\Lambda} P_{\Omega i} \cos \theta_{i j k}}{1+\alpha_{\Lambda} P_{\Omega i} \cos \theta_{i j}}
$$

The $\cos \theta_{i}$ distributions were divided into twenty bins, and a bin by bin comparison of the real and fake $\cos \theta_{i}$ distributions was made. A $\chi^{2}$ was calculated for each bin using the following equation

$$
\chi^{2}=\frac{\left(n_{\tau}-n_{f} N_{r} / N_{f}\right)^{2}}{n_{\tau}}
$$

where $n_{r}$ and $n_{f}$ are the contents of a given $\cos \theta_{i}$ bin for the real and fake distributions respectively. $N_{r}$ and $N_{f}$ are the total number of events in the real and fake distributions. For a given bin, $n_{f}$ is given by

$$
n_{f}=\sum_{j k} W_{j k}
$$

The overall $\chi^{2}$ is calculated by summing the $\chi^{2}$ s from the individual bins. The polarization, $P_{\Xi i}$ is then varied to find the minimum $\chi^{2}$. Equation 4.12 is expanded as a power series in $\alpha_{\Lambda} \gamma_{\Xi} P_{\Xi i}$ and substituted into Equation 4.15 so that

$$
n_{f}=\sum_{j k} W_{j k}=\left[\sum_{j k} A_{j k}\right]+\left[\sum_{j k} B_{j k}\right]\left(\alpha_{\Lambda} \gamma_{\Xi} P_{\Xi i}\right)-\left[\sum_{j k} C_{j k}\right]\left(\alpha_{\Lambda} \gamma \Xi P_{\Xi i}\right)^{2}+\cdots
$$

where

$$
\begin{gathered}
A_{j k}=\frac{1+\alpha_{\Lambda} \alpha_{\Xi} \hat{\Lambda}_{i} \cos \theta_{j k}}{1+\alpha_{\Lambda} \alpha_{\Xi} \hat{\Lambda}_{i} \cos \theta_{j}} \\
B_{j k}=\frac{\cos \theta_{j k}-\cos \theta_{j}}{\left(1+\alpha_{\Lambda} \alpha_{\Xi} \hat{\Lambda}_{i} \cos \theta_{j}\right)^{2}} \\
C_{j k}=\frac{\cos \theta_{j}\left(\cos \theta_{j k}-\cos \theta_{j}\right)}{\left(1+\alpha_{\Lambda} \alpha_{\Xi} \hat{\Lambda}_{i} \cos \theta_{j}\right)^{3}}
\end{gathered}
$$

The sums in Equation 4.16 are not dependent on $P_{\Xi i}$ so a single pass can be made through the events to evaluate the sums, and then $P_{\Xi i}$ can be varied to minimize the overall $\chi^{2}$. The uncertainty in the measurement of $P_{\Xi i}$ is evaluated by varying $P_{\Xi i}$ on either side of the best value until the overall $\chi^{2}$ changes by 1 from the minimum value. 
Once a value has been found for $P_{\Xi i}$, a second pass through the hybrid Monte Carlo was then made, using this value of $\vec{P}_{\Xi}$ to consider the $\hat{\Lambda} \cdot \vec{P}_{\Xi}$ terms in Equation 4.5 that were neglected in the first pass. New weights, $W_{j k}$, were calculated and the power series expansion was repeated to find a new value of $P_{\Xi i}$. It was found that no more than one iteration was needed for the value of $\vec{P}_{\Xi}$ to converge. The measurement of $P_{\Omega}$ was performed using the same technique.

This analysis method was tested using Monte Carlo data that was generated to simulate a polarized data sample. It was found that the analysis process adequately reproduced that input polarization. This will be discussed further in Chapter 5 .

\subsection{The Bias Cancellation}

The method described above only accounts for the geometric acceptance of the spectrometer and for the data selection criteria imposed on the real data sample. It does not account for acceptances due to other reconstruction acceptances or effects due to multiple scattering or any unidentified processes. The value of the asymmetry, $\alpha_{\Lambda} \gamma \Xi P_{\Xi i}$ or $\alpha_{\Lambda} P_{\Omega i}$, measured by this technique is actually a sum of two components. These components are a term due to the real polarization signal and a "bias" term which is due to uncorrected imperfections in the experimental apparatus or the reconstruction program. Any of these factors which has a non-uniform effect on the $\cos \theta_{i}$ distribution will also increase the overall $\chi^{2}$ in addition to introducing a bias.

Since the sign of the polarization is defined relative to the production plane, the sign of the polarization is reversed when the production angle is reversed. However, since the biases depend only on the nature of the spectrometer and reconstruction programs, they should not be affected by a change in the production angle. This can be exploited to separate the real polarization from the biases. The measured asymmetries, $A_{+}$and $A_{-}$for the positive and negative production angle, can be written in terms of the bias
B, and the real polarization signal $P$ as

$$
\begin{aligned}
& A_{+}=B+P \\
& A_{-}=B-P
\end{aligned}
$$

The bias and real signal components are then extracted from the measured asymmetries:

$$
\begin{aligned}
& P=\frac{A_{+}-A_{-}}{2} \\
& B=\frac{A_{+}+A_{-}}{2}
\end{aligned}
$$

where $P=\alpha_{\Lambda} \gamma_{\Xi} P \Xi$ or $P=\alpha_{\Lambda} P_{\Omega}$.

This technique could be used to extract the polarization directly from the reconstructed data sample, without using the hybrid Monte Carlo procedure. However, any biases which do depend on the production angle would be incorporated into the measured asymmetry. In this experiment, there were possible production angle dependent biases that we wished to consider, so the hybrid Monte Carlo technique was used. The major possible source of production angle dependent biases was the spectrometer, since the beam illuminates different parts of the spectrometer at the different production angles.

\subsection{Calculation of Target Polarization}

Due to the parity conservation argument described in Chapter 1 , the only allowed polarization direction is perpendicular to the production plane, which in this experiment is the $x$ direction. The field in the Hyperon magnet precessed this polarization vector into the $x-z$-plane. The polarization at the production target could be extracted by fitting the measured $P_{x}$ and $P_{z}$ values to the angle, $\Phi$, through which the parent particle precesses in the hyperon magnet. The precession angle is defined in terms of 
known quantities using the expression:

$$
\Phi=\left[\frac{\mu}{c}-\frac{q}{m c^{2}}\right] \int B d l
$$

where $\mu$ is the known magnetic moment, $q$ is the charge of the hyperon, $m$ is the mass of the hyperon, and $\int B d l$ is the known field integral in the sweeper magnet. For the data taken with a current of -750 Amps in the hyperon magnet, the $\int B d l$ was -17.48 T-m, and for the data with a hyperon magnet current of $-2900 \mathrm{Amps}$, the $\int B d l$ was $-24.36 \mathrm{~T}-\mathrm{m}$. In the $\Xi^{-}$analysis, the current world average magnetic moment value of $-0.6507 \pm 0.0025$ was used [40]. The magnetic moment used in the $\Omega^{-}$analysis was the value of $-2.024 \pm 0.056$ measured by this experiment [52].

For each data sample, the polarization at the target, $P_{t g t}$, is calculated by varying $P_{t g t}$ to minimize the $\chi^{2}$ function:

$$
\chi^{2}=\sum_{i}\left[\frac{\left(P_{x i}-P_{t g t} \cos \Phi\right)^{2}}{\sigma_{x i}^{2}}+\frac{\left(P_{z i}-P_{t g t} \sin \Phi\right)^{2}}{\sigma_{z i}^{2}}\right]
$$

The subscript $i$ runs over the momentum bins, $P_{x i}$ and $P_{z i}$ are the measured polarization components, and $\sigma_{x i}^{2}$ and $\sigma_{z i}^{2}$ are the uncertainties of the measured polarization components.

The same expression is used in making a magnetic moment measurement, however, in this case the magnetic moment is also treated as a variable parameter.

In this experiment, in the analysis of the $\Xi^{-}$data, the value of $\mu_{\Xi-}$ was treated as fixed. For the $\Omega^{-}$data, the magnetic moment was allowed to vary, so that a measurement of $\mu_{\Omega}$ - could be made [52].
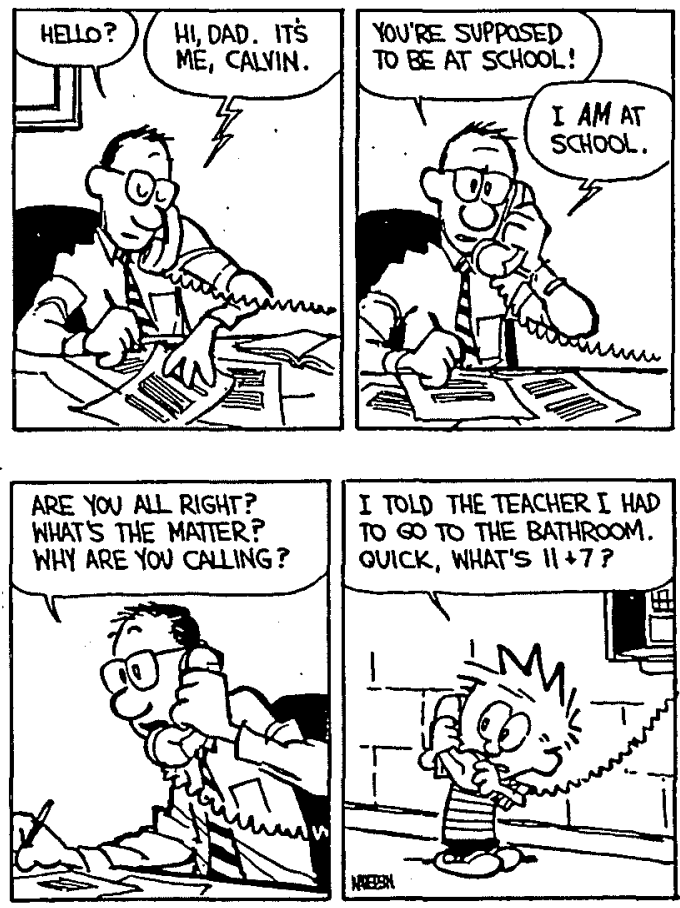

CALVIN AND HOBBES copyright Watterson. Dist. by UNIVERSAL PRESS SYNDICATE. Reprinted with permission. All rights reserved. 


\section{Chapter 5}

\section{Results and Systematic}

\section{Uncertainty Studies}

\subsection{Polarization Results}

All of the unpolarized neutral beam production and polarized neutral beam production data were reconstructed and analyzed for polarization using the methods described in the preceeding chapters.

\subsubsection{Unpolarized Neutral Beam Production Data}

Table 5.1 gives the measured asymmetries $\left(\alpha_{\Lambda} \gamma_{\Xi} P \Xi\right)$ and biases as a function of momentum for the $\Xi^{-}$unpolarized neutral beam production data for a current of -2900 amps in the hyperon magnet. Table 5.2 gives the measured asymmetries $\left(\alpha_{\Lambda} P_{\Omega}\right)$ and biases for the $\Omega^{-}$data for this production mode. Figures 5.1 and 5.2 show the unpolarized neutral beam production asymmetries and biases plotted as a function of momentum for both the $\Xi^{-}$and $\Omega^{-}$samples. The momentum averaged asymmetries and biases are given in Table 5.5 for the $\Xi^{-}$and Table 5.6 for the $\Omega^{-}$.

\begin{tabular}{|c|c|c|c|c|}
\hline $\begin{array}{c}\text { Mom. } \\
(\mathrm{GeV} / \mathrm{c})\end{array}$ & $\begin{array}{c}\text { Number of } \\
\text { Events (1000s) }\end{array}$ & View & $\begin{array}{c}\text { Asymmetry } \\
\alpha_{\Lambda} \gamma_{\Xi} P \Xi\end{array}$ & Bias \\
\hline \multirow{3}{*}{346.} & \multirow{3}{*}{1393} & $\mathrm{x}$ & $-0.0029 \pm 0.0016$ & $0.0247 \pm 0.0016$ \\
\hline & & $\mathrm{y}$ & $0.0000 \pm 0.0017$ & $0.0346 \pm 0.0017$ \\
\hline & & $\mathrm{z}$ & $-0.0038 \pm 0.0020$ & $-0.0658 \pm 0.0020$ \\
\hline \multirow{3}{*}{369.} & \multirow{3}{*}{1655} & $\mathrm{x}$ & $0.0012 \pm 0.0014$ & $-0.0082 \pm 0.0014$ \\
\hline & & $\mathrm{y}$ & $0.0029 \pm 0.0015$ & $0.0224 \pm 0.0015$ \\
\hline & & $z$ & $-0.0096 \pm 0.0017$ & $-0.0197 \pm 0.0017$ \\
\hline \multirow{3}{*}{383.} & \multirow{3}{*}{1793} & $\mathrm{x}$ & $0.0007 \pm 0.0013$ & $-0.0029 \pm 0.0013$ \\
\hline & & $\mathrm{y}$ & $0.0023 \pm 0.0014$ & $0.0174 \pm 0.0014$ \\
\hline & & $\mathrm{z}$ & $-0.0061 \pm 0.0016$ & $0.0000 \pm 0.0016$ \\
\hline \multirow{3}{*}{399.} & \multirow{3}{*}{1684} & $\mathrm{x}$ & $0.0056 \pm 0.0013$ & $-0.0042 \pm 0.0013$ \\
\hline & & $\mathrm{y}$ & $0.0041 \pm 0.0015$ & $0.0153 \pm 0.0015$ \\
\hline & & $\mathrm{z}$ & $-0.0054 \pm 0.0017$ & $0.0096 \pm 0.0017$ \\
\hline \multirow{3}{*}{418.} & \multirow{3}{*}{1814} & $\mathrm{x}$ & $0.0033 \pm 0.0013$ & $-0.0044 \pm 0.0013$ \\
\hline & & $y$ & $0.0023 \pm 0.0014$ & $0.0128 \pm 0.0014$ \\
\hline & & $z$ & $0.0023 \pm 0.0016$ & $0.0095 \pm 0.0016$ \\
\hline \multirow{3}{*}{453.} & \multirow{3}{*}{1633} & $\mathrm{x}$ & $0.0005 \pm 0.0015$ & $-0.0100 \pm 0.0015$ \\
\hline & & $\mathrm{y}$ & $-0.0003 \pm 0.0015$ & $0.0188 \pm 0.0015$ \\
\hline & & $\mathrm{z}$ & $0.0005 \pm 0.0018$ & $0.0264 \pm 0.0018$ \\
\hline
\end{tabular}

Table 5.1: Measured $\Xi^{-}$asymmetries and biases as a function of momentum for the unpolarized neutral beam production sample with the sweeper magnet set at -2900 amps.

\begin{tabular}{c|c|c|c|c}
$\begin{array}{c}\text { Mom. } \\
(\mathrm{GeV} / \mathrm{c})\end{array}$ & $\begin{array}{c}\text { Number of } \\
\text { Events (1000s) }\end{array}$ & View & $\begin{array}{c}\text { Asymmetry } \\
\alpha_{\Lambda} P_{\Omega}\end{array}$ & Bias \\
\hline 348. & 42 & $\mathrm{x}$ & $0.0215 \pm 0.0092$ & $-0.0092 \pm 0.0092$ \\
& & $\mathrm{y}$ & $-0.0005 \pm 0.0097$ & $0.0151 \pm 0.0097$ \\
& & $\mathrm{z}$ & $0.0255 \pm 0.0115$ & $0.0208 \pm 0.0115$ \\
\hline & & $\mathrm{x}$ & $0.0048 \pm 0.0084$ & $0.0086 \pm 0.0084$ \\
380. & 42 & $\mathrm{y}$ & $0.0125 \pm 0.0094$ & $0.0363 \pm 0.0094$ \\
& & $\mathrm{z}$ & $0.0204 \pm 0.0101$ & $0.0394 \pm 0.0101$ \\
\hline & & $\mathrm{x}$ & $0.0207 \pm 0.0090$ & $-0.0006 \pm 0.0090$ \\
403. & 37 & $\mathrm{y}$ & $0.0117 \pm 0.0099$ & $-0.0042 \pm 0.0099$ \\
& & $\mathrm{z}$ & $0.0311 \pm 0.0103$ & $0.0524 \pm 0.0311$ \\
\hline & & $\mathrm{x}$ & $0.0272 \pm 0.0086$ & $0.0150 \pm 0.0086$ \\
443. & 44 & $\mathrm{y}$ & $0.0065 \pm 0.0090$ & $0.0088 \pm 0.0090$ \\
& & $\mathrm{z}$ & $0.0149 \pm 0.0100$ & $0.0766 \pm 0.0100$ \\
\hline
\end{tabular}

Table 5.2: Measured $\Omega^{-}$asymmetries and biases as a function of momentum for the unpolarized neutral beam production sample with the sweeper magnet set at -2900 amps. 

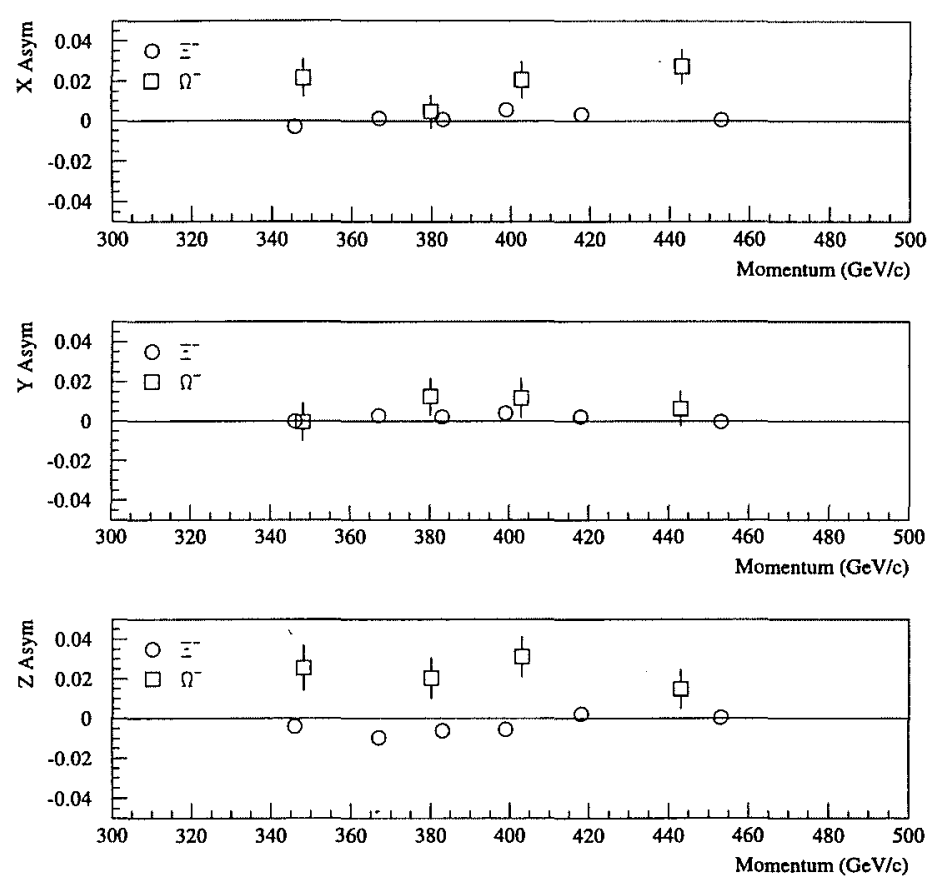

Figure 5.1: Asymmetries $\left(\alpha_{\Lambda} \gamma_{\Xi} P_{\Xi}\right.$ and $\left.\alpha_{\Lambda} P_{\Omega}\right)$ for the unpolarized neutral beam production sample with the sweeper magnet set at -2900 Amps.
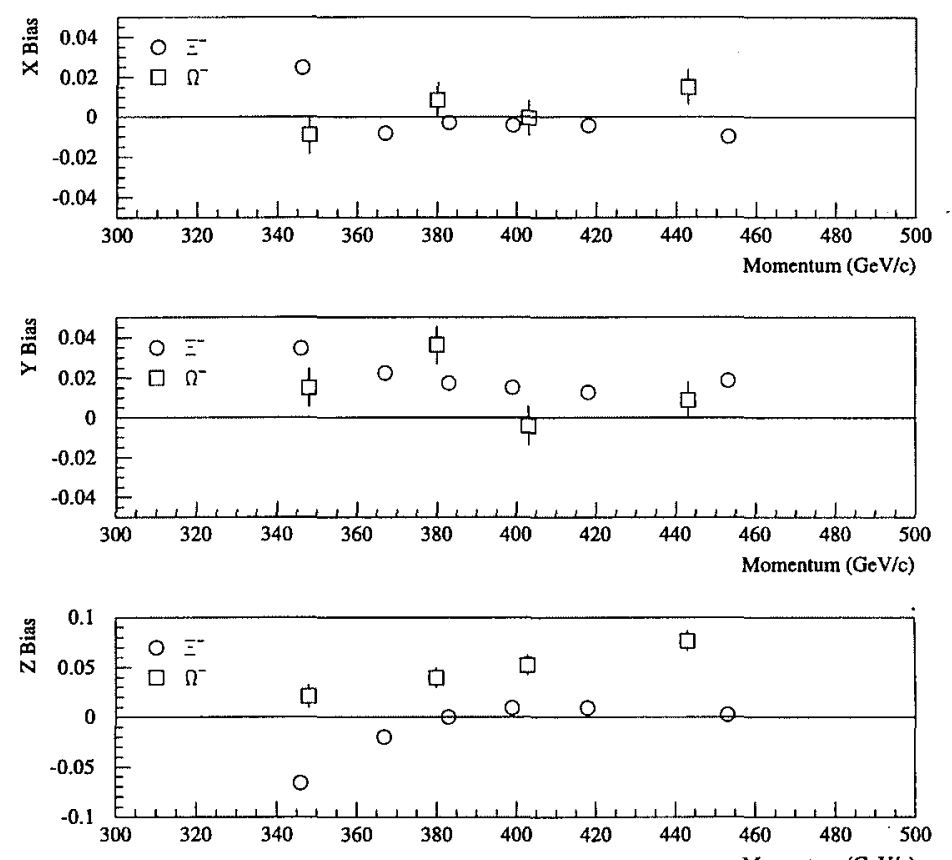

Figure 5.2: Biases for the unpolarized neutral beam production sample with the sweeper magnet set at -2900 Amps. 


\begin{tabular}{c|c|c|c|c}
$\begin{array}{c}\text { Mom. } \\
(\mathrm{GeV} / \mathrm{c})\end{array}$ & $\begin{array}{c}\text { Number of } \\
\text { Events }(1000 \mathrm{~s})\end{array}$ & View & $\begin{array}{c}\text { Asymmetry } \\
\alpha_{\Lambda} \gamma_{\Xi} P_{\Xi}\end{array}$ & Bias \\
\hline \multirow{2}{*}{259.} & 731 & $\mathrm{x}$ & $-0.0063 \pm 0.0022$ & $-0.0500 \pm 0.0022$ \\
& & $\mathrm{y}$ & $0.0021 \pm 0.0024$ & $0.0374 \pm 0.0024$ \\
$\mathrm{z}$ & $-0.0041 \pm 0.0031$ & $-0.0766 \pm 0.0031$ \\
\hline & & $\mathrm{x}$ & $-0.0032 \pm 0.0019$ & $-0.0053 \pm 0.0019$ \\
282. & 813 & $\mathrm{y}$ & $0.0066 \pm 0.0022$ & $0.0235 \pm 0.0022$ \\
& & $\mathrm{z}$ & $-0.0064 \pm 0.0025$ & $-0.0446 \pm 0.0064$ \\
\hline & & $\mathrm{x}$ & $-0.0070 \pm 0.0019$ & $-0.0002 \pm 0.0019$ \\
299. & \multirow{2}{*}{827} & $\mathrm{y}$ & $0.0032 \pm 0.0022$ & $0.0180 \pm 0.0022$ \\
& & $\mathrm{z}$ & $-0.0055 \pm 0.0023$ & $-0.0258 \pm 0.0023$ \\
\hline 318. & \multirow{2}{*}{745} & $\mathrm{x}$ & $-0.0050 \pm 0.0021$ & $-0.080 \pm 0.0021$ \\
& & $\mathrm{y}$ & $0.0023 \pm 0.0023$ & $0.0255 \pm 0.0023$ \\
& & $\mathrm{z}$ & $0.0000 \pm 0.0025$ & $-0.0049 \pm 0.0025$ \\
\hline \multirow{2}{*}{349.} & \multirow{2}{*}{645} & $\mathrm{x}$ & $-0.0084 \pm 0.0023$ & $-0.0294 \pm 0.0023$ \\
& & $\mathrm{y}$ & $0.0014 \pm 0.0025$ & $0.0303 \pm 0.0025$ \\
& & $\mathrm{z}$ & $0.0069 \pm 0.0028$ & $0.0269 \pm 0.0028$ \\
\hline
\end{tabular}

Table 5.3: Measured $\Xi^{-}$asymmetries and biases as a function of momentum for the unpolarized neutral beam production sample with the sweeper magnet set at $-750 \mathrm{amps}$.

Table 5.3 gives the measured asymmetries $\left(\alpha_{\Lambda} \gamma_{\Xi} P_{\Xi}\right)$ and biases as a function of momentum for the unpolarized neutral beam production data for a current of -750 amps in the hyperon magnet. Table 5.4 gives the same results for the $\Omega^{-} \mathrm{s}$ in this data sample. Figures 5.3 and 5.4 show the asymmetries and biases of this sample plotted as a function of momentum. The momentum averaged asymmetries and biases are given in Table 5.5 for the $\Xi^{-}$and Table 5.6 for the $\Omega^{-}$.

In this experiment, the only parity conserving direction for the polarization at the second target was $\hat{x}$. The hyperon magnet precessed this polarization vector into the $\hat{x}-\hat{z}$ plane. Therefore, any polarization would be indicated by measured non-zero asymmetries in the $\hat{x}$ and $\hat{z}$-directions. The precession angle depends only on the magnetic moment of the particle and the magnetic field through which the particle passes. The precession angle should be momentum independent. From Tables 5.1 and 5.3 and Figures 5.1 and 5.3 it can be seen that there is no clear polarization signal for the

\begin{tabular}{c|c|c|c|c}
$\begin{array}{c}\text { Mom. } \\
(\mathrm{GeV} / \mathrm{c})\end{array}$ & $\begin{array}{c}\text { Number of } \\
\text { Events (1000s) }\end{array}$ & View & $\begin{array}{c}\text { Asymmetry } \\
\alpha_{\Lambda} P_{\Omega}\end{array}$ & Bias \\
\hline \multirow{2}{*}{260.} & \multirow{2}{*}{10} & $\mathrm{x}$ & $0.0140 \pm 0.0202$ & $-0.0232 \pm 0.0202$ \\
& & $\mathrm{y}$ & $-0.0013 \pm 0.0216$ & $0.0446 \pm 0.0216$ \\
& $\mathrm{z}$ & $0.0166 \pm 0.0314$ & $-0.1401 \pm 0.0314$ \\
\hline \multirow{2}{*}{288.} & \multirow{2}{*}{15} & $\mathrm{x}$ & $0.0434 \pm 0.0144$ & $-0.0002 \pm 0.0144$ \\
& & $\mathrm{y}$ & $0.0203 \pm 0.0172$ & $0.0425 \pm 0.0172$ \\
& & $\mathrm{z}$ & $0.0102 \pm 0.0208$ & $-0.0104 \pm 0.0208$ \\
\hline 312. & \multirow{2}{*}{13} & $\mathrm{x}$ & $0.0235 \pm 0.0157$ & $-0.0144 \pm 0.0157$ \\
& & $\mathrm{y}$ & $-0.0094 \pm 0.0185$ & $-0.0066 \pm 0.0185$ \\
& & $\mathrm{z}$ & $0.0187 \pm 0.0199$ & $-0.0029 \pm 0.0199$ \\
\hline 349. & \multirow{2}{*}{13} & $\mathrm{x}$ & $-0.0163 \pm 0.0171$ & $0.0154 \pm 0.0171$ \\
& & $\mathrm{y}$ & $0.0163 \pm 0.0180$ & $0.0250 \pm 0.0180$ \\
& & $\mathrm{z}$ & $0.0117 \pm 0.0188$ & $0.0636 \pm 0.0188$ \\
\hline
\end{tabular}

Table 5.4: Measured $\Omega^{-}$asymmetries and biases as a function of momentum for the unpolarized neutral beam production sample with the sweeper magnet set at -750 amps.

\begin{tabular}{c|c|c|c|c|c}
$\begin{array}{c}\text { Sweeper } \\
\text { Current }\end{array}$ & $\begin{array}{c}\text { Mom. } \\
(\mathrm{GeV} / \mathrm{c})\end{array}$ & $\begin{array}{c}\text { Number of } \\
\text { Events }(1000 \mathrm{~s})\end{array}$ & View & $\begin{array}{c}\text { Asymmetry } \\
\alpha_{\Lambda} \gamma_{\Xi} P_{\Xi}\end{array}$ & Bias \\
\hline & & & $\mathrm{x}$ & $-0.0062 \pm 0.0009$ & $-0.0165 \pm 0.0009$ \\
750 & 300. & 3768 & $\mathrm{y}$ & $0.0032 \pm 0.0010$ & $0.0263 \pm 0.0010$ \\
Amps & & & $\mathrm{z}$ & $-0.0027 \pm 0.0012$ & $-0.0236 \pm 0.0012$ \\
\hline & & & $\mathrm{x}$ & $0.0019 \pm 0.0006$ & $-0.0018 \pm 0.0006$ \\
2900 & 395. & 9971 & $\mathrm{y}$ & $0.0019 \pm 0.0006$ & $0.0195 \pm 0.0006$ \\
Amps & & & $\mathrm{z}$ & $-0.0040 \pm 0.0007$ & $-0.0045 \pm 0.0007$ \\
\hline
\end{tabular}

Table 5.5: Overall unpolarized neutral beam production $\Xi^{-}$sample asymmetries and biases for the two sweeper magnet settings.

\begin{tabular}{c|c|c|c|c|c}
$\begin{array}{c}\text { Sweeper } \\
\text { Current }\end{array}$ & $\begin{array}{c}\text { Mom. } \\
(\mathrm{GeV} / \mathrm{c})\end{array}$ & $\begin{array}{c}\text { Number of } \\
\text { Events }(1000 \mathrm{~s})\end{array}$ & View & $\begin{array}{c}\text { Asymmetry } \\
\alpha_{\Lambda} P_{\Omega}\end{array}$ & Bias \\
\hline & & & $\mathrm{x}$ & $0.0186 \pm 0.0083$ & $-0.0032 \pm 0.0083$ \\
750 & 304. & 50 & $\mathrm{y}$ & $0.0072 \pm 0.0093$ & $0.0263 \pm 0.0093$ \\
Amps & & & $\mathrm{z}$ & $0.0140 \pm 0.0109$ & $-0.0010 \pm 0.0109$ \\
\hline & & & $\mathrm{x}$ & $0.0179 \pm 0.0044$ & $0.0044 \pm 0.0044$ \\
2900 & 394. & 167 & $\mathrm{y}$ & $0.0073 \pm 0.0048$ & $0.0141 \pm 0.0048$ \\
Amps & & & $\mathrm{z}$ & $0.0218 \pm 0.0053$ & $0.0496 \pm 0.0053$ \\
\hline
\end{tabular}

Table 5.6: Overall unpolarized neutral beam production $\Omega^{-}$sample asymmetries and biases for the two sweeper magnet settings. 

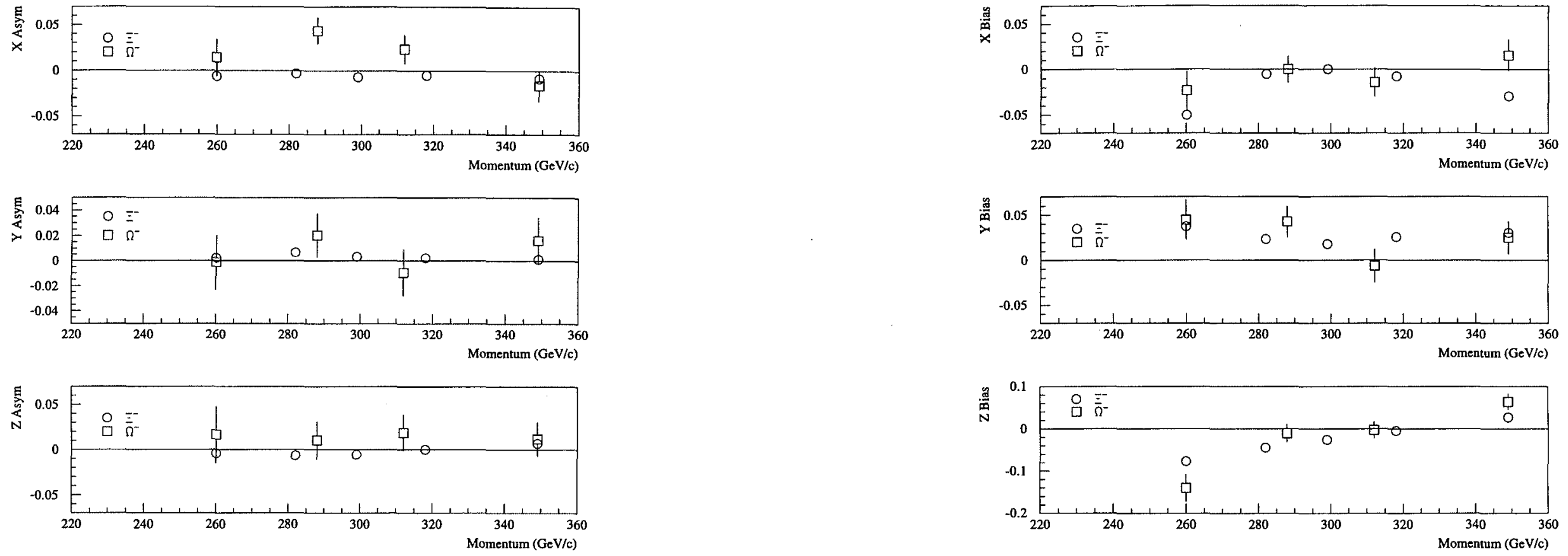

Figure 5.3: Asymmetries $\left(\alpha_{\Lambda} \gamma_{\xi} P_{\Xi}\right.$ and $\left.\alpha_{\Lambda} P_{\Omega}\right)$ for the unpolarized neutral beam production sample with the sweeper magnet set at -750 Amps.

Figure 5.4: Biases for the unpolarized neutral beam production sample with the sweeper magnet set at -750 Amps. 
$\Xi^{-}$data. Figure 5.5 shows this more clearly. This figure shows the $\Xi^{-}$and $\Omega^{-}$precession angle, $\Phi=\arctan \left(P_{z} / P_{x}\right)$, for the various momentum bins for the $-2900 \mathrm{amp}$ data. The expected precession angles, calculated using the known magnetic moments, are also shown. All the $\Omega^{-}$points are consistent with the expected precession angle, indicating a $\hat{x}$ polarization at the target being precessed into the $\hat{x}-\hat{x}^{-}$plane. The $\Xi^{-}$ sample does not show a similar consistency. This indicates that the $\Xi^{-}$sample was not polarized.

\subsubsection{Polarized Neutral Beam Production Data}

Table 5.7 gives the measured asymmetries $\left(\alpha_{\Lambda} \gamma_{\Xi} P_{\Xi}\right)$ and biases as a function of momentum for the polarized neutral beam $\Xi^{-}$data. Table 5.8 gives the measured asymmetries and biases for the $\Omega^{-}$s produced using the polarized neutral beam technique. Figures 5.6 and 5.7 show the measured asymmetries and biases plotted as a function of momentum for the $\Xi^{-}$and $\Omega^{-}$data. The momentum averaged asymmetries and biases are given in Table 5.9 for the $\Xi^{-}$and Table 5.10 for the $\Omega^{-}$.

Figure 5.8 shows the measured precession angles for the $\Xi^{-}$and $\Omega^{-}$events in this data sample. These figures show that measured precession angles agree with the expected value, indicating that both the $\Xi^{-}$and $\Omega^{-}$samples are polarized.

The polarization at the production target was extracted by fitting to a single magnetic moment using the method described in section 4.5. The target polarizations for the different samples are given in Table 5.11 and are plotted in Figure 5.9.

\subsubsection{Discussion of Biases}

Figure 5.10 shows the $\Xi^{-}$biases for the two unpolarized neutral beam data samples and the polarized neutral beam sample. This figure shows that biases for the polarized neutral beam sample and the unpolarized neutral beam sample with a current of -2900
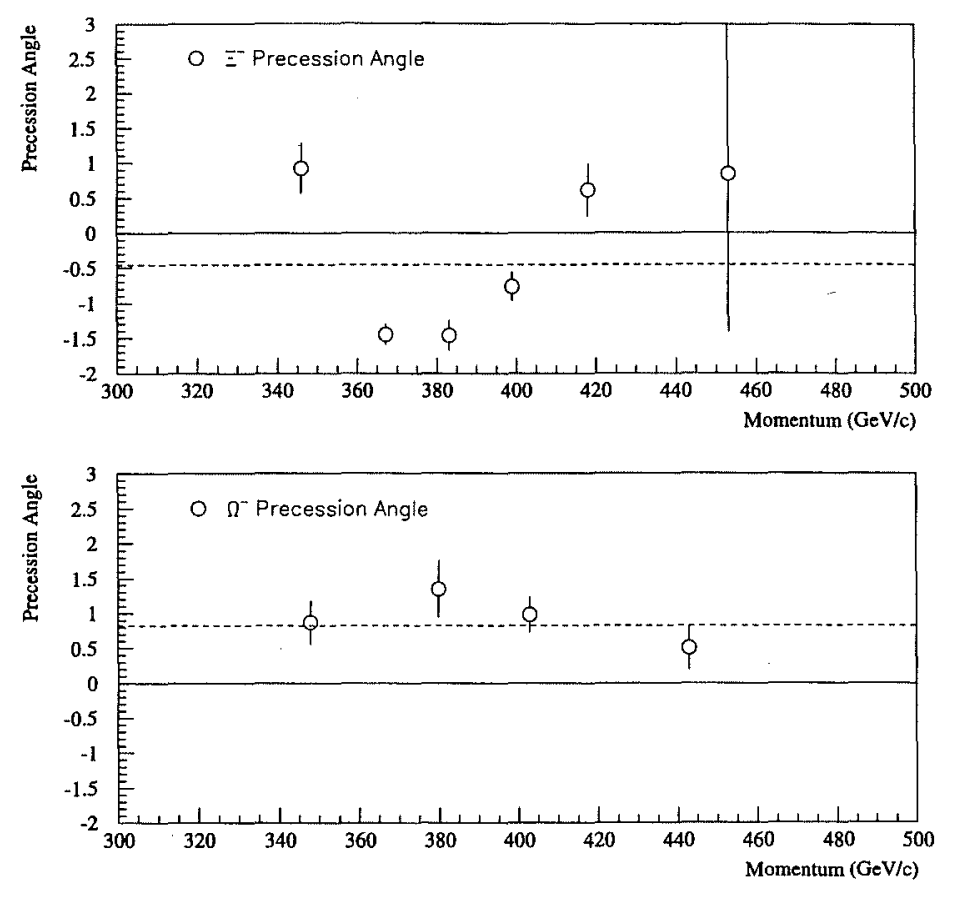

Figure 5.5: Measured precession angles for the unpolarized neutral beam production sample with the sweeper magnet set at -2900 Amps. The dashed lines show the expected precession angles calculated using the known magnetic moments. 


\begin{tabular}{|c|c|c|c|c|}
\hline $\begin{array}{c}\text { Mom. } \\
(\mathrm{GeV} / \mathrm{c})\end{array}$ & $\begin{array}{c}\text { Number of } \\
\text { Events }(1000 \mathrm{~s})\end{array}$ & View & $\begin{array}{c}\text { Asymmetry } \\
\alpha_{\Lambda} \gamma_{\Xi} P \Xi \\
\end{array}$ & Bias \\
\hline \multirow{3}{*}{347.} & \multirow{3}{*}{93} & $\mathrm{x}$ & $-0.0395 \pm 0.0061$ & $0.0126 \pm 0.0061$ \\
\hline & & $\mathrm{y}$ & $-0.0149 \pm 0.0068$ & $0.0496 \pm 0.0068$ \\
\hline & & $z$ & $0.0168 \pm 0.0078$ & $-0.0849 \pm 0.0078$ \\
\hline \multirow{3}{*}{367.} & \multirow{3}{*}{125} & $\mathrm{x}$ & $-0.0526 \pm 0.0049$ & $-0.0036 \pm 0.0049$ \\
\hline & & y & $0.0006 \pm 0.0057$ & $0.0297 \pm 0.0057$ \\
\hline & & $\mathrm{z}$ & $0.0261 \pm 0.0062$ & $-0.0117 \pm 0.0062$ \\
\hline \multirow{3}{*}{383.} & \multirow{3}{*}{138} & $\mathrm{x}$ & $-0.0671 \pm 0.0046$ & $-0.0121 \pm 0.0046$ \\
\hline & & $y$ & $0.0030 \pm 0.0054$ & $0.0102 \pm 0.0054$ \\
\hline & & $z$ & $0.0306 \pm 0.0059$ & $-0.0089 \pm 0.0059$ \\
\hline \multirow{3}{*}{399.} & \multirow{3}{*}{129} & $\mathrm{x}$ & $-0.0557 \pm 0.0048$ & $-0.0050 \pm 0.0048$ \\
\hline & & $\mathrm{y}$ & $0.0092 \pm 0.0056$ & $0.0035 \pm 0.0056$ \\
\hline & & $\mathrm{z}$ & $0.0232 \pm 0.0062$ & $0.0086 \pm 0.0062$ \\
\hline \multirow{3}{*}{417.} & \multirow{3}{*}{130} & $\bar{x}$ & $-0.0695 \pm 0.0048$ & $-0.0136 \pm 0.0048$ \\
\hline & & $\mathrm{y}$ & $-0.0024 \pm 0.0056$ & $0.0002 \pm 0.0056$ \\
\hline & & $z$ & $0.0352 \pm 0.0062$ & $0.0125 \pm 0.0062$ \\
\hline \multirow{3}{*}{451.} & \multirow{3}{*}{96} & $\mathrm{x}$ & $-0.0797 \pm 0.0060$ & $-0.0186 \pm 0.0060$ \\
\hline & & y & $-0.0025 \pm 0.0065$ & $0.0153 \pm 0.0065$ \\
\hline & & $\mathrm{z}$ & $0.0592 \pm 0.0075$ & $0.0377 \pm 0.0075$ \\
\hline
\end{tabular}

Table 5.7: Measured $\Xi^{-}$asymmetries and biases as a function of momentum for the polarized neutral beam production sample.

\begin{tabular}{c|c|c|c|c}
$\begin{array}{c}\text { Mom. } \\
(\mathrm{GeV} / \mathrm{c})\end{array}$ & $\begin{array}{c}\text { Number of } \\
\text { Events }(1000 \mathrm{~s})\end{array}$ & View & $\begin{array}{c}\text { Asymmetry } \\
\alpha_{\Lambda} P_{\Omega}\end{array}$ & Bias \\
\hline 351. & 5 & $\mathrm{x}$ & $-0.0351 \pm 0.0277$ & $-0.0338 \pm 0.0277$ \\
& & $\mathrm{y}$ & $0.0207 \pm 0.0295$ & $0.0699 \pm 0.0295$ \\
& & $\mathrm{z}$ & $-0.0596 \pm 0.0354$ & $0.0212 \pm 0.0354$ \\
\hline & & $\mathrm{x}$ & $-0.0100 \pm 0.0242$ & $0.0215 \pm 0.0242$ \\
380. & 5 & $\mathrm{y}$ & $-0.0299 \pm 0.0276$ & $0.0218 \pm 0.0276$ \\
& & $\mathrm{z}$ & $0.0103 \pm 0.0290$ & $0.0516 \pm 0.0290$ \\
\hline & & $\mathrm{x}$ & $-0.0146 \pm 0.0261$ & $0.0196 \pm 0.0261$ \\
403. & 4 & $\mathrm{y}$ & $-0.0022 \pm 0.0290$ & $-0.0648 \pm 0.0290$ \\
& & $\mathrm{z}$ & $-0.0807 \pm 0.0309$ & $0.0386 \pm 0.0309$ \\
\hline & & $\mathrm{x}$ & $-0.0678 \pm 0.0273$ & $0.0079 \pm 0.0273$ \\
439. & 4 & $\mathrm{y}$ & $0.0312 \pm 0.0285$ & $-0.0677 \pm 0.0285$ \\
& & $\mathrm{z}$ & $-0.0082 \pm 0.0314$ & $0.0517 \pm 0.0314$ \\
\hline
\end{tabular}

Table 5.8: Measured $\Omega^{-}$asymmetries and biases as a function of momentum for the polarized neutral beam production sample.

\begin{tabular}{c|c|c|c|c|c}
$\begin{array}{c}\text { Sweeper } \\
\text { Current }\end{array}$ & $\begin{array}{c}\text { Mom. } \\
(\mathrm{GeV} / \mathrm{c})\end{array}$ & $\begin{array}{c}\text { Number of } \\
\text { Events (1000s) }\end{array}$ & View & $\begin{array}{c}\text { Asymmetry } \\
\alpha_{\Lambda} \gamma \equiv P \Xi\end{array}$ & Bias \\
\hline & & & $\mathrm{x}$ & $-0.0605 \pm 0.0021$ & $-0.0072 \pm 0.0021$ \\
2900 & 393. & 711 & $\mathrm{y}$ & $-0.0004 \pm 0.0024$ & $0.0165 \pm 0.0024$ \\
Amps & & & $\mathrm{z}$ & $0.0300 \pm 0.0027$ & $-0.0071 \pm 0.0027$ \\
\hline
\end{tabular}

Table 5.9: Overall $\Xi^{-}$sample asymmetries and biases for the polarized neutral beam production sample.

\begin{tabular}{c|c|c|c|c|c}
$\begin{array}{c}\text { Sweeper } \\
\text { Current }\end{array}$ & $\begin{array}{c}\text { Mom. } \\
(\mathrm{GeV} / \mathrm{c})\end{array}$ & $\begin{array}{c}\text { Number of } \\
\text { Events (1000s) }\end{array}$ & View & $\begin{array}{c}\text { Asymmetry } \\
\alpha_{\Lambda} P_{\Omega}\end{array}$ & Bias \\
\hline & & & $\mathrm{x}$ & $-0.0283 \pm 0.0132$ & $0.0096 \pm 0.0132$ \\
2900 & 393. & 18 & $\mathrm{y}$ & $0.0066 \pm 0.0144$ & $-0.0084 \pm 0.0144$ \\
Amps & & & $\mathrm{z}$ & $-0.0341 \pm 0.0159$ & $0.0428 \pm 0.0159$ \\
\hline
\end{tabular}

Table 5.10: Overall $\Omega^{-}$sample asymmetries and biases for the polarized neutral beam production sample.

\begin{tabular}{c|c|c|c|c|c}
$\begin{array}{c}\text { Neutral Beam } \\
\text { Type }\end{array}$ & $\begin{array}{c}\text { Sweeper } \\
\text { Current }\end{array}$ & Particle & $\begin{array}{c}\text { Number of } \\
\text { Events }(1000 \mathrm{~s})\end{array}$ & $\begin{array}{c}\text { Mom. } \\
(\mathrm{GeV} / \mathrm{c})\end{array}$ & Polarization \\
\hline Polarized & -2900 & $\Xi^{-}$ & 711 & 393. & $-0.118 \pm 0.004$ \\
& Amps & $\Omega^{-}$ & 18 & 393. & $-0.069 \pm 0.023$ \\
\hline Unpolarized & -750 & $\Xi^{-}$ & 3768 & 300. & $-0.006 \pm 0.001$ \\
& Amps & $\Omega^{-}$ & 50 & 304. & $+0.036 \pm 0.015$ \\
& -2900 & $\Xi^{-}$ & 9971 & 395. & $-0.003 \pm 0.001$ \\
& Amps & $\Omega^{-}$ & 167 & 394. & $+0.044 \pm 0.008$ \\
\hline
\end{tabular}

Table 5.11: Fitted target polarizations for all data samples. 

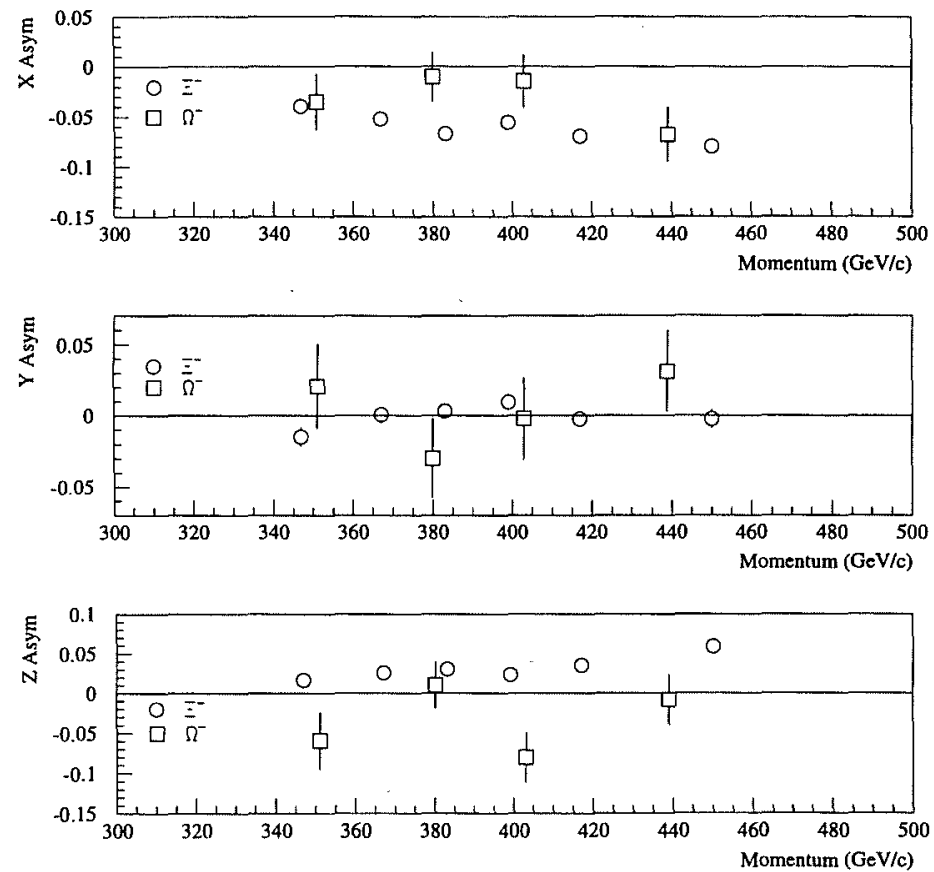

Figure 5.6: Asymmetries $\left(\alpha_{\Lambda} \gamma_{\Xi} P_{\Xi}\right.$ and $\alpha_{\Lambda} P_{\Omega}$ ) for the polarized neutral beam production sample.
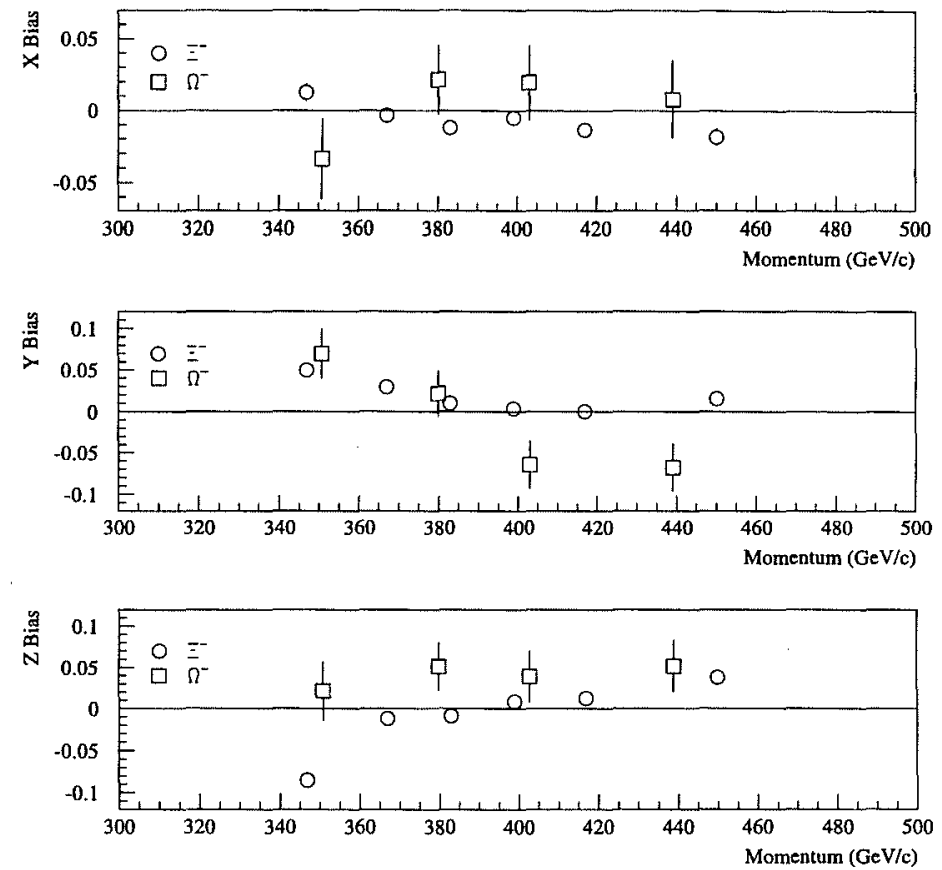

Figure 5.7: Biases for the polarized neutral beam production sample. 

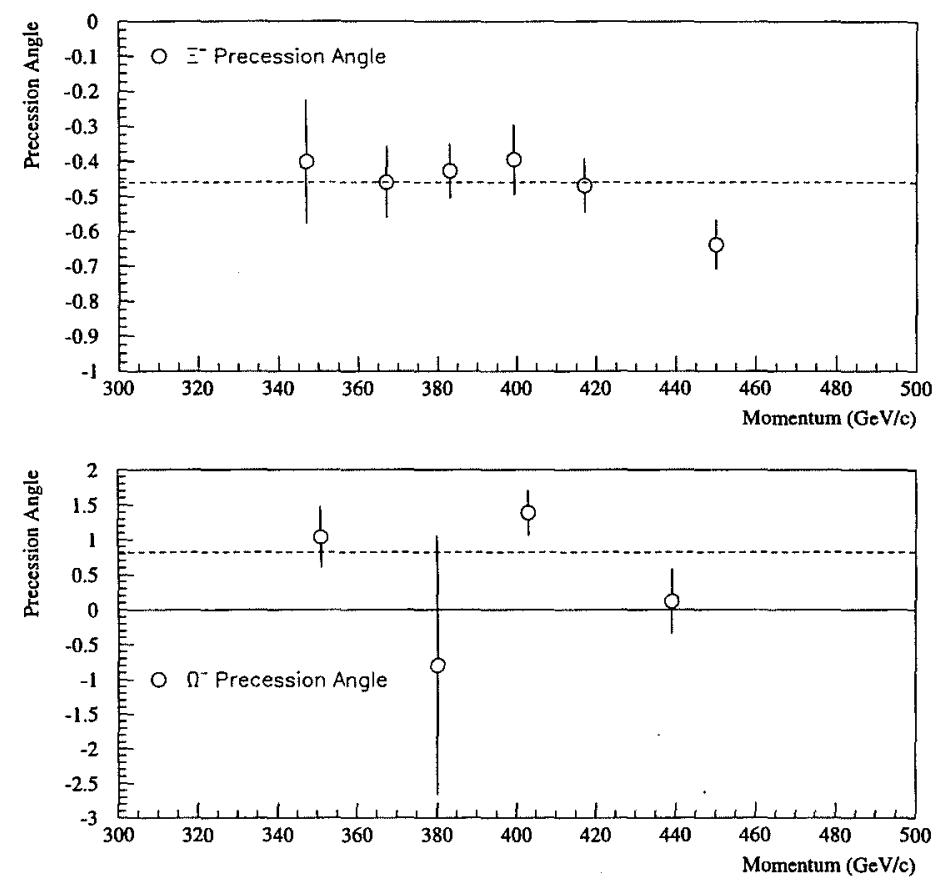

Figure 5.8: Measured precession angles for the polarized neutral beam production sample. The dashed lines show the expected precession angles calculated using the known magnetic moments.

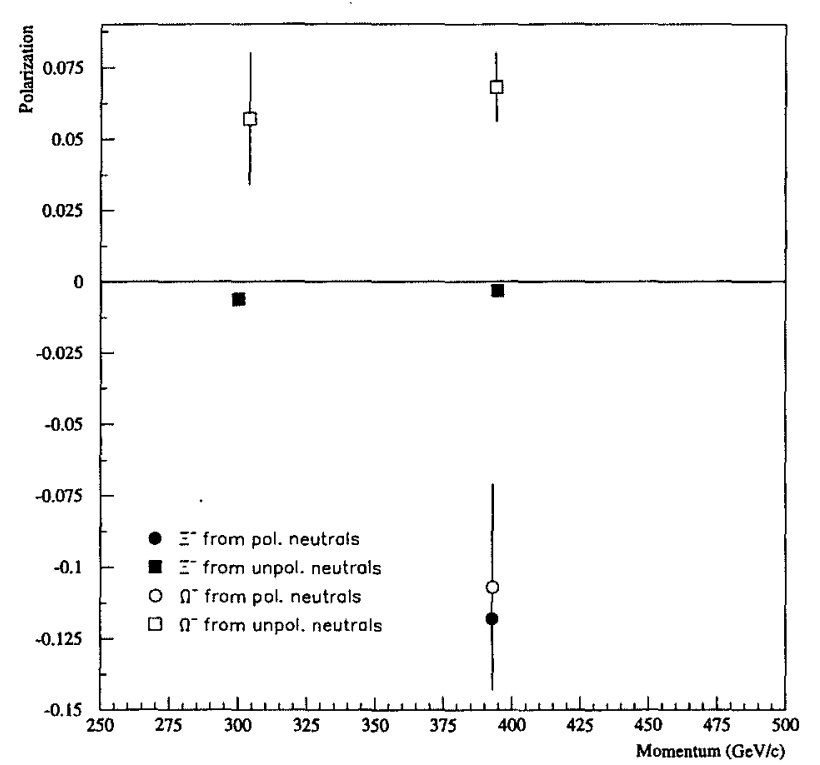

Figure 5.9: Fitted target polarizations for all samples. 
amps in the Hyperon magnet are consistent with each other. This is expected since both samples cover the same momentum region. Also, it can be seen that, with the exception of the the lowest momentum point in the $x$ view for the $750 \mathrm{amp}$ sample, the biases for the two unpolarized neutral beam samples show a similar behavior, as expected. The biases for these two samples are not the same in the momentum region where they overlap. This is due to the fact that while the data from the two samples have the same momentum, the particles in the two samples pass through different regions of the charged collimator and the spectrometer.

The biases for the $\Xi^{-}$and $\Omega^{-}$samples can be compared in Figures 5.2, 5.4, and 5.7. These figures show that while the $\Xi^{-}$and $\Omega^{-}$biases have similar momentum dependences, their magnitudes are different. This difference is due to the different parent and daughter particle masses. Due to these mass differences, the daughter particles from the two decays have a different range of allowed opening angles in the laboratory. This means that the daughter particles from the two decays will have different distributions in the various parts of the spectrometer.

The large magnitude of the biases, especially in the low momentum region of the $z$ view caused some concern. It was found that the data selection criteria could be varied to reduce these biases. However, reducing the biases had no effect on the asymmetry results.

Monte Carlo studies were performed using both polarized and unpolarized Monte Carlo data, and it was found the biases observed in the Monte Carlo analysis were in general similar to those of real data. In addition, the asymmetry results for the unpolarized Monte Carlo data adequately matched the asymmetry results of the unpolarized neutral beam data sample. The results of the asymmetry analysis of the polarized Monte Carlo data were consistent with the input polarization used in the generation of the Monte Carlo data.
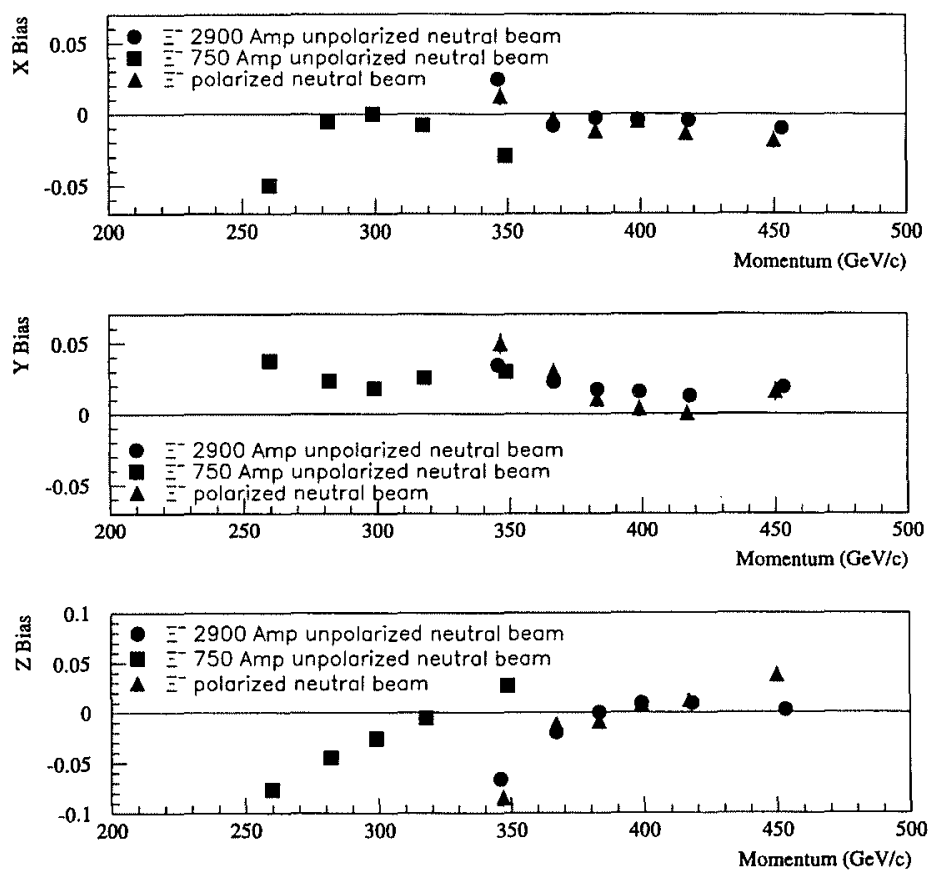

Figure 5.10: Comparision of biases for all the $\Xi^{-}$samples. 


\subsection{Systematic Studies}

\subsubsection{Single-track Triggers}

The effect of requiring hits in C11 and C12 in the trigger was studied by analyzing $\Xi^{-}$ events that were only required to have hits in the scintillation counters S1 and S2 with no hits in V1 or V2. This trigger selected events that were from charged particles that had entered the spectrometer from the Hyperon magnet and were not part of a shower from an upstream interaction. Table 5.12 gives the measured asymmetries $\left(\alpha_{\Lambda} \gamma_{\Xi} P_{\Xi}\right)$ and biases as a function of momentum for $\Xi^{-}$events selected by the single-track trigger. These events are from the unpolarized neutral beam production data sample taken with a current of -2900 amps in the Hyperon magnet.

Figures 5.11 and 5.12 show the unpolarized neutral beam production asymmetries and biases plotted as a function of momentum for the $\Xi^{-}$events selected by the singletrack trigger. The distributions for events from the general unpolarized neutral beam production sample are shown for comparison. All of these distributions agree within the statistical errors of the measurements. As a further test, the mass, momentum, $\Xi^{-}$ and $\Lambda$ vertex, target position, $\chi^{2}{ }_{G} / D F$ and $\chi^{2}{ }_{\Lambda}$ distributions of the events selected by the single-track trigger were compared to the distributions for events selected by the three-track trigger, and no significant differences were found.

\subsubsection{Data at 0 mrad production angle.}

Table 5.13 gives the measured asymmetries $\left(\alpha_{\Lambda} \gamma \Xi P \Xi\right)$ and biases as a function of momentum for data produced with no production angle. This data was collected with two different magnetic field polarities in the neutral sweeper magnet and this division of the sample was used in the bias cancellation instead of a positive and negative production angle division. Figures 5.13 and 5.14 show the $\Xi^{-}$asymmetries and biases plotted as a function of momentum for the 0 mrad production angle data.

\begin{tabular}{c|c|c|c|c}
$\begin{array}{c}\text { Mom. } \\
(\mathrm{GeV} / \mathrm{c})\end{array}$ & $\begin{array}{c}\text { Number of } \\
\text { Events (1000s) }\end{array}$ & View & $\begin{array}{c}\text { Asymmetry } \\
\alpha_{\Lambda} \gamma_{\Xi} P_{\Xi}\end{array}$ & Bias \\
\hline 346. & \multirow{2}{*}{19} & $\mathrm{x}$ & $-0.0148 \pm 0.0134$ & $-0.0405 \pm 0.0134$ \\
& & $\mathrm{y}$ & $0.0148 \pm 0.0143$ & $0.0097 \pm 0.0143$ \\
$\mathrm{z}$ & $-0.0170 \pm 0.0169$ & $-0.0316 \pm 0.0169$ \\
\hline & & $\mathrm{x}$ & $-0.0013 \pm 0.0115$ & $-0.0283 \pm 0.0115$ \\
367. & 22 & $\mathrm{y}$ & $-0.0119 \pm 0.0129$ & $0.0175 \pm 0.0129$ \\
& & $\mathrm{z}$ & $-0.0080 \pm 0.0141$ & $-0.0253 \pm 0.0141$ \\
\hline & & $\mathrm{x}$ & $-0.0093 \pm 0.0110$ & $0.0119 \pm 0.0110$ \\
383. & 25 & $\mathrm{y}$ & $0.0019 \pm 0.0121$ & $0.0204 \pm 0.0121$ \\
& & $\mathrm{z}$ & $-0.0348 \pm 0.0136$ & $0.0083 \pm 0.0136$ \\
\hline & & $\mathrm{x}$ & $0.0066 \pm 0.0114$ & $0.0130 \pm 0.0114$ \\
399. & 24 & $\mathrm{y}$ & $-0.0023 \pm 0.0125$ & $-0.0077 \pm 0.0125$ \\
& & $\mathrm{z}$ & $-0.0152 \pm 0.0142$ & $0.0107 \pm 0.0142$ \\
\hline & & $\mathrm{x}$ & $0.0279 \pm 0.0108$ & $0.0063 \pm 0.0108$ \\
418. & 26 & $\mathrm{y}$ & $0.0144 \pm 0.0118$ & $0.0031 \pm 0.0118$ \\
& & $\mathrm{z}$ & $0.0167 \pm 0.0135$ & $-0.0115 \pm 0.0135$ \\
\hline \multirow{3}{*}{456.} & \multirow{2}{*}{25} & $\mathrm{x}$ & $0.0086 \pm 0.0118$ & $-0.0352 \pm 0.0118$ \\
& & $\mathrm{y}$ & $0.0037 \pm 0.0122$ & $0.0092 \pm 0.0122$ \\
& & $\mathrm{z}$ & $0.0083 \pm 0.0145$ & $0.0297 \pm 0.0145$ \\
\hline
\end{tabular}

Table 5.12: Measured $\Xi^{-}$asymmetries and biases as a function of momentum for the single-track unpolarized neutral beam production sample with the sweeper magnet set at -2900 amps 

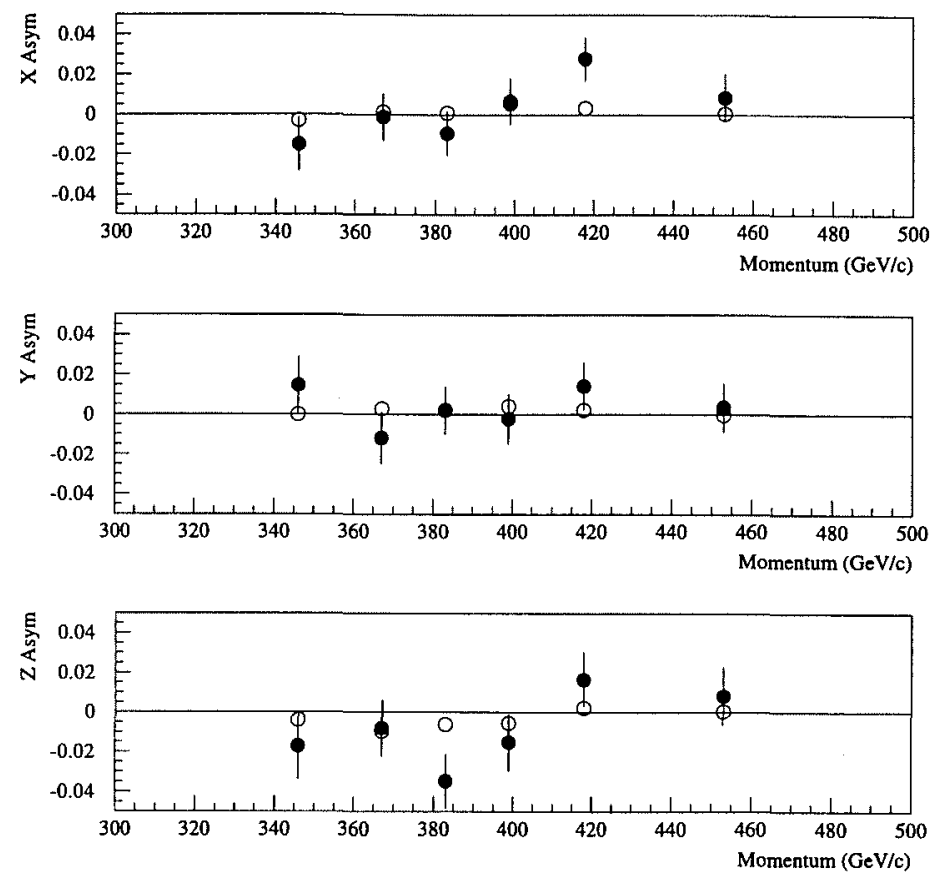

Figure 5.11: Comparison of $\Xi^{-}$asymmetries $\left(\alpha_{\Lambda} \gamma_{\Xi} P \Xi\right)$ for the normal trigger (open circles) and single-track trigger (filled circles)
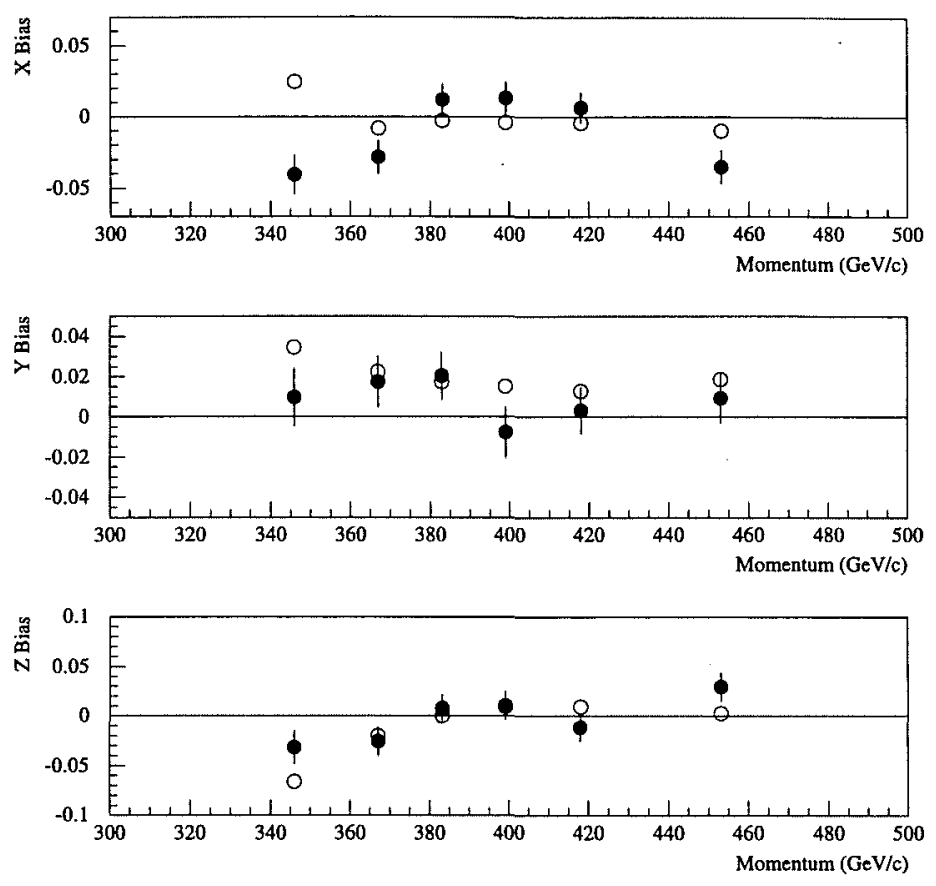

Figure 5.12: Comparison of $\Xi^{-}$biases for the normal trigger (open circles) and singletrack trigger (filled circles). 


\begin{tabular}{|c|c|c|c|c|}
\hline $\begin{array}{c}\text { Mom. } \\
(\mathrm{GeV} / \mathrm{c})\end{array}$ & $\begin{array}{c}\text { Number of } \\
\text { Events }(1000 \mathrm{~s})\end{array}$ & View & $\begin{array}{c}\text { Asymmetry } \\
\alpha_{\Lambda} \gamma_{\Xi} P_{\Xi}\end{array}$ & Bias \\
\hline \multirow{3}{*}{347.} & \multirow{3}{*}{124} & $\mathbf{x}$ & $-0.0018 \pm 0.0053$ & $0.0282 \pm 0.0053$ \\
\hline & & $\mathrm{y}$ & $0.0103 \pm 0.0056$ & $0.0472 \pm 0.0056$ \\
\hline & & $z$ & $0.0072 \pm 0.0067$ & $-0.0816 \pm 0.0067$ \\
\hline \multirow{3}{*}{367.} & \multirow{3}{*}{172} & $\mathrm{x}$ & $-0.0102 \pm 0.0042$ & $-0.0035 \pm 0.0042$ \\
\hline & & $y$ & $0.0014 \pm 0.0047$ & $0.0258 \pm 0.0047$ \\
\hline & & $\mathbf{z}$ & $0.0041 \pm 0.0052$ & $-0.0235 \pm 0.0052$ \\
\hline \multirow{3}{*}{383.} & \multirow{3}{*}{206} & $\bar{x}$ & $0.0006 \pm 0.0038$ & $0.0028 \pm 0.0038$ \\
\hline & & $\mathrm{y}$ & $0.0020 \pm 0.0042$ & $0.0140 \pm 0.0042$ \\
\hline & & $\mathrm{z}$ & $0.0089 \pm 0.0048$ & $0.0013 \pm 0.0048$ \\
\hline \multirow{3}{*}{399.} & \multirow{3}{*}{209} & $\mathrm{x}$ & $-0.0021 \pm 0.0038$ & $-0.0058 \pm 0.0038$ \\
\hline & & $\mathrm{y}$ & $-0.0012 \pm 0.0042$ & $0.0124 \pm 0.0042$ \\
\hline & & $z$ & $0.0049 \pm 0.0048$ & $0.0068 \pm 0.0048$ \\
\hline \multirow{3}{*}{418.} & \multirow{3}{*}{243} & $\mathrm{x}$ & $-0.0010 \pm 0.0036$ & $0.0003 \pm 0.0036$ \\
\hline & & $y$ & $0.0030 \pm 0.0038$ & $0.0080 \pm 0.0038$ \\
\hline & & $z$ & $0.0037 \pm 0.0044$ & $0.0055 \pm 0.0044$ \\
\hline \multirow{3}{*}{455.} & \multirow{3}{*}{245} & $\mathrm{x}$ & $-0.0005 \pm 0.0038$ & $-0.0052 \pm 0.0038$ \\
\hline & & $y$ & $0.0014 \pm 0.0039$ & $0.0083 \pm 0.0039$ \\
\hline & & $z$ & $0.0070 \pm 0.0047$ & $0.0241 \pm 0.0047$ \\
\hline
\end{tabular}

Table 5.13: Measured $\Xi^{-}$asymmetries and biases as a function of momentum for data with no production angle.

To study how well the polarization analysis measured unpolarized data samples, Monte Carlo $\Xi^{-}$data generated with no polarization was analyzed. Figure 5.15 shows the results of this analysis, with the measured asymmetries for the unpolarized neutral beam production data for comparison. This figure shows that the measured asymmetries for the Monte Carlo data are consistent with no polarization. The agreement between the real data and Monte Carlo asymmetries also add support to our conclusion that the $\Xi^{-} \mathrm{s}$ produced by the unpolarized neutral beam are unpolarized.

\subsubsection{Other Systematic Studies}

To search for possible time dependent effects in the data, the unpolarized neutral beam production $\Xi^{-}$data sample with sweeper current set at -2900 amps was broken into
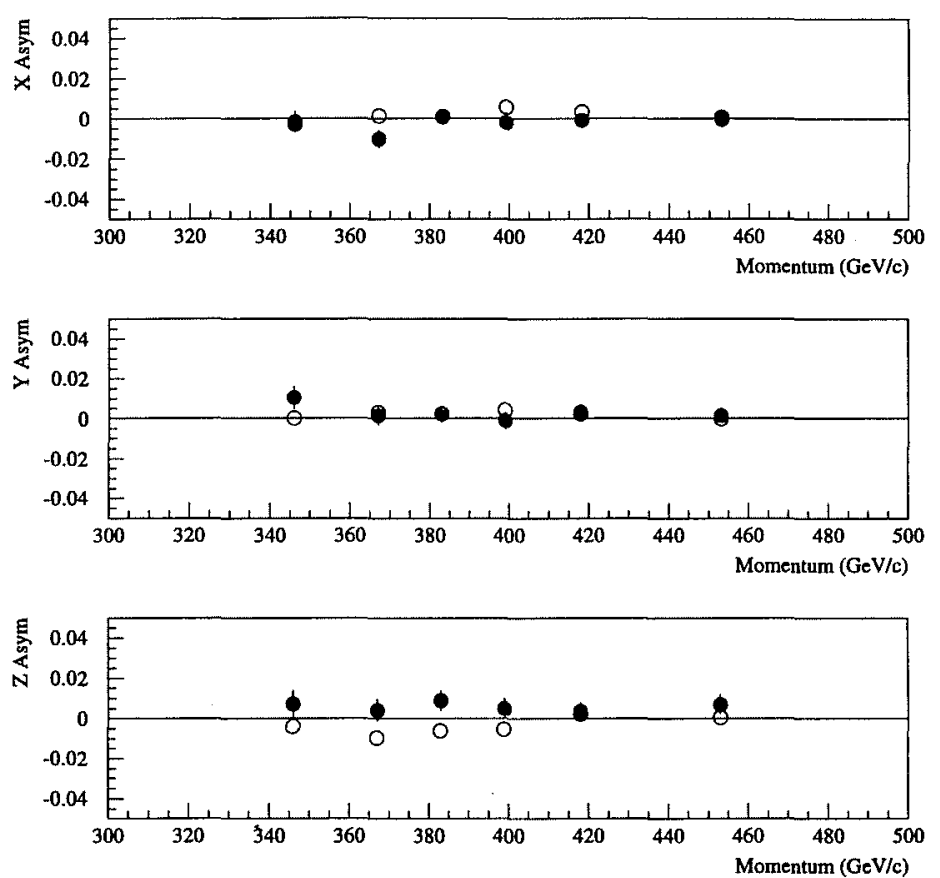

Figure 5.13: Comparison of $\Xi^{-}$asymmetries $\left(\alpha_{\Lambda} \gamma_{\Xi} P \Xi\right)$ for the unpolarized neutral beam production data (open circles) and 0 mrad production data (filled circles). 

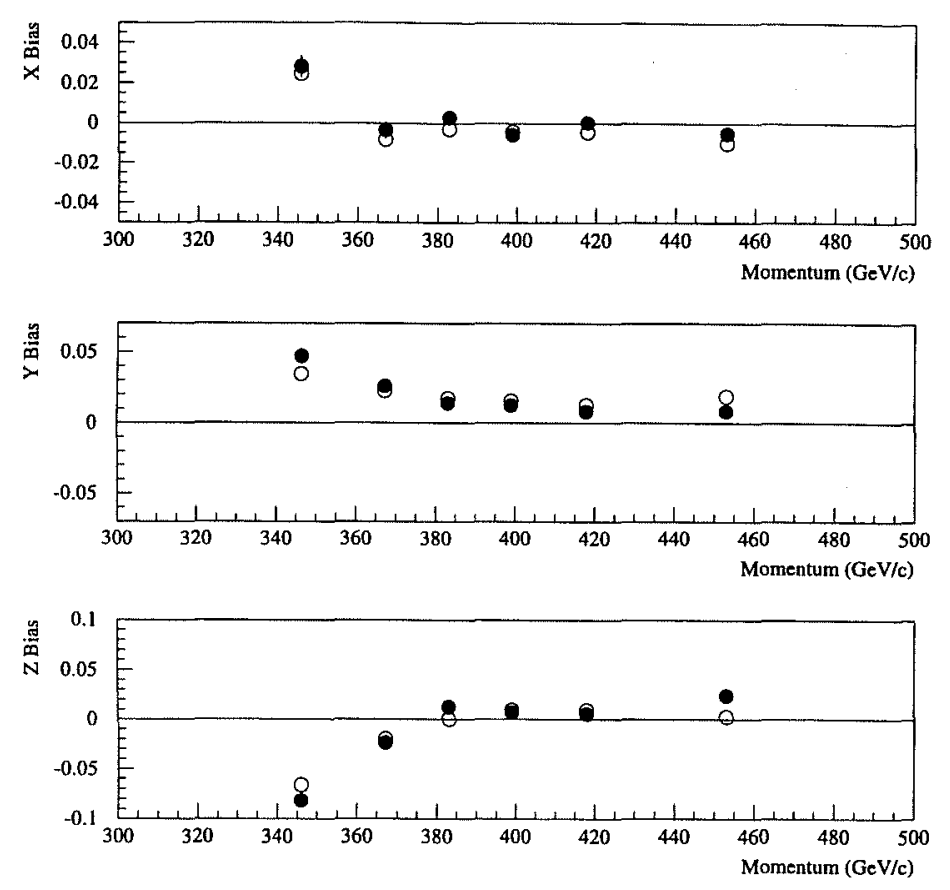

Figure 5.14: Comparison of $\Xi^{-}$biases for the unpolarized neutral beam production data (open circles) and 0 mrad production data (filled circles).
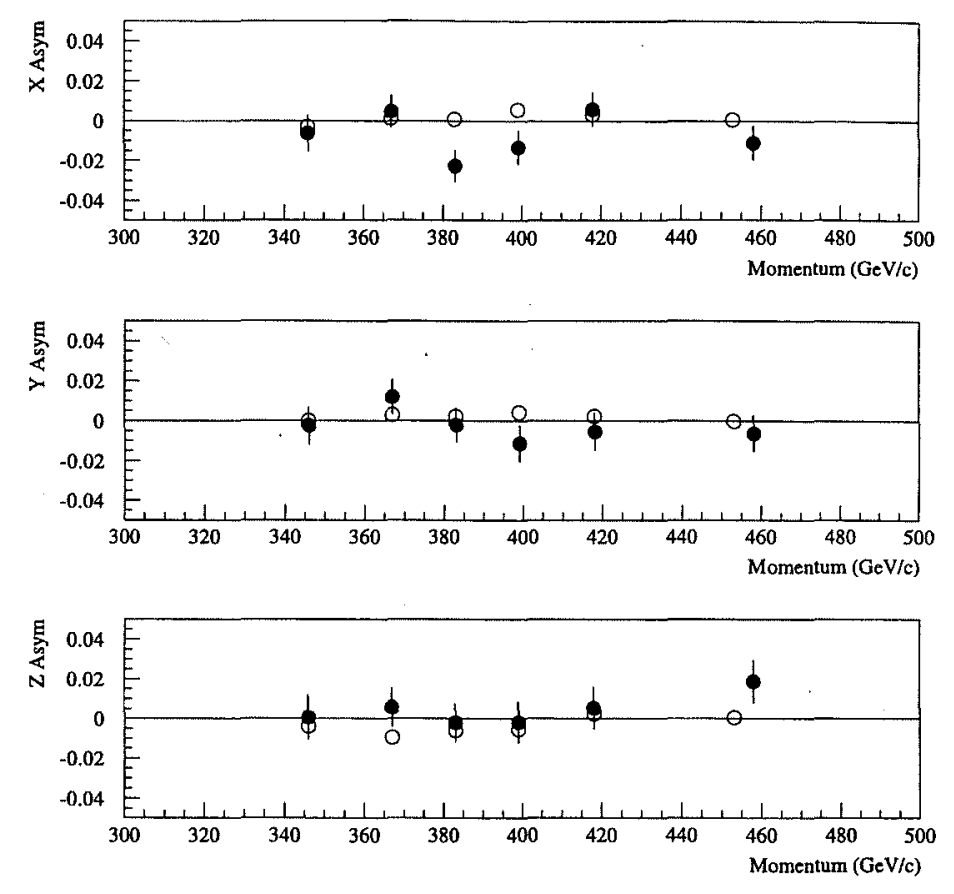

Figure 5.15: Comparison of $\Xi^{-}$asymmetries $\left(\alpha_{\Lambda} \gamma \Xi P \Xi\right)$ for the unpolarized neutral beam production data (open circles) and unpolarized Monte Carlo data (filled circles). 


\begin{tabular}{c|c} 
Normal Criteria & "Tighter" Criteria \\
\hline$\Delta m_{\Xi^{-}}<15 \mathrm{MeV} / \mathrm{c}$ & $\Delta m_{\Xi^{-}}<8 \mathrm{MeV} / \mathrm{c}$ \\
\hline$\chi_{G}^{2} / D F<4$ & $\chi_{G}^{2} / D F<3$ \\
$\chi_{\Lambda}^{2}<15$ & $\chi_{\Lambda}^{2}<10$ \\
\hline$\left|X_{T}\right|<0.9 \mathrm{~cm}$ & $\left|X_{T}\right|<0.5 \mathrm{~cm}$ \\
$\left|Y_{T}\right|<0.8 \mathrm{~cm}$ & $\left|Y_{T}\right|<0.5 \mathrm{~cm}$ \\
\hline maximum $Z_{\Xi^{-}}=2500 \mathrm{~cm}$ & maximum $Z_{\Xi^{-}}=2000 \mathrm{~cm}$ \\
minimum $Z_{\Xi^{-}}=-1000 \mathrm{~cm}$ & minimum $Z_{\Xi^{-}}=+200 \mathrm{~cm}$ \\
\hline
\end{tabular}

Table 5.14: Normal and "tighter" data selection criteria.

seven smaller parts. The subdivision was based on the point in the run the data were taken. Figures 5.16 and 5.17 show the measured asymmetries and biases for the seven subsamples. There is no evidence of a time dependent systematic effect.

As a final systematic study, the effect of variations in the data selection criteria were studied. This was done by studying the effect of tighter selection criteria on the $\Xi^{-}$ magnetic moment measured for the polarized neutral beam data sample. Table 5.14 shows the normal and tighter data selection criteria that were used in this study. The effects of varying each selection criteria were studied individually. Figure 5.18 shows the measured $\Xi^{-}$magnetic moment as a function of momentum for the different selection criteria. The shaded region represents the world average measurement of $\mu_{\Xi-}$ and its measured error [40]. There is no evidence of any systematic effect due to the data selection method.

\subsection{Summary of Results}

To summarize, the polarization of $\Xi^{-} \mathrm{s}$ and $\Omega^{-}$produced by an unpolarized neutral beam has been has been measured for the first time. The $\Xi^{-} \mathrm{s}$ were found to be unpolarized. The observed $\Omega^{-}$polarization was $+0.044 \pm 0.008$ at an average momentum of 394. $\mathrm{GeV} / \mathrm{c}$ and $+0.036 \pm 0.015$ at an average momentum of $305 . \mathrm{GeV} / \mathrm{c}$.

The polarization of $\Xi^{-} s$ and $\Omega^{-} s$ produced by a polarized neutral beam was also
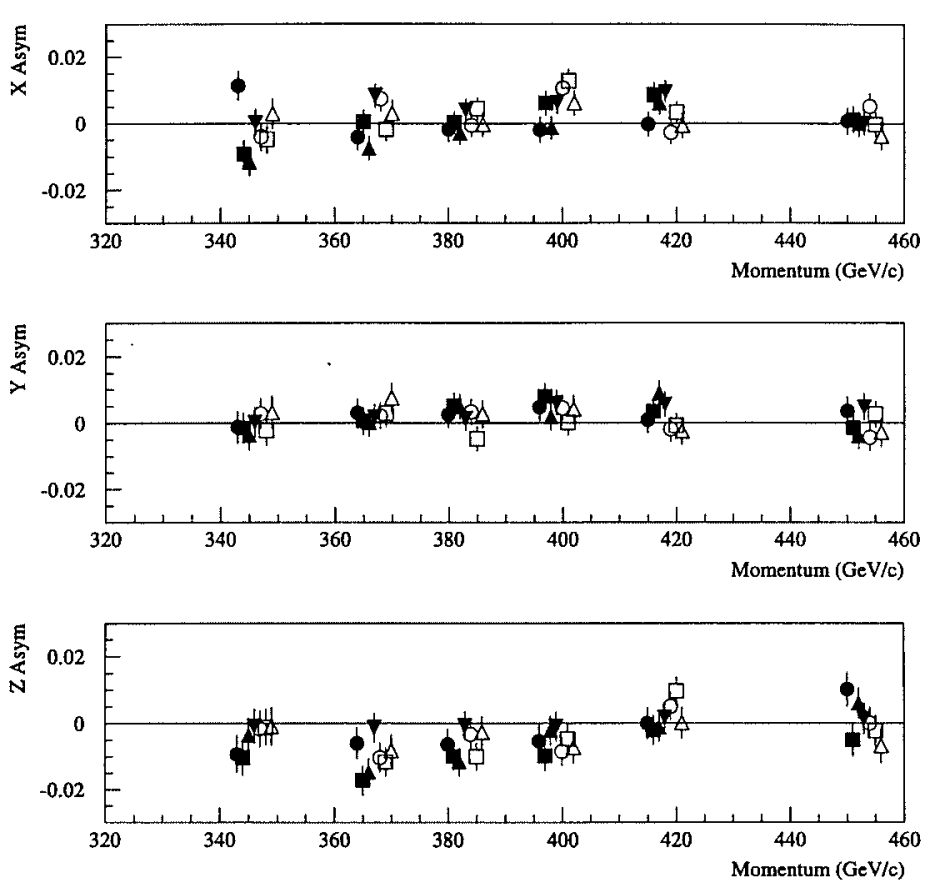

Figure 5.16: $\Xi^{-}$asymmetries $\left(\alpha_{\Lambda} \gamma \Xi P \Xi\right)$ for seven unpolarized neutral beam production data subsamples. 

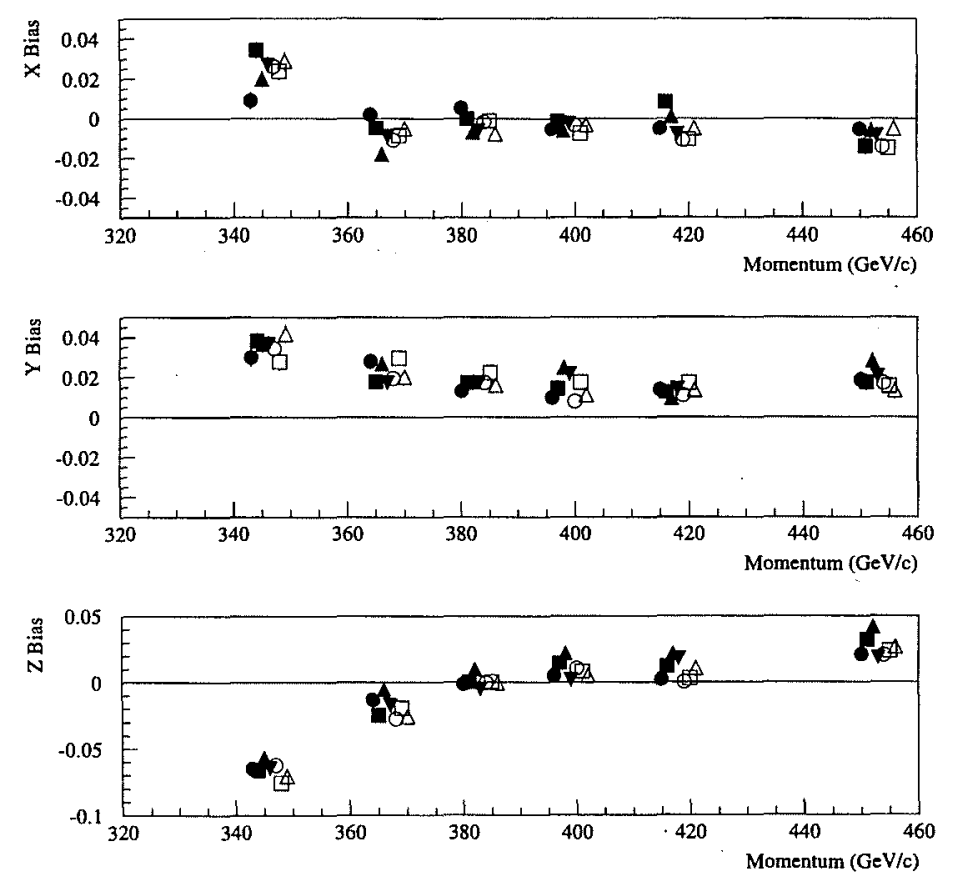

Figure 5.17: $\Xi^{-}$biases for seven unpolarized neutral beam production data subsamples.

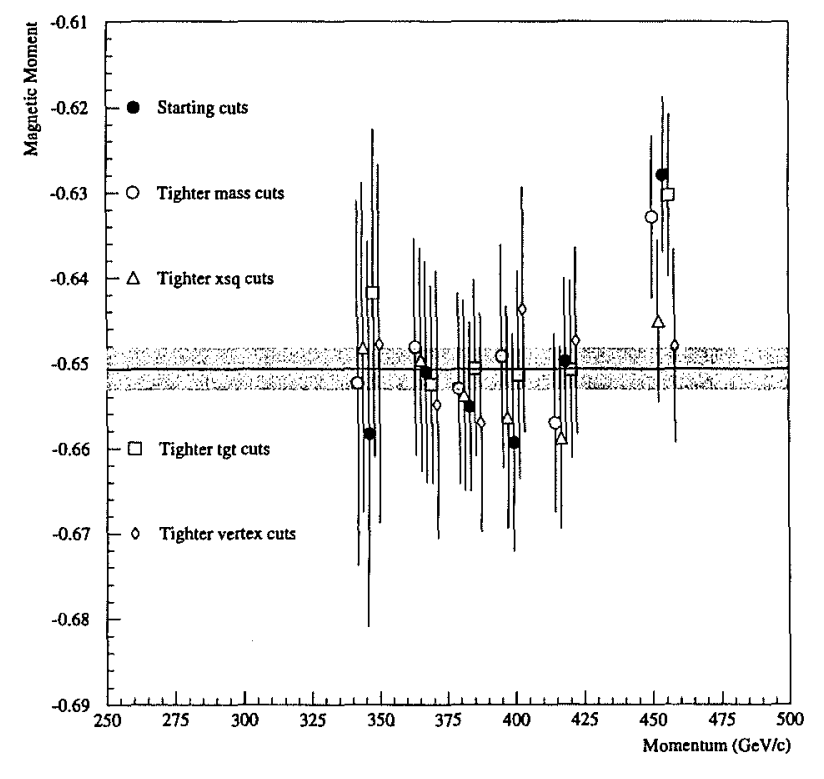

Figure 5.18: Measured $\Xi^{-}$magnetic moment for various "tighter" data selection criteria. 
studied. The $\Xi^{-}$sample had a polarization of $-0.118 \pm 0.004$ at an average momentum of 393 . GeV/c. The $\Omega^{-}$sample had a polarization of $-0.069 \pm 0.023$ at an average momentum of $394 . \mathrm{GeV} / \mathrm{c}$.
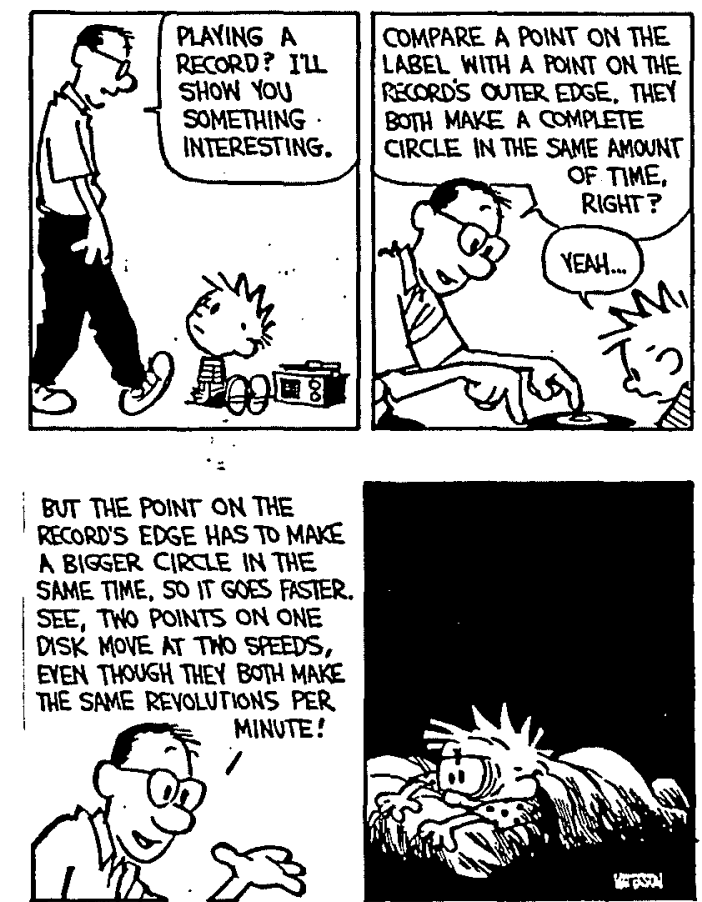

CALVIN AND HOBBES copyright Watterson. Dist. by UNIVERSAL PRESS SYNDICATE. Reprinted with permission. All rights reserved. 


\section{Chapter 6}

\section{Conclusions}

\subsection{Comparision With Previous Measurements}

The measured $\Xi^{-}$and $\Omega^{-}$polarizations at the production target are show in Figures 6.1 and 6.2 for both the polarized and unpolarized neutral beam production modes. Results for a previous measurement using the polarized neutral beam production mode are shown for comparison [26]. These figures show that the polarized neutral beam production mode results discussed in this thesis are consistent with the previous measurement.

\subsection{Theoretical Predictions}

Of the models discussed in Chapter 1 , only two are able to make predictions of the polarizations that were studied in this experiment. The Lund model of Andersson, Gustafson, and Ingelman[33] predicts that all $s$ quarks produced from the sea should be polarized in the negative direction. With this prediction for the $s$ quark polarization, one expects that $\Omega^{-}$s produced from an unpolarized neutral beam would be polarized with a negative sign. This does not agree with the experimental observation of a positive

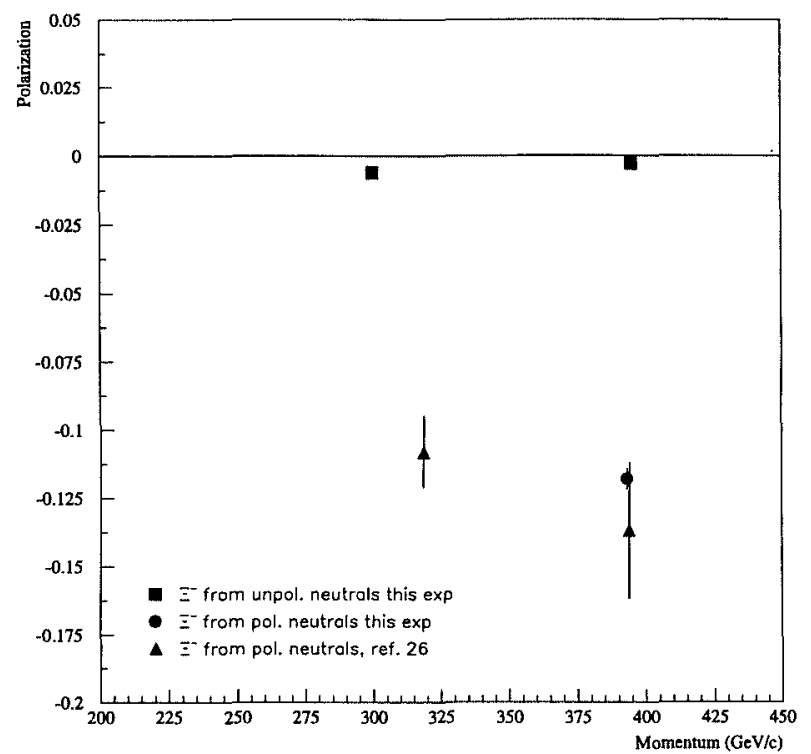

Figure 6.1: Comparision of $\Xi^{-}$polarization results. 
polarization.

The model of DeGrand and Miettinen [29] makes more detailed predictions of the polarization of hyperons produced from both a polarized and an unpolarized neutral beam. The predictions of this model with the modification introduced by Bonner et al. [21] to allow for quark spin flips are given in Table 1.3 .

For production from the unpolarized neutral beam, we set the incident beam po-

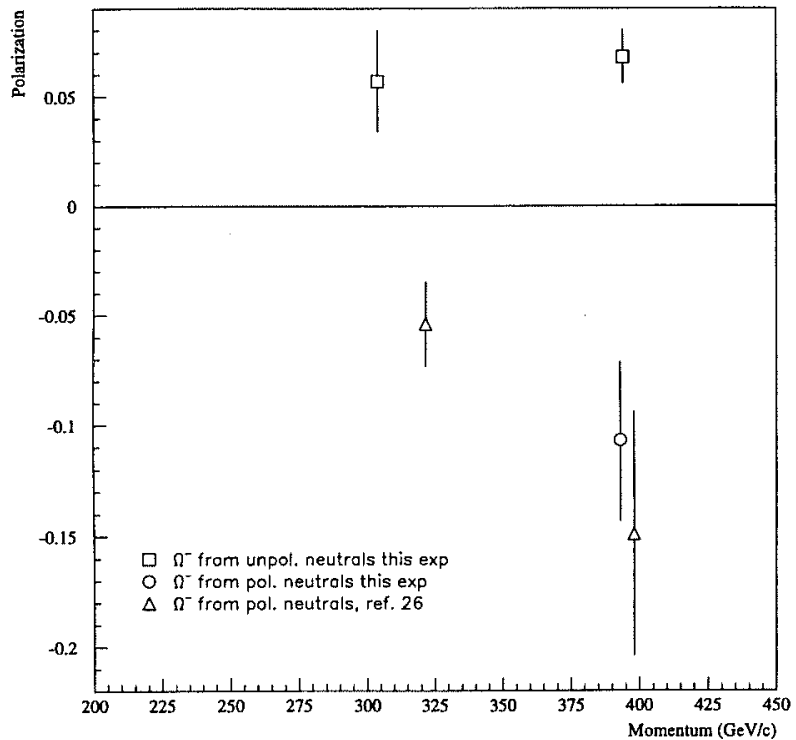

Figure 6.2: Comparision of $\Omega^{-}$polarization results. larization, $d=0$. To simplify the calculations, any differences between $\epsilon$ and $\epsilon^{\prime}$ and $\delta$ and $\delta^{\prime}$ are neglected, and we set $\delta \approx \epsilon$. In the development of their model, DeGrand and Miettinen [29] do not make a distinction between $\delta$ and $\delta^{\prime}$ or $\epsilon$ and $\epsilon^{\prime}$. In addition, they also assume $\delta \approx \epsilon$. In making a fit to experimental data, Kroll finds that $\delta$ and $\epsilon$ are not approximately equal, and that the primed and unprimed parameters are not similar [31]. In addition, it should be noted that since hyperon polarization is momentum dependent, these parameters are also momentum dependent. Differences of $10 \%$ between the values of $\delta$ and $\epsilon$ do not have a significant effect on any of the $\Xi^{-}$predictions. For the $\Omega^{-}$predictions, the difference between $\delta$ and $\epsilon$ is significant. Table 1.3 shows that for $\Omega^{-}$production from $\Lambda^{0} \mathrm{~s}$ and $\Xi^{0} \mathrm{~s}$, there are terms involving $\delta-\epsilon$. For $\epsilon$ fixed at a value of 0.1 , and $\delta$ varied between $0.9 \epsilon$ and $1.1 \epsilon$, the predicted polarization for $\Omega^{-}$s produced from $\Lambda^{0}$ s varies from -0.008 to +0.008 . The prediction for production from $\Xi^{0} \mathrm{~s}$ varies from -0.013 to +0.003 . Note that in both cases the magnitude of the predicted polarization is small.

The values used for the spin flip parameters are: $\tau_{1}=0.2$ and $\tau_{2}=0.4$. This extension to the model has a significant effect on several of the predictions. The values used for $\tau_{1}$ and $\tau_{2}$ come from experimental data [21], and the predictions are not sensitive to small variations in the values used for these parameters. The predicted polarizations for the different components of the neutral beam are given in Table 6.1. In this model, $-\epsilon$ is the prediction for the polarization of $\Lambda^{0}$ produced from protons, and a value of $\epsilon=0.1$ is used. 


\begin{tabular}{c|c|c|c} 
Interaction & $\begin{array}{c}\text { Predicted } \\
\text { Polarization }\end{array}$ & $\begin{array}{c}\text { Value for } \\
\epsilon=0.1\end{array}$ & $\begin{array}{c}\text { Value with no } \\
\text { spin flip }\end{array}$ \\
\hline$n \rightarrow \Xi^{-}$ & $-\epsilon$ & -0.1 & -0.1 \\
\hline$\Lambda^{0} \rightarrow \Xi^{-}$ & $-0.08 \epsilon$ & -0.009 & -0.017 \\
\hline$\Xi^{0} \rightarrow \Xi^{-}$ & $+0.8 \epsilon$ & +0.083 & +0.1 \\
\hline \hline$n \rightarrow \Omega^{-}$ & 0 & 0 & 0 \\
\hline$\Lambda^{0} \rightarrow \Omega^{-}$ & $\frac{5}{6}(\epsilon-\delta)$ & $-0.008-+0.008$ & \\
\hline$\Xi^{0} \rightarrow \Omega^{-}$ & $\frac{10(\delta-\epsilon)-0.8 \epsilon}{17.6}$ & $-0.013-+0.003$ &
\end{tabular}

Table 6.1: Predicted polarizations for production from an unpolarized neutral beam. See the text for details about the $\Omega^{-}$predictions.

The composition of our neutral beam and the cross sections for $\Xi^{-}$and $\Omega^{-}$production have not been measured, so combining these predictions is difficult. An attempt at estimating the sources of the final state $\Xi-s$ and $\Omega^{-} s$ has been made based on measurements of neutron, $\Lambda^{0}$ and $\Xi^{0}$ production cross sections [25]. Diehl assumed that the cross sections for $\Xi^{-}$and $\Omega^{-}$production by the components of the neutral beam were similar to other process with similar quark exchanges. This calculation predicted that $\Xi^{-} s$ were mostly produced by $\Lambda^{0} s$ followed by $\Xi^{0} s$, and that $\Omega^{-} s$ were produced by $\Xi^{0} \mathrm{~s}$ followed by $\Lambda^{0} \mathrm{~s}$, with neutron production playing a small role in both cases.

For the $\Xi^{-}$, the predicted polarization is small for $\Lambda^{0}$ production which is thought to be the dominant production mechanism. The predictions for neutron and $\Xi^{0}$ production are larger and have similar magnitudes, but opposite signs. With the estimates discussed above for the contributions of the various components of the neutral beam, a small negative polarization could be expected. Zero final state polarization is also possible with an appropriate combination of contributions from the various components of the neutral beam. Experimentally, no final state polarization is observed.

For $\Omega^{-}$production, the magnitude of the predicted polarization is small for all of the production mechanisms, and the signs of the polarization depends on the parameters in the models. The measured values of $+0.044 \pm 0.008$ and $+0.036 \pm 0.015$, are larger than all of the predictions of the model.

\begin{tabular}{c|c|c|c} 
Interaction & $\begin{array}{c}\text { Predicted } \\
\text { Polarization }\end{array}$ & $\begin{array}{c}\text { Incident Beam } \\
\text { Polarization }\end{array}$ & Predicted Value \\
\hline$n \rightarrow \Xi^{-}$ & $\frac{-1.6 d-10.8 \epsilon}{8 d \epsilon+10.8}$ & $\mathrm{~d}=-\frac{40}{42} \epsilon \approx-\epsilon$ & $-0.079--0.091$ \\
\hline$\Lambda^{0} \rightarrow \Xi^{-}$ & $\frac{d-0.6 \epsilon}{2 d \epsilon+6.8}$ & $\mathrm{~d}=-\epsilon$ & $-0.022--0.026$ \\
\hline$\Xi^{0} \rightarrow \Xi^{-}$ & $\frac{2 d+3.8 \epsilon}{4 d \epsilon+4.6}$ & $\mathrm{~d}=-\epsilon$ & $+0.033-+0.045$ \\
\hline \hline$n \rightarrow \Omega^{-}$ & 0 & 0 & 0 \\
\hline$\Lambda^{0} \rightarrow \Omega^{-}$ & $\frac{5}{6} \frac{0.8 d+1.2(\epsilon-\delta)}{1.2}$ & $\mathrm{~d}=-\epsilon$ & $-0.047--0.055$ \\
\hline$\Xi^{0} \rightarrow \Omega^{-}$ & $\frac{10(d-\epsilon+\delta)+0.8(5 \delta-6 \epsilon)}{2 d(5 \delta-4 \epsilon)+17.6}$ & $\mathrm{~d}=-\epsilon$ & $-0.054--0.069$
\end{tabular}

Table 6.2: Predicted polarizations for production from a polarized neutral beam. The range of predicted values show the effect of $a \pm 10 \%$ difference between the values of $\delta$ and $\epsilon$.

The predictions of this model for a polarized incident beam are given in Table 6.2. The range of predicted values show the effect of a $\pm 10 \%$ difference in the values of $\delta$ and $\epsilon$. The effect of neglecting the spin flip contributions is to increase the magnitude of the predicted $\Omega^{-}$polarizations by $2 \%-3 \%$. The values used for the incident beam polarization are based on the DeGrand and Miettinen model predictions for the production of the neutral beam components from protons.

For the $\Xi^{-}$, the largest predicted polarization is for production from neutrons, however, this interaction is not expected to contribute much to the final sample. The predictions for $\Lambda^{0}$ and $\Xi^{0}$ production have opposite signs, but similar magnitudes. The prediction for $\Lambda^{0}$ production, which is thought to be the dominant production mode, does have the same sign as the observed polarization, however, both of these predictions are much smaller in magnitude than the observed polarization of $-0.118 \pm 0.008$.

For the $\Omega^{-} \mathrm{s}$, no polarization is predicted for production from neutrons. The predicted polarization for $\Lambda^{0}$ and $\Xi^{0}$ production are similar in sign and magnitude $(-0.05$ to -0.07 ), and both predictions are consistent with the observed value of $-0.069 \pm 0.023$. 
Of the four cases studied in this experiment (production of $\Xi^{-} s$ and $\Omega^{-} s$ from polarized and unpolarized neutral beams), the model of DeGrand and Miettinen is successful only in the case of $\Omega^{-}$production by a polarized neutral beam. For $\Xi^{-}$production from a polarized neutral beam and $\Omega^{-}$production from an unpolarized neutral beam, the magnitudes of the observed polarizations are larger than the predictions. For $\Xi^{-}$production from an unpolarized neutral beam, no polarization is observed. In this case, the dominant contribution is thought to be $\Lambda^{0}$ production, which is predicted to be small. However, the polarization prediction for $\Xi^{0}$ production is large, so evaluating the success of the DeGrand and Miettinen model in this case is difficult.

\subsubsection{Other possible explanations}

In the DeGrand and Miettinen model, all of the quarks are treated in the same manner. However, it is certainly plausible to expect that the polarization of the quarks produced from the sea depends on the type of quark produced since the $s$ quark is heavier than the $u$ and $d$ quarks. It is interesting to note that all of the $\Omega^{-}$production reactions have a change in strangeness between the initial and final states, and that we observe polarization in our sample of $\Omega^{-}$produced from the unpolarized neutral beam. However, for the $\Xi^{-} \mathrm{s}$, production from a $\Xi^{0}$ is a $\Delta S=0$ interaction, and no polarization is observed in the unpolarized neutral beam production mode.

Results from a recent experiment could also support this idea [53]. In this experiment, a beam of $\Sigma^{-}$hyperons was used to produce a sample of $\Lambda^{0} \mathrm{~s}, \bar{\Lambda}^{0} \mathrm{~s}, \Sigma^{+} \mathrm{s}$, and $\Xi^{-} \mathrm{s}$. The polarization of the $\Xi^{-} \mathrm{s}$ was found to be similar in both magnitude and sign to samples of $\Lambda^{0}$ s produced from protons. However, the polarization of the $\Lambda^{0} s$ and $\Sigma^{+} s$ was found to be small or zero, but definitely not as large as the polarization of the $\Xi^{-}$s. The $\Lambda^{0}$ and $\Sigma^{+}$production process had no change in strangeness, whereas $\Xi^{-}$ production did involve a change in the number of strange quarks.
All of the quark level models that attempt to explain the polarization phenomena are based on the simple baryon wave-functions given in Table 1.1. It is possible that the actual baryon wave functions are more complicated. If, in addition to spin angular momentum, the ground state baryon wave functions also contained orbital angular momentum, then a possible explanation of the observed polarizations is that the production process favors certain orbital angular momentum configurations. Then, since the final state must have a total spin of $\frac{1}{2}$ (or $\frac{3}{2}$ for the $\Omega^{-}$), there could be a preferred spin orientation for the quark or quarks from the sea that combine with the incident fragment to produce the observed final state.

\subsection{Summary}

To summarize, the polarization of $\Xi^{-} \mathrm{s}$ and $\Omega^{-}$produced by an unpolarized neutral beam has been has been measured for the first time. The $\Xi^{-} s$ were found to be unpolarized. The observed $\Omega^{-}$polarization was $+0.044 \pm 0.008$ at an average momentum of 394. $\mathrm{GeV} / \mathrm{c}$ and $+0.036 \pm 0.015$ at an average momentum of $305 . \mathrm{GeV} / \mathrm{c}$. These values are smaller than the theoretical predictions. The sign of this observed $\Omega^{-}$polarization is also opposite that predicted by one theoretical model. This rules out the picture that hyperon polarization is due to the conservation of angular momentum during the production of quark - anti-quark pairs from the sea.

The polarization of $\Xi^{-} s$ and $\Omega^{-}$s produced by a polarized neutral beam was also studied. The $\Xi^{-}$sample had a polarization of $-0.118 \pm 0.004$ at an average momentum of 393 . $\mathrm{GeV} / \mathrm{c}$. The $\Omega^{-}$sample had a polarization of $-0.069 \pm 0.023$ at an average momentum of $394 . \mathrm{GeV} / \mathrm{c}$. Both of these results agree with a previous measurement. The observed polarization of both particles have larger magnitudes than theoretical predictions.

None of the current models that attempt to explain the phenomena of hyperon 
polarization gives a satisfactory explanation of these results. The results for production from an unpolarized neutral beam could indicate that polarization may only appear in interactions where there is a change in strangeness between the initial and final state. These results would also not rule out the idea that the ground state baryon wave functions involve orbital angular momentum components in addition to spin angular momentum components.

This experiment has studied the polarization of hyperons produced from an unpolarized beam of neutral particles. This is one of the first instances where the polarization of hyperons produced from baryons other than protons has been studied. The results of this experiment have pointed out problems with two theoretical models, and it is hoped that these results will offer further insight into the origin of hyperon polarization.

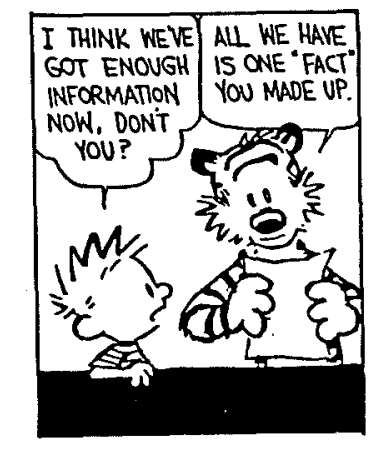

THAT'S PLENTY. BY THE TIME

WE ADD AN INTRODUCTION,

A FEW ILLUSTRATIONS, AN

A CONCLUSION, IT WILL
LOOK LKE A GRADUATE

LOOK LKE A GRADUNT
THESIS.

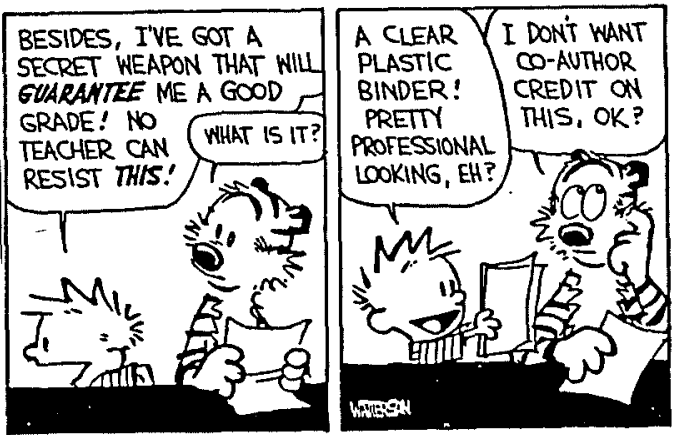

CALVIN AND HOBBES copyright Watterson. Dist. by UNIVERSAL PRESS SYNDICATE. Reprinted with permission. All rights reserved. 
as follows:

$$
\begin{aligned}
& \left\langle\Xi^{-} \uparrow|T| \Xi^{0} \uparrow\right\rangle=\frac{2}{3} B_{1} A_{11}+\frac{1}{3} B_{1} A_{10} \\
& \left\langle\Xi^{-} \uparrow|T| \Xi^{0} \downarrow\right\rangle=-\frac{1}{3} B_{\uparrow} A_{10} \\
& \left\langle\Xi^{-} \downarrow|T| \Xi^{0} \uparrow\right\rangle=-\frac{1}{3} B_{\downarrow} A_{10} \\
& \left\langle\Xi^{-} \downarrow|T| \Xi^{0} \downarrow\right\rangle=\frac{1}{3} B_{\downarrow} A_{10}+\frac{2}{3} B_{\uparrow} A_{1-1}
\end{aligned}
$$

\section{Calculation of Polarization}

\section{prediction for $\Xi^{0} \rightarrow \Xi^{-}$}

This appendix shows a sample calculation of a polarization prediction including the allowance for a spin flip before the recombination into the final state.

First, we start by writing the wave functions for the $\Xi^{0}$ and $\Xi^{-}$for both the spin up and spin down states. They are as follows:

$$
\begin{aligned}
& \Xi^{0} \uparrow: \sqrt{\frac{2}{3}} u \downarrow s s_{11}-\sqrt{\frac{1}{3}} u \uparrow s s_{10} \\
& \Xi^{0} \downarrow: \sqrt{\frac{1}{3}} u \downarrow s s_{10}-\sqrt{\frac{2}{3}} u \uparrow s s_{1-1} \\
& \Xi^{-} \uparrow: \sqrt{\frac{2}{3}} d \downarrow s s_{11}-\sqrt{\frac{1}{3}} d \uparrow s s_{10} \\
& \Xi^{-} \downarrow: \sqrt{\frac{1}{3}} d \downarrow s s_{10}-\sqrt{\frac{2}{3}} d \uparrow s s_{1-1}
\end{aligned}
$$

where $s s_{n m}$ is a shorthand for a pair of $s$ quarks in a spin state with total spin $n$ and spin z-component $m$. In the notation of DeGrand and Miettinen, this is a VVS process, meaning that the final state contains two valence quarks from the incident particle, two $s$ quarks in this case, and one quark from the sea, a $d$ quark here [30]. The first step, is to calculate the matrix element for each of the four possible interactions. They are

where $B_{\uparrow}$ and $B_{\downarrow}$ are the amplitudes due to spin up and spin down sea quarks, and $A_{n m}$ is the amplitude due to a valence quark pair in a state $s s_{n m}$. Next, we calculate the cross sections by taking the squares of these amplitudes, neglecting any incoherent cross-terms, and using the following parameterization of the valence and sea quark amplitudes:

$$
\begin{aligned}
\left|B_{\uparrow}\right|^{2} & =B(1-\epsilon) \\
\left|B_{\downarrow}\right|^{2} & =B(1+\epsilon) \\
\left|A_{1 m}\right|^{2} & =A(1+m \delta) \\
\left|A_{00}\right|^{2} & =A
\end{aligned}
$$

With this parameterization, the cross sections, $w_{b^{\prime} b}$, where $b$ is the spin of the produced particle and $b^{\prime}$ is the spin of the incident particle, are written as:

$$
\begin{aligned}
w_{\frac{1}{2} \frac{1}{2}} & =\frac{A B}{9}(5+4 \delta+3 \epsilon) \\
w_{\frac{1}{2}-\frac{1}{2}} & =\frac{A B}{9}(1-\epsilon) \\
w_{-\frac{1}{2} \frac{1}{2}} & =\frac{A B}{9}(1+\epsilon) \\
w_{-\frac{1}{2}-\frac{1}{2}} & =\frac{A B}{9}(5-4 \delta-3 \epsilon)
\end{aligned}
$$

Now, we can calculate $\mathcal{A}$, the asymmetry of this interaction. The asymmetry is the difference between the probability for a spin up final state minus the probability for a 
spin down final state over the total probability, and can be written as:

$$
\mathcal{A}=\frac{\sum_{b^{\prime}} \frac{1}{2}\left(w_{\frac{1}{2} b^{\prime}}-w_{-\frac{1}{2} b^{\prime}}\right)}{\sum_{b b^{\prime}}|b| w_{b b^{\prime}}}
$$

Doing this for the case with no polarization of the incident beam, we find:

$$
\mathcal{A}=\frac{\epsilon+2 \delta}{3}
$$

If this incident beam is polarized, with $x$ particles with spin up, and $(1-x)$ particles spin down, the cross sections calculated above need to be weighted accordingly. If this is done, the prediction for the asymmetry becomes:

$$
\mathcal{A}=\frac{2 d+\epsilon+2 \delta}{2 d(\delta+\epsilon)+3}
$$

where $d=2 x-1$ is the polarization if the incident beam.

This model has been extended by allowing for the possibility that the quark pair from the incident particle will undergo a spin flip before combining with the quark from the sea [21]. Allowing for this, the matrix elements calculated above become:

$$
\begin{aligned}
& \left\langle\Xi^{-} \uparrow|T| \Xi^{0} \uparrow\right\rangle=\frac{2}{3} B_{\downarrow} A_{11}+\frac{1}{3} B_{\uparrow} A_{10}-\frac{\sqrt{2}}{3} B_{\uparrow} A_{10}^{\tau}-\frac{\sqrt{2}}{3} B_{\downarrow} A_{11}^{\tau} \\
& \left\langle\Xi^{-} \uparrow|T| \Xi^{0} \downarrow\right\rangle=-\frac{1}{3} B_{\uparrow} A_{10}+\frac{\sqrt{2}}{3} B_{\downarrow} A_{11}^{\tau}+\frac{\sqrt{2}}{3} B_{\uparrow} A_{10}^{\tau} \\
& \left\langle\Xi^{-} \downarrow|T| \Xi^{0} \uparrow\right\rangle=-\frac{1}{3} B_{\downarrow} A_{10}+\frac{\sqrt{2}}{3} B_{\downarrow} A_{10}^{\tau}+\frac{\sqrt{2}}{3} B_{\uparrow} A_{1-1}^{\tau} \\
& \left\langle\Xi^{-} \downarrow|T| \Xi^{0} \downarrow\right\rangle=\frac{1}{3} B_{\downarrow} A_{10}+\frac{2}{3} B_{\uparrow} A_{1-1}-\frac{\sqrt{2}}{3} B_{\uparrow} A_{1-1}^{\tau}-\frac{\sqrt{2}}{3} B_{\downarrow} A_{10}^{\tau}
\end{aligned}
$$

where $A_{n m}^{\tau}$ is the amplitude due to a valence quark pair that has undergone a spin flip into a $s s_{n m}$ state.

With these new matrix elements, the cross sections are:

$$
w_{\frac{1}{2} \frac{1}{2}}=\frac{A B}{9}(5+4 \delta+3 \epsilon)+\frac{A B}{9} \tau_{2}(4+2 \delta)
$$

$$
\begin{aligned}
w_{\frac{1}{2}-\frac{1}{2}} & =\frac{A B}{9}(1-\epsilon)+\frac{A B}{9} \tau_{2}(4+2 \delta) \\
w_{-\frac{1}{2} \frac{1}{2}} & =\frac{A B}{9}(1+\epsilon)+\frac{A B}{9} \tau_{2}(4-2 \delta) \\
w_{-\frac{1}{2}-\frac{1}{2}} & =\frac{A B}{9}(5-4 \delta-3 \epsilon)+\frac{A B}{9} \tau_{2}(4-2 \delta)
\end{aligned}
$$

where the parameterization $\left|A_{m n}^{\tau}\right|^{2}=\tau_{2} A(1+m \delta)$ has been used. Here, $\tau_{2}$ is determined from the probability $\mathcal{P}$ of a single spin flip.

$$
\tau_{2}=\frac{2 \mathcal{P}(1-\mathcal{P})}{1-2 \mathcal{P}(1-\mathcal{P})}
$$

With these cross sections, the asymmetry can be calculated. Allowing for a polarized incident beam, the asymmetry is:

$$
\mathcal{A}=\frac{2 d+\epsilon+2 \delta\left(1+\tau_{2}\right)}{2 d(\delta+\epsilon)+3+4 \tau_{2}}
$$

As a consistency check, we see that if we set $\tau_{2}=0$, we get the expression in Equation A.19, and if we set $\tau_{2}=0$ and $d=0$, we get the result expressed in Equation A.18. 


\section{Appendix B}

\section{Hybrid Monte Carlo Topological}

\section{Selection Criteria}

A number of topological selection criteria were imposed on the real and fake events used in the hybrid monte carlo polarization analysis. Some of these selection criteria were dictated by the spectrometer. Any fake event wire hits in the MWPCs that fell outside the active region of a chamber were neglected. The fake events had to satisfy the $(C 11)_{\pi} \cdot(C 12)_{\text {proton }}$ part of the trigger.

Additional requirements involving the hits used on the various particle tracks and the separation of these track were imposed. Different criteria were used on the $\Omega^{-}$and $\Xi^{-}$samples. The small size of the $\Omega^{-}$sample dictated that the required hit and track separation requirements not reduce that sample size significantly, whereas the large $\Xi^{-}$ sample allowed more extensive cuts. The required hits are listed in Table B.1. These requirements were developed through extensive systematic studies. Similar systematic studies were performed to test the track separation cuts listed in Table B.2. The main purpose of these selection criteria was to correct for the known limitations of the reconstruction process.

\begin{tabular}{l|c|c|c|c|c} 
Chamber & view & Particle & Proton & $\pi$ & $\pi / \boldsymbol{L}$ \\
\hline \multirow{3}{*}{ C6 } & $\mathrm{X}$ & $\Xi^{-}$ & $\mathrm{X}$ & $\mathrm{X}$ & $\mathrm{X}$ \\
\cline { 3 - 6 } & & $\Omega^{-}$ & $\mathrm{X}$ & $\mathrm{X}$ & $\mathrm{X}$ \\
\cline { 2 - 6 } & $\mathrm{y}$ & $\Xi^{-}$ & $\mathrm{X}$ & $\mathrm{X}$ & $\mathrm{X}$ \\
\cline { 3 - 6 } & & $\Omega^{-}$ & & & \\
\hline \multirow{3}{*}{ C9 } & $\mathrm{X}$ & $\Xi^{-}$ & $\mathrm{X}$ & $\mathrm{X}$ & $\mathrm{X}$ \\
\cline { 3 - 6 } & & $\Omega^{-}$ & $\mathrm{X}$ & $\mathrm{X}$ & \\
\cline { 2 - 6 } & $\mathrm{y}$ & $\Xi^{-}$ & $\mathrm{X}$ & $\mathrm{X}$ & $\mathrm{X}$ \\
\cline { 3 - 6 } & & $\Omega^{-}$ & & & \\
\hline \multirow{3}{*}{ C10 } & $\mathrm{X}$ & $\Xi^{-}$ & & $\mathrm{X}$ & \\
\cline { 3 - 6 } & & $\Omega^{-}$ & & & \\
\cline { 2 - 6 } & $\mathrm{y}$ & $\Xi^{-}$ & $\mathrm{X}$ & $\mathrm{X}$ & $\mathrm{X}$ \\
\cline { 3 - 6 } & & $\Omega^{-}$ & $\mathrm{X}$ & $\mathrm{X}$ & $\mathrm{X}$
\end{tabular}

Table B.1: Summary of hits required in the hybrid Monte Carlo polarization analysis.

\begin{tabular}{l|c|c|c|c|c} 
Chamber & view & particle & $P-\pi$ & $\pi-\pi / S$ & $P-\pi / S$ \\
\hline \multirow{4}{*}{ C6 } & $\mathrm{x}$ & $\Xi^{-}$ & 3 & 2 & 2 \\
\cline { 3 - 6 } & & $\Omega^{-}$ & 2 & 1 & 1 \\
\cline { 2 - 6 } & $\mathrm{y}$ & $\Xi^{-}$ & 2 & 3 & 4 \\
\cline { 3 - 6 } & & $\Omega^{-}$ & 1 & 1 & 1 \\
\hline \multirow{4}{*}{ C9 } & $\Xi^{-}$ & 4 & 2 & 2 \\
\cline { 3 - 6 } & & $\Omega^{-}$ & 3 & 1 & 1 \\
\cline { 2 - 6 } & $\mathrm{y}$ & $\Xi^{-}$ & 3 & 2 & 3 \\
\cline { 3 - 6 } & & $\Omega^{-}$ & 3 & 1 & 3 \\
\hline \multirow{4}{*}{ C10 } & $\mathrm{x}$ & $\Xi^{-}$ & 1 & 3 & 1 \\
\cline { 3 - 6 } & & $\Omega^{-}$ & 1 & 1 & 1 \\
\cline { 2 - 6 } & $\mathrm{y}$ & $\Xi^{-}$ & 1 & 1 & 3 \\
\cline { 3 - 6 } & & $\Omega^{-}$ & 1 & 1 & 1 \\
\hline \multirow{3}{*}{ C11 } & $\mathrm{x}$ & $\Xi^{-}$ & 0 & 0 & 0 \\
\cline { 3 - 6 } & & $\Omega^{-}$ & 0 & 0 & 0 \\
\cline { 2 - 6 } & $\mathrm{y}$ & $\Xi^{-}$ & 1 & 1 & 1 \\
\cline { 3 - 6 } & & $\Omega^{-}$ & 1 & 1 & 1 \\
\hline
\end{tabular}

Table B.2: Summary of track separations required in the hybrid Monte Carlo polarization analysis. The separations are expressed in terms chamber wire spacings, which is $2 \mathrm{~mm}$ for all the chambers listed in the table. 


\section{References}

[1] R. M. Woods and B. J. Spence, Phys. Rev. 4, 669, (1934).

[2] R. M. Woods, Jr., Ph.D. Thesis, "Improvements in Beta Spectroscopy and the Decay of Samarium-153, Dysprosium-165, and Cesium-134", University of Michigan, (1963), unpublished.

[3] G. Bunce et al., Phys. Rev. Lett. 36, 1113, (1976).

[4] R. O. Polvado et al., Phys. Rev. Lett. 42, 1325, (1979).

[5] K. Heller et al., Phys. Rev. Lett. 41, 607, (1978).

[6] C. Ankenbrandt et al., Phys. Rev. Lett. 51, 863, (1983).

[7] L. Deck et al., Phys. Rev. D 28, 1, (1983).

[8] C. Dukes et al., Phys. Lett. 193B, 135, (1987).

[9] A. Morelos et al., Phys. Rev. Lett. 71, 2172, (1993).

[10] R. Rameika et al., Phys. Rev. D 33, 3172, (1986).

[11] K. Heller et al., Phys. Rev. Lett. 51, 2025, (1983).

[12] P. M. Ho et al., Phys. Rev. Lett. 65, 1713, (1990).

[13] K. B. Luk et al., Phys. Rev, Lett. 70, 900, (1993).
[14] J. Bensinger et al., Phys. Rev. Lett. 50, 313, (1983).

[15] B. Edelman et al., Phys. Rev. Lett. 40, 491, (1978).

[16] S. U. Chung et al., Phys. Rev. D 11, 1010, (1975).

[17] G. T. Jones et al., Z. Phys. C 28, 23, (1985).

[18] K. Abe et al., Phys. Rev. D 29, 1877, (1984).

[19] B. E. Bonner et al., Phys. Rev. Lett. 58, 447, (1987).

[20] B. E. Bonner et al., Phys. Rev. D 38, 729, (1988).

[21] B. E. Bonner et al., Phys. Rev. Lett., 62, 1591, (1989).

[22] T. Henkes et al., Phys. Lett. 283B, 155, (1992).

[23] B. Lundberg et al., Phys. Rev. D 40, 3557, (1989).

[24] J. Duryea et al., Phys. Rev. Lett. 67, 1193, (1991).

[25] H. T. Diehl, Ph.D. thesis, "Omega Minus Polarization and Magnetic Moment", Rutgers University, (1990), unpublished.

[26] H. T. Diehl et al., Phys. Rev. Lett. 67, 804, (1991).

[27] G. L. Kane et al., Phys. Rev. Lett. 41, 1689, (1978).

[28] F. Halzen and A. D. Martin, "Quarks and Leptons", p. 171, John Wiley \& Sons, (1984).

[29] T. A. DeGrand and H. I. Miettinen, Phys. Rev. D 24, 2419, (1981).

[30] T. A. DeGrand et al., Phys. Rev. D 32, 2445, (1985). 
[31] P. Kroll, in High Energy Spin Physics Eighth International Symposium, edited by K. Heller, AIP Conference Proceedings No. 187 (American Institute of Physics, New York, 1989), p. 48

[32] M. W. Sullivan et al., Phys. Rev. D 36, 674, (1987).

[33] B. Andersson, G. Gustafson, and G. Ingelman, Phys. Lett. 85B, 417, (1979).

[34] J. Szwed, Phys. Lett. 105B, 403, (1981).

[35] W. G. D. Dharmaratna and G. R. Goldstein, Phys. Rev. D 41, 1731, (1990).

[36] R. Barni et al., Phys. Lett. 296B, 251, (1992).

[37] J. Soffer and N. A. Törnqvist, Phys. Rev. Lett. 68, 907, (1992).

[38] H. Haggerty et al., NIM 115, 157, (1974).

[39] S. Hansen et al., IEEE Trans. Nuc. Sci. NS-34, 1003, (1987)

[40] Particle Data Group, Phys. Rev. D 50, 1173, (1994).

[41] T. D. Lee et al., Phys. Rev. 106, 1367, (1957).

[42] T. D. Lee and C. N. Yang, Phys. Rev. 108, 1645, (1957).

[43] O. E. Overseth and S. Pakvasa, Phys. Rev. 184, 1663, (1969).

[44] M. Deutschmann et al., Phys. Lett. 73B, 98, (1978).

[45] M. Baubillier et al., Phys. Lett. 78B, 342, (1978).

[46] K. B. Luk et al., Phys. Rev. D 38, 19, (1988).

[47] G. M. Guglielmo, Ph. D. thesis, "Measurement of the Decay Asymmetries of the Omega-Minus Baryon”, University of Minnesota, (1994), unpublished.
[48] J. Finjord, Phys. Lett. 76B, 116, (1978).

[49] J. Finjord and M. K. Gaillard, Phys. Rev. D 22, 778, (1980).

[50] H. Galic, D. Tadic, and J. Trampetic, Phys. Lett. 89B, 249, (1980).

[51] G. Bunce, NIM 172, 553, (1980).

[52] N. B. Wallace, Ph. D. thesis, "A Precision Measurement of the Magnetic Moment of the Omega Minus Hyperon", University of Minnesota, (1995), unpublished.

[53] M. I. Adamovich et al., CERN-PPE/94-86, (1994). 